\title{
Role of the Different Domains of PSD-95 in Basal Synaptic Transmission
}

\author{
Dissertation \\ for the award of the degree \\ Doctor rerum naturalium (Dr. rer. nat.) \\ Division of Mathematics and Natural Sciences \\ of the Georg-August-Universität Göttingen
}

submitted by

Stéphanie A.D. Bonnet

from Marseilles, France 
Göttingen, August 2011 


\section{Thesis committee members}

\section{Dr. Dr. Oliver Schlüter (reviewer)}

Molecular neurobiology, European Neuroscience Institute Göttingen

\section{Prof. Dr. Reinhard Jahn (reviewer)}

Dept. of Neurobiology, Max Planck Institute for Biophysical Chemistry, Göttingen

\section{Prof. Dr. Jürgen Klingauf}

Dept. of Cellular Biophysics, Institute of Medical Physics and Biophysics

\section{Extended committee members}

\section{Prof. Dr. M.Hörner}

Dept. of Cell Biology, Johann-Friedrich-Blumenbach-Institute for Zoology and Anthropology, Göttingen

\section{C.Dean, PhD}

Trans-synaptic Signaling, European Neuroscience Institute Göttingen

\section{Prof. Dr. T.Dresbach}

Dept. of Anatomy and Embryology, Center of Anatomy, Göttingen 
Date of oral examination: September $23^{\text {rd }}, 2011$

I hereby declare that I prepared the PhD thesis "Role of the different domains of PSD-95 in basal synaptic transmission" on my own and with no other sources and aids than quoted. 
Stéphanie A.D Bonnet 


\begin{abstract}
Learning and memory has been postulated to rely on changes in synaptic transmission strength. At excitatory synapses in the hippocampus CA1 region, synaptic strength is mainly regulated by AMPA receptor function at the postsynaptic density. PSD-95, a member of DLG-MAGUK family along with PSD-93, SAP97 and SAP102, is involved in the regulation of AMPAR function. DLGMAGUKs share a similar modular domain structure, composed of three copies of PDZ domain, an SH3 domain and a catalytically inactive GK domain. PSD-95 is the most abundant DLG-MAGUK at excitatory mature synapses and interacts with various cellular proteins.

To examine the requirement of specific PSD-95 domains and their role in the excitatory synaptic transmission regulation, I combined two approaches. On one hand, I made use of the molecular replacement approach by acutely knocking-down PSD-95 and expressing simultaneously a mutant form of PSD-95, in a spatiotemporally specific manner among an intact network of neurons in rat hippocampal slices. On the other hand, I took advantage of the PSD-95 knockout mouse line, to express mutants of PSD-95 in a PSD-95 free background. To assess which domain of PSD-95 is important for the protein to mediate its effect on basal synaptic transmission, I evaluated if specific form of truncated PSD-95 could rescue the deficiency caused by the absence of endogenous PSD-95.

With this study I could show that PSD-95 lacking its PDZ3, SH3 and GK domains could not mediate proper basal synaptic strength in the absence of endogenous PSD-95. Moreover, the PDZ3 or SH3 domain seems dispensable for the protein to be functional. Finally, to be functional PSD-95 requires its GK domain. However, this seems dependent on SAP102, another DLG-MAGUK
\end{abstract}


regulating synaptic transmission in immature synapses. The requirement of the GK domain appears dependant on the maturational state of the synapse with a strongest effect on more immature synapses. In conclusion, my data demonstrate the importance of the N-terminal PDZ12 domains acting in concert with the GK domain and a permissive role of the SH3 and PDZ3 domains in regulating the strength of AMPAR function.

Keywords: PSD-95, GK domain, AMPAR function, basal synaptic transmission. 


\section{Acknowledgments}

I would like to thank Dr. Dr. Oliver Schlüter for giving me the opportunity to do my PhD in his lab and for the supervision of my thesis. I am very grateful for the scientific training I have gained during the course of my Ph.D and tried to learn from his unlimited optimism and creativity.

My thankfulness goes to Prof. Dr. R. Jahn and Prof. Dr. J. Klingauf for their scientific support and helpful discussions as members of my thesis committee.

I am very thankful to the additional committee members, Prof. Dr. M.Hörner, Dr. C.Dean, Prof. Dr. T. Dresbach for taking their time to evaluate my work. 
Dr. Dr. O. Schlüter assembled an extraordinary team that worked in a professional and at the same time supportive and helpful atmosphere.

For that, I would like to thank the past and present members with whom I worked. Juliane, Martina, Yanling, Sandra, Ania, Derya, Huang, Tanmoy and Plinio. Not only working together, we became friends! Thank you for helping me having such a great time in the lab! I have to give my special thanks to Juliane, Ania and Derya for their amazing personal support!

Although I truly deeply hate Göttingen (for the weather), I have to give it the benefit of its extraordinary international environment. Here I met such beautiful people!

My strudel family was always there to support me in every possible way! So much time spend together and so much good memories! Will never forget the beautiful dinners at Matthieu's place, the amazing DJing by Alwaleed, the patriarchal despotism fight by Ahmed, the awesome barbecues at Jonas' place, and the happy talking by Andrea! I have to give un énorme merci to two exceptional girls I met by coming to Germany: Sanaz and Derya. You girls know me very well, and I'm very grateful for that! You added a lot of sunshine and balance to my time here.

Il y a ceux que j'ai quittés en prenant la décision de venir poursuivre mes études en Allemagne. La distance en catalyseur, et les meilleures sont restées ! Emilie, tu remercieras le Neuf de ma part, sans lui on n'en serait pas là aujourd'hui ! Une amitié à toute épreuve. Merci pour ton soutien hebdomadaire et nos discussions enjouées !

Je tiens aussi à remercier Lily, qui depuis janvier égaille mes journées et particulièrement ces longs mois d'écriture !! Aurél, merci à toi pour toutes ces années d'amitiés, quelle personne entière tu fais et je t'admire pour ça !

Et puis il y a ma famille, quelle famille d'ailleurs ! Je tiens à remercier chacun de mes couz, certaines personnes nous embellissent la vie. Les larmes me viennent en écrivant ... mais sinon ce ne serait pas moi ! Merci pour tout ce que vous m'avez apporté et allez continuer de m'apporter, car oui, on va en passer du temps ensemble!

Et puis il y a tous ceux de la Yaute, quel peuple merveilleux ! ... en quelques mots vous me faites rêver les copains ! 
Maman, Papa, sans vous rien de tout ça ne se serait passé. Merci pour votre support inconditionnel, ça n'a pas toujours été facile et pourtant vous avez toujours été présents. Sans jamais oser vous le dire, je vous admire chacun pour ce que vous êtes.

\section{Contents}

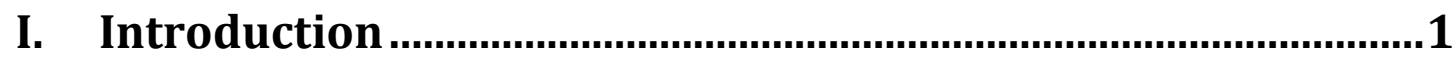

\section{I.A. The hippocampus as a model system to study basal synaptic} transmission

I.B. Glutamatergic Excitatory Synapses............................................. 7

I.B.1. The Post Synaptic Density.........................................................................

I.C. Ionotropic Glutamate Receptors.................................................. 8

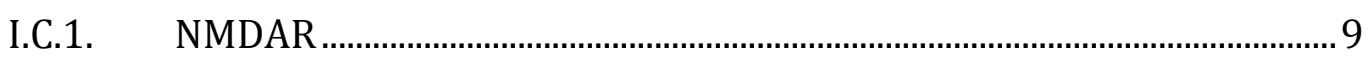

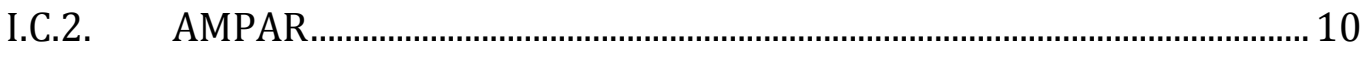

I.C.2.a. Structure and Composition of AMPARs .................................................. 11 
I.C.2.b. AMPAR biosynthesis and trafficking ………………………….......... 13

I.C.2.c. AMPAR modulating proteins …………………………………........ 15

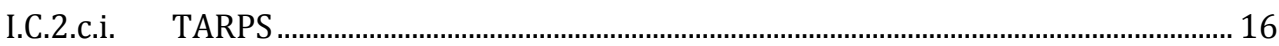

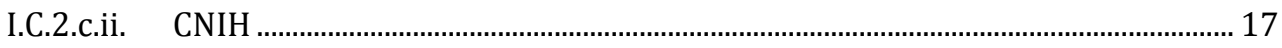

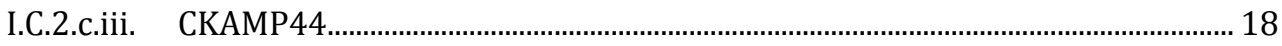

I.D. DLG-MAGUK................................................................................19

I.D.1. Structural organization of the DLG-MAGUKs ........................................ 20

I.D.2. Distributions of the DLG-MAGUKs ….................................................. 22

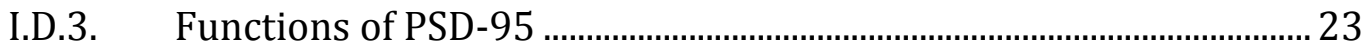

I.D.3.a. Role of PSD-95 in synaptic maturation ................................................... 23

I.D.3.b. Role of PSD-95 in synaptic plasticity ………………………………..... 24

I.D.3.c. Role of PSD-95 in synaptic AMPAR function ....................................... 24

I.D.3.c.i. Overexpression of PSD-95 ................................................................................... 24

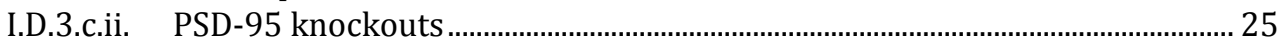

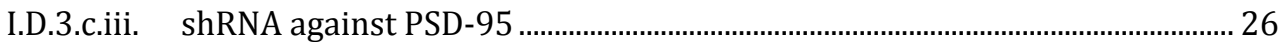

I.D.3.d. Roles of PSD-95 domains in basal synaptic transmission ............... 27

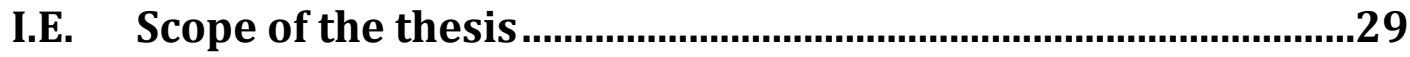

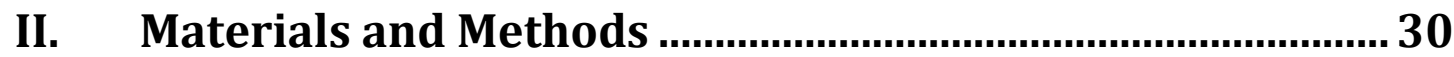

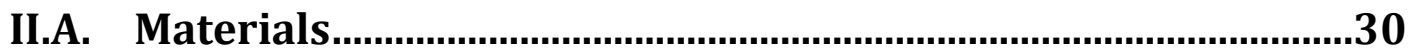

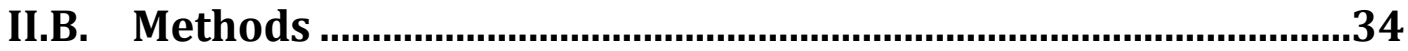

II.B.1. Molecular biology and biochemistry ............................................ 34

II.B.1.a. Electroporation of bacteria …………................................................. 34

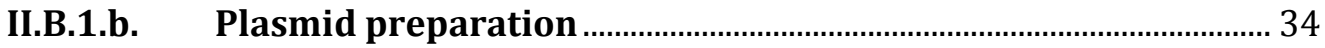

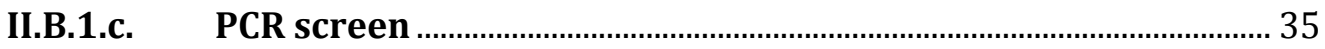

II.B.1.d. Ligation of DNA fragments …………………………………........... 36

II.B.1.e. Polymerase Chain Reaction (PCR)..................................................... 36

II.B.1.f. DNA gel electrophoresis........................................................................ 37

II.B.1.g. Extraction of DNA fragments from agarose gels............................ 38

II.B.1.h. Purification of DNA fragments after PCR..................................... 38

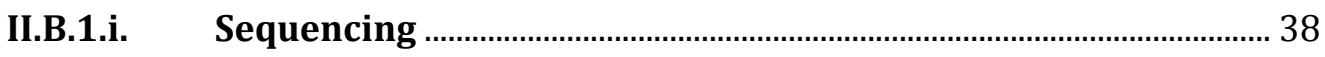

II.B.1.j. Generation of the mutants ............................................................ 39 
II.B.1.k. Pure knock down of endogenous PSD-95.

II.B.1.l. Molecular replacement of endogenous PSD-95 by its mutant: 39

II.B.1.l.i. The Molecular replacement technique .................................................................. 39

II.B.1.l.ii. Overexpression vectors...................................................................................... 43

II.B.1.m. List of primers ………..................................................................... 43

II.B.1.n. SDS-PAGE \& Western Blot ........................................................................ 45

II.B.1.o. Immunodetection ……………………................................................. 46

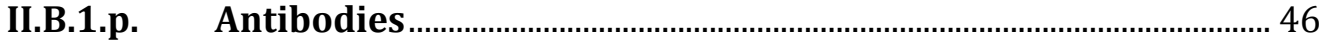

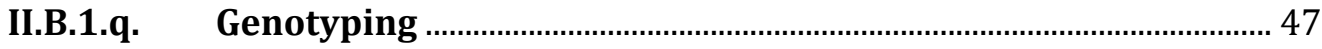

II.C. Cell culture techniques...........................................................48

II.C.1.a. Large scale lentivirus preparation........................................................ 49

II.C.1.b. Transfection...................................................................................... 49

II.C.1.c. Hippocampal dissociated culture …................................................... 50

II.C.1.d. Infection of dissociated culture …….................................................. 51

II.C.1.e. Harvesting dissociated culture for western blotting ................... 51

II.C.1.f. Hippocampal organotypic slices ..................................................... 51

II.C.1.g. Virus injection into CA1 hippocampal organotypic slice............ 52

II.C.1.h. Feeding schedule for organotypic slice ........................................... 53

II.D. Electrophysiology ..................................................................53

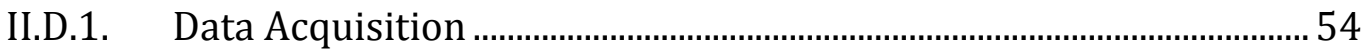

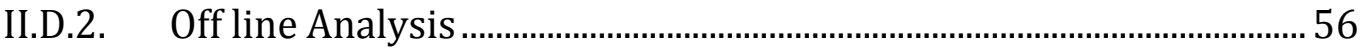

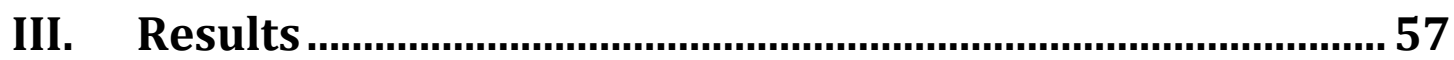

III.A. Molecular replacement studies in rat hippocampal organotypic slice cultures .....................................................59

III.A.1. shRNA mediated knock-down of PSD-95 in hippocampal dissociated culture .59

III.A.2. Effect of PSD-95 knock-down on basal synaptic transmission....... 60

III.A.3. Molecular replacement by full-length PSD-95 ……........................... 62

III.A.4. Molecular replacement by PSD-95 expressing PDZ12 domains .... 64

III.A.5. Molecular replacement by PSD-95 lacking its PDZ3 domain ......... 67 
III.A.6. Molecular replacement by PSD-95 lacking its SH3 domain 69

III.A.7. Molecular replacement by PSD-95 lacking its GK domain. 71

III.A.8. Molecular replacement using a mutant form of PSD-95 expressing PDZ12 and GK domains.

III.A.9. Overexpression of PSD-95 lacking its GK domain or containing only PDZ12-GK domains.

III.A.10. Molecular replacement by PSD-95 $\Delta$ GK and activity manipulation 84

III.B. Overexpression studies in PSD-95 KO mouse hippocampal organotypic slice cultures ......................................................86

III.B.1. Expression of PSD-95 PDZ12 in PSD-95 KO neurons........................... 86

III.B.2. Expression of full-length PSD-95 in PSD-95 KO neurons................... 88

III.B.3. Expression of PSD-95 lacking its SH3 domain in PSD-95 KO neurons 89

III.B.4. Expression of PSD-95 lacking its GK domain in PSD-95 KO neurons 90

III.B.5. Expression of PSD-95 PDZ12-GK in PSD-95 KO neurons. 91

\section{III.C. Molecular replacement studies in PSD-95 KO mouse} hippocampal organotypic slice cultures

III.C.1. SAP102 knockdown combined with PSD-95 $\Delta$ SH3 expression in PSD-95 KO neurons.

III.C.2. SAP102 knockdown combined with PSD-95 $\Delta$ GK expression in PSD-95 KO neurons

III.C.3. SAP102 knockdown combined with PSD-95 PDZ12-GK expression in PSD-95 KO neurons 97

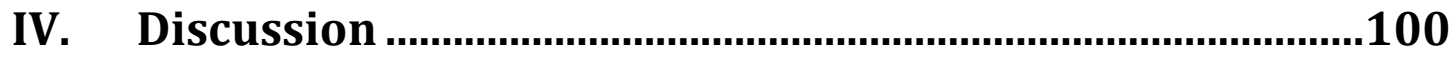

IV.A. Validation of the approaches ……..........................................100

IV.A.1. Domain deletion .............................................................................. 100 


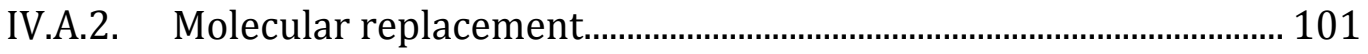

IV.A.3. Validation of the mutants ..................................................................... 102

IV.B. Domain requirement: ..........................................................102

IV.B.1. PDZ3 or SH3 domains are not required for PSD-95 to mediate basal synaptic transmission

IV.B.2. GK domain is required for PSD-95 to mediate basal synaptic transmission, when SAP102 does not compensate

IV.C. Differences in the maturational state of synapses between acutely knocked-down PSD-95 and germline removal of PSD-95.

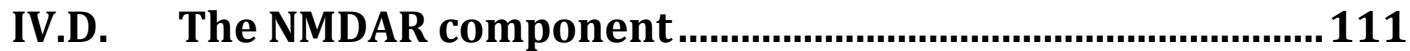

IV.E. Conclusion and outlook ........................................................112

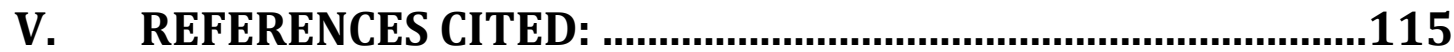

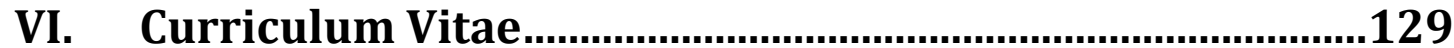

\section{List of figures}

Figure 1. Cartoon depicting the basic anatomy of the hippocampus.

Figure 2. Glutamatergic excitatory synapse visualized by electron microscopy. The presynaptic site is filled with neurotransmitter containing vesicles. The red arrow indicates the post-synaptic density, an electron dense area. (Adapted from (Kennedy, 2000). 8 
Figure 3. Illustration depicting a glutamatergic synapse.

Figure 4. Schematic illustration showing the topology of an AMPA receptor subunit..

Figure 5. AMPARs are exchanged between synaptic, extra-synaptic and intracellular compartments

Figure 6. Schematic diagram of PSD-95 domains showing the modular protein interaction motifs including three PDZ domains in tandem, an SH3 domain and a GK domain...... 20

Figure 7. Illustration of an SH3-GK intra- and inter-molecular interactions. 21

Figure 8. Simultaneous dual whole-cell patch-clamp recordings of hippocampal organotypic slice cultures.

Figure 9. Acute knockdown, using short hairpin RNA against PSD-95 (sh95) delivered by lentivirus. 60

Figure 10. Effects of PSD-95 knockdown in basal synaptic transmission. 62

Figure 11. Effects of PSD-95 molecular replacement in basal synaptic transmission. 64

Figure 12. Molecular replacement by wild type PSD-95 in rat hippocampal dissociated cultures assessed by western-blot.

Figure 13. Effects of PSD-95 PDZ12 molecular replacement on basal synaptic transmission. 66

Figure 14. Molecular replacement by PSD-95 PDZ12 in rat hippocampal dissociated cultures assessed by western-blot. 66

Figure 15. Effects of PSD-95 PDZ3 molecular replacement on basal synaptic transmission. 68

Figure 16. Molecular replacement by PSD-95 PDZ3 in rat hippocampal dissociated cultures assessed by western-blot.

Figure 17. Effects of PSD-95 SSH3 molecular replacement in basal synaptic transmission. 70

Figure 18. Molecular replacement by PSD-95 $\Delta$ SH3 in rat hippocampal dissociated cultures assessed by western-blot 71

Figure 19. Effects of PSD-95 GGK molecular replacement on basal synaptic transmission 72

Figure 20. Molecular replacement by PSD-95 $\Delta$ GK in rat hippocampal dissociated cultures assessed by western-blot.

Figure 21.Comparison of the different knockdown effects and expression levels of each truncated form of PSD-95 molecular replacement vector.. 73

Figure 22. Effects of PSD-95 PDZ12-GK molecular replacement on basal synaptic transmission. 77 Figure 23. Molecular replacement by PSD-95 PDZ12-GK in rat hippocampal dissociated cultures assessed by western-blot. 77

Figure 24. Effects of PSD-95 GGK overexpression on basal synaptic transmission. 79

Figure 25. Overexpression of PSD-95 GK in rat hippocampal dissociated cultures assessed by western-blot. 79

Figure 26. Effects of PSD-95 PDZ12-GK overexpression on basal synaptic transmission. 80

Figure 27. Overexpression of PSD-95 PDZ12 in rat hippocampal dissociated cultures assessed by western-blot. 80 
Figure 28. Variability of the results observed with PSD-95 $\Delta$ GK molecular replacement.

Figure 29. Effects of activity manipulations on PSD-95 $\Delta$ GK molecular replacement on basal

synatptic transmission.

Figure 30. Effects of PSD-95 PDZ12 overexpression on basal synaptic transmission in PSD-95 KO

mice. 87

Figure 31. Effects of PSD-95 overexpression on basal synaptic transmission in PSD-95 K0 mice.

Figure 32. Effects of PSD-95 SSH3 overexpression on basal synaptic transmission in PSD-95 K0

mice.

Figure 33. Effects of PSD-95 $\Delta$ GK overexpression on basal synaptic transmission in PSD-95 KO mice.

Figure 34. Effects of PSD-95 PDZ12-GK overexpression on basal synaptic transmission in PSD-95

KO mice. 92

Figure 35. Acute knockdown, using short hairpin RNA against SAP102 (sh102) delivered by lentivirus.

Figure 36. Effects of SAP102 knockdown combined with PSD-95 $\Delta$ SH3 overexpression on basal synaptic transmission in PSD-95 KO mice.

Figure 37. Effects of SAP102 knockdown combined with PSD-95 $\Delta$ GK overexpression on basal synaptic transmission in PSD-95 KO mouse.

Figure 38. Effects of SAP102 knockdown combined with PSD-95 PDZ12-GK overexpression on basal synaptic transmission in PSD-95 KO mice. 98 
et d'entendre au fond du gouffre

un léger souffle qui murmure que nous attend, comme un soleil impensable,

le Bonheur.

Boris Cyrulnik 


\section{Introduction}

The brain is the pillar of the human spirit. It is the center of cognitive functions such as learning, memory, language, consciousness and many other processes. Human behaviors and cognitive functions rely on accurate networking between single units of the brain, the neurons. Although the architecture of the brain circuits underlying basic conserved behaviors is encoded in our genetic material, a substantial part of who we are is based on our capacity to learn and remember new knowledge about the world. How does the brain acquire this new knowledge, and, more precisely, what are the molecular and cellular mechanisms underlying this information storage? The answer to the above questions lies in adaptive neuronal communication.

Neurons are highly organized cells. Morphologically and also functionally, a neuron consists of three compartments: the soma, the axon and the dendritic tree. From the soma, membrane extensions including a single axon and multiple arborized dendrites arise. Usually, neurons use trains of action potentials (AP) for coding and propagating patterns of information. The all-or-none property of action potential transmission prevents it from fading before it reaches the terminal of the axon, the synapse. An AP consists of rapid reversals of ionic charges flowing across the axonal plasma membrane, which are mediated by voltage-gated ions channels. This depolarization propagates all the way from the initial segment to the nerve terminal, allowing for the transmission of information from the cell soma to the more distal regions of the cell. At the end of the axon terminal, another neuron receives the information.

This information transmission between two neurons happens at connection points termed "synapses." Etymologically, the word "synapse" comes 
from Greek and originates from the fusion of the word syn, (together) with the word haptein, (attach). In the human brain, the $10^{11}$ neurons receive and make on average 10000 synaptic contacts per cell in a specific, organized and plastic manner, allowing the brain to maintain the behavioral stability of the organism over time. The synapse is a macromolecular complex making a connection between two neurons. There are two main types of synaptic junctions: the electrical and the chemical synapse. The main difference between these two types of neuronal synapses resides in the fact that chemical synapses are made of gap junction channels that physically bridge two neurons and allow charge transmission between these two, while chemical synapses connect two neurons via a synaptic cleft using neurotransmitters.

Around $99 \%$ of the synapses in the vertebrate central nervous system (CNS) are based on chemical communication. The junction has a specific orientation with a defined presynaptic process and postsynaptic process, separated by the synaptic cleft. In summary, the presynaptic bouton contains synaptic vesicles filled with neurotransmitters. Upon the arrival of an action potential, the presynaptic membrane depolarizes, which results in the voltage dependent calcium channels opening. This leads to a series of steps ending with the fusion of docked synaptic vesicles to the plasma membrane, which triggers the release of a packet (quantum) of transmitter substance. These neurotransmitters cross the narrow synaptic cleft $(20-25 \mathrm{~nm})$ and act on the postsynaptic branch juxtaposed to the presynaptic process. The postsynaptic part is able to respond to the neurotransmitter action, as it contains specific receptors and coupled machineries enabling neurons to transmit and regulate the information. Multiple depolarization events sum together and propagate to the cell body where, if the firing threshold is reached, a new action potential is generated in the proximity of the cell body at the axon hillock. From an operational point of view, a synapse converts a presynaptic electrical signal into a chemical signal and then back into a postsynaptic electrical signal. 
Synapses can be excitatory, inhibitory or modulatory. In the vertebrate brain, glutamate is the major neurotransmitter of excitatory synapses, while inhibitory transmission is mainly mediated by GABA (gamma-amino butyric acid) and glycine. The modulatory transmission is based on the action of dopamine, serotonin and other neuromodulators. The information propagation from neuron to neuron depends on the combined effects of excitatory, inhibitory and modulatory transmissions. Excitatory transmission relies on temporary depolarization of the postsynaptic membrane, a so-called excitatory postsynaptic potential (EPSP). An EPSP is caused by the activation of receptors, such as ionotropic glutamate receptors located at the postsynaptic cell membrane. On the contrary, inhibitory postsynaptic potentials (IPSP) regulate the action of the EPSC. EPSCs and IPSCs have additive effects. Larger EPSPs result in greater membrane depolarization, and thus increase the likelihood for the postsynaptic neuron to reach the threshold for firing an action potential, thus propagating the information. Finally, the summation of excitatory and inhibitory signals will fine tune neuronal excitability and thus neuronal transmission. This process is regulated by the number of excitatory versus inhibitory contacts received by a single neuron and their respective strengths.

Synapses that convey specific neurotransmitters differ in their composition and structure. Early on it has been discovered that synapses in the brain fall into two major categories: type I synapses, with asymmetrical densification of their pre and postsynaptic membranes, usually implicated in excitatory transmission; and type II synapses, with symmetrical densification mainly representing inhibitory synaptic actions. Excitatory and inhibitory synapses also differ in postsynaptic morphology. The majority of glutamatergic synapses are found on bulbous protrusions known as spines, whereas most GABAergic synapses are formed on the dendritic shaft, the soma or proximal axonal regions.

By using different neurotransmitters and neuromodulators, different types of receptors and different secondary messenger systems, a synapse exhibits 
considerable flexibility of its function. These features are crucial for allowing synapses to communicate between neurons, and thus elaborate complex circuits.

This complex networking and regulation of neuronal communications are under intensive investigation. Indeed, modifying the synaptic strength between neurons has been hypothesized to be the cellular correlate of learning and memory (Bliss and Lomo, 1973) (Wenthold et al., 2003) (Nicoll et al., 2006) (Malinow and Malenka, 2002) (Bredt and Nicoll, 2003). This process is referred to as synaptic plasticity. There are multiple forms of synaptic plasticity, which depend on their induction mode, expression site, the developmental stage and the type of synapses. One of the most-characterized plasticity processes is Long Term Potentiation (LTP) and its counterpart Long Term Depression (LTD) (Bliss and Lomo, 1973) (Martin et al., 2000) (Malenka and Nicoll, 1999) , which takes place at hippocampal CA3-CA1 synapses.

The homeostasis of the nervous system relies on proper communication between neurons, a process based on a harmonic operation between excitatory, inhibitory and modulatory transmissions. The dysfunction of any of these key players has been shown to mediate various neuropsychiatric disorders such as anxiety, epilepsy, psychosis and many others.

Thus, it becomes apparent that understanding the mechanisms of basic synaptic transmission is an important step towards the comprehension of brain circuit function in normal and pathological conditions.

\section{I.A. The hippocampus as a model system to study basal synaptic transmission}

As already mentioned, functional synaptic communication requires highly organized networks as well as flexibility. Indeed, wiring the brain involves the 
refinement of connections upon stimulation. This process is referred as synaptic plasticity and is thought to be the molecular basis underlying learning and memory. Since the 1950 's, the hippocampus has been recognized to play a fundamental role in episodic memory and learning, especially spatial learning (Penfield., 1955) (Kesner, Aug 2006) (Penfield., 1955, P. Andersen, 2007) (Kesner and Hopkins, 2006). Another reason making the hippocampus attractive is its highly laminated structure. Hence, it has a very distinctive and readily identifiable structure arranged in orderly layers.

The pyramidal cell layer has been divided into three regions designated as CA1, CA2, and CA3 (Cornu Amonnis or Ammon's horn) based on the size and morphology of the neurons. The regions above and below the pyramidal cell layer are divided into a number of strata (containing mainly inhibitory interneurons). The hippocampal formation contains the dentate gyrus (DG), composed of granular cell layers which connect to the CA3 cell region through their axons. These fibers are called mossy fibers. The axons arising from the CA3 pyramidal cells which synapse onto CA1 pyramidal cell dendrites are called as Schaeffer-collaterals. It is a glutamatergic excitatory transmission. This connection in the hippocampus has provided the most knowledge about the processes of plasticity and basal synaptic transmission.

Originally the hippocampal formation was described as a tri-synaptic pathway: the entorhinal cortex fibers contact the DG granular cells via the perforant path, which themselves give rise to the mossy fibers projecting onto CA3 pyramidal neurons. Through Schaffer collateral axons, CA3 pyramidal neurons in turn form synapses with CA1 pyramidal neurons. Nowadays, the connectivity appears to be more complex since the entorhinal inputs, in fact, project to all hippocampal region (Figure 1), and the Schaffer collaterals do not form a uniform path, but synapse onto CA1 neurons in a highly branched pattern.

The hippocampal formation belongs to the medial temporal lobe system, 
together with the adjacent entorhinal, perirhinal and parahippocampal cortices.

In summary, the strictly laminar organization of the hippocampus makes it a particularly suitable region for electrophysiological recording techniques, both in vitro and in vivo.

Moreover, another advantage of the hippocampal formation is its ability to be transversally cut and maintained in culture for several days, in a so called organotypic slice culture ( $\underline{G}$ ahwiler, 1981) (Stoppini et al., 1991). It has been shown that these cultured slices mimic the developmental time course of this region in situ (D. Muller, 1993) and maintain a connectivity and an expression profile of synaptic proteins comparable to freshly prepared (acute) slices (De

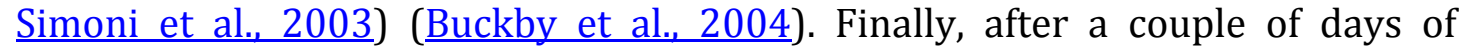
incubation, the slice culture becomes a thin stabilized in vitro system which can be used for electrophysiological recordings. These features make organotypic slices a well-suited system for long-term manipulations. Therefore, during my Ph.D., I decided to take advantage of the hippocampal organotypic slice model to study properties of basal synaptic transmission at CA3-CA1 glutamatergic excitatory synapses.

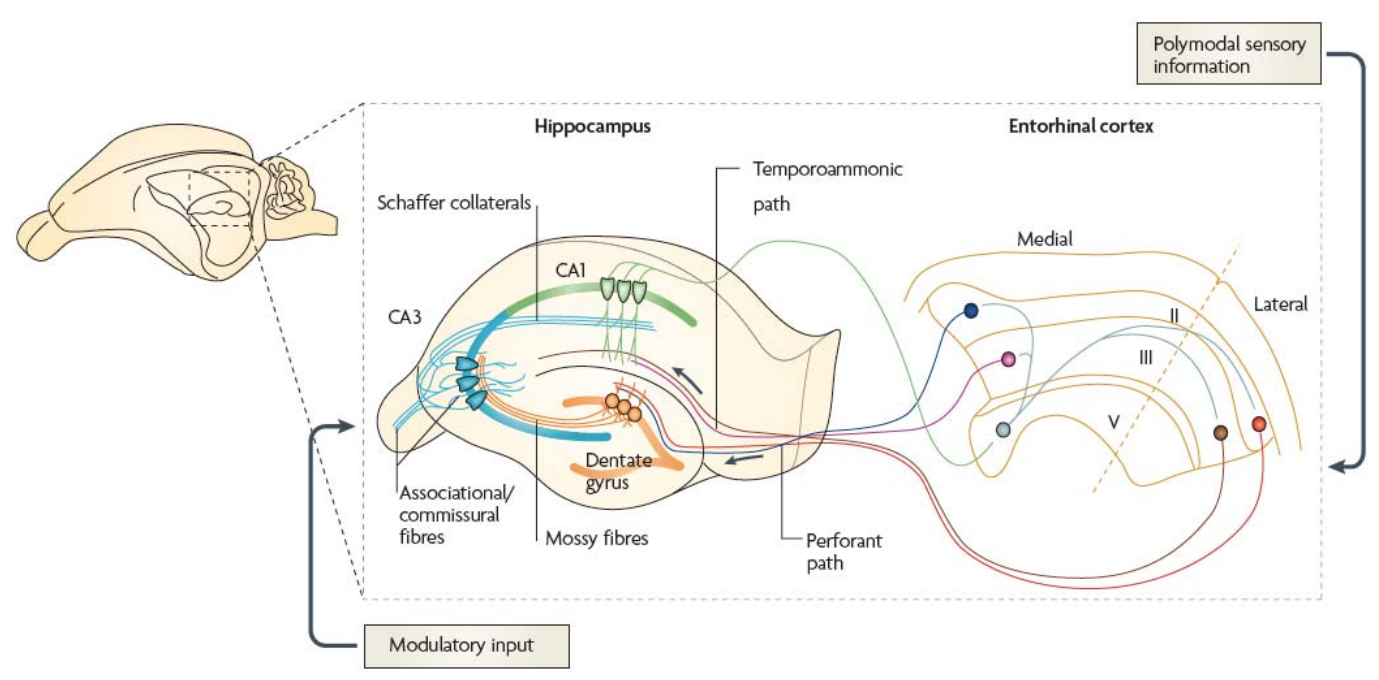

Figure 1. Cartoon depicting the basic anatomy of the hippocampus. 
The schematic shows a transversal section of the hippocampus with its major connections, including inputs from the enthorinal cortices and the basic tri-synaptic intra-hippocampal connectivity. (Modified from (Neves et al., 2008)).

\section{I.B. Glutamatergic Excitatory Synapses}

\section{I.B.1. The Post Synaptic Density}

CA1 pyramidal neurons possess a large triangular cell body shape and dendrites with membrane protuberances called spines. These mushroom shaped specializations face the presynaptic input coming from axon fibers and hold all the necessary machinery to receive and process the information.

In the 1950's, electron microscopy shed light on a fundamental characteristic of the excitatory postsynaptic terminal: it appears as a dense electron structure, which gave rise to the term Post Synaptic Density (PSD) (Figure 2) The PSD is located beneath the postsynaptic membrane and is made of a 30-40 nm thick protein network that comprises a large and dynamic supramolecular assembly ( $\underline{\text { Scannevin and Huganir, 2000) (Yamauchi, 2002) }}$ (Feng and Zhang, 2009). It includes cytoskeleton proteins, adhesion molecules, scaffolding proteins, G proteins and other signaling proteins (Husi et al., 2000) (Kennedy, 2000) (Walikonis et al., 2000). These proteins have the possibility to form a network with different types of receptors, such as glutamate receptors. Depending on whether glutamate receptors are coupled to G protein secondary messenger systems or to the ligand activated cation channels, they are classified as metabotropic or ionotropic glutamate receptors, respectively (Simeone et al., 


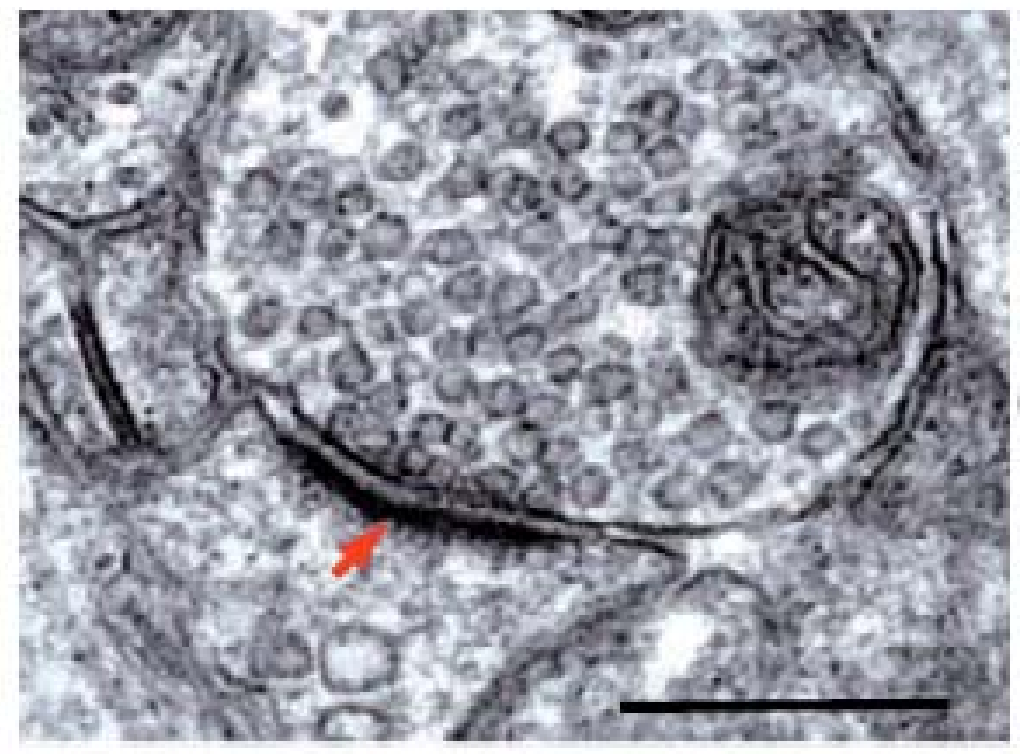

Figure 2. Glutamatergic excitatory synapse visualized by electron microscopy. The presynaptic site is filled with neurotransmitter containing vesicles. The red arrow indicates the post-synaptic density, an electron dense area. (Adapted from (Kennedy, $\underline{2000})$.

\section{I.C. Ionotropic Glutamate Receptors}

Ionotropic glutamate receptors are divided into 3 subgroups, named after the selective agonists with which they were discovered.

o The AMPA ( $\alpha$-amino-3-hydroxy-5-methyl-4-isoxazolepropionic acid) receptor (AMPAR),

0 the NMDA (N-Methyl-D-aspartic acid) receptor (NMDAR),

0 the Kainate receptor (KAR)

(Watkins and Evans, 1981) (Dingledine et al., 1999) (Hollmann and Heinemann, 1994).

Along with a fourth, less characterized, class, the $\delta$ receptors (GluD1-2) 
(Collingridge et al., 2009).

To a large extent, ionotropic glutamate receptors (iGluRs) determine the shape of synaptic currents at glutamatergic synapses. At synapses between CA3 and CA1 hippocampal pyramidal neurons, presynaptic release of glutamate activates primarily two subtypes of ionotropic receptors: AMPARs and NMDARs. AMPARs mediate most of the fast excitatory transmission as NMDAR are blocked by magnesium ions at resting membrane potential.

\section{I.C.1. NMDAR}

NMDARs are heterotetrameric assemblies (Rosenmund et al., 1998) of NR1, NR2(A-D) and NR3(A-B) subunits. NR1 and NR2A mRNA are distributed ubiquitously, with the highest densities occurring in hippocampal regions. In this particular brain region, a functional NMDAR is composed of NR1 with either NR2A or NR2B subunits. Activation of NMDARs requires simultaneous binding of glutamate and a co-agonist (Kleckner and Dingledine, 1988), glycine or D-serine, released by adjacent glial cells (Mothet et al., 2000) (Panatier and Oliet, 2006). The channel is permeable to sodium, potassium and calcium ions. (Burnashev et al., 1996) (Magleby, 2004).

Since the channel pores are blocked by extracellular magnesium in a voltage dependent manner (Novak $L, 1984$ ), channel activation requires a simultaneous release of glutamate by the presynaptic terminal and a depolarization of the postsynaptic membrane which result in the release of the magnesium block. As such, the NMDAR serves as a coincidence detector for coactivation of the pre and the postsynaptic neurons (Figure 3) This detector property together with a high permeability to calcium renders the NMDAR a key player in the process of synaptic plasticity at CA3-CA1 synapses (Harris et al., 1984). One of the most-characterized plasticity processes is long term 
potentiation and its counterpart, long term depression, which take place at CA3CA1 synapses and are NMDAR-dependent. LTP and LTD are expressed by the incorporation or the retrieval of AMPARs at the synapse, respectively ( al., 2000) (Shi et al., 2001) (Lu et al., 2001) (Carroll et al., 2001) (Malinow and Malenka, 2002) hence regulate synaptic strength.

A

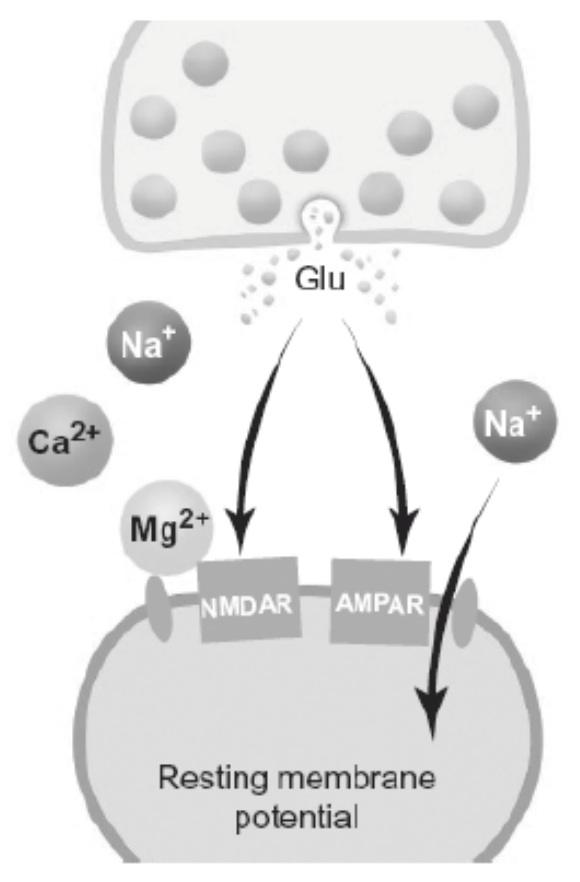

B

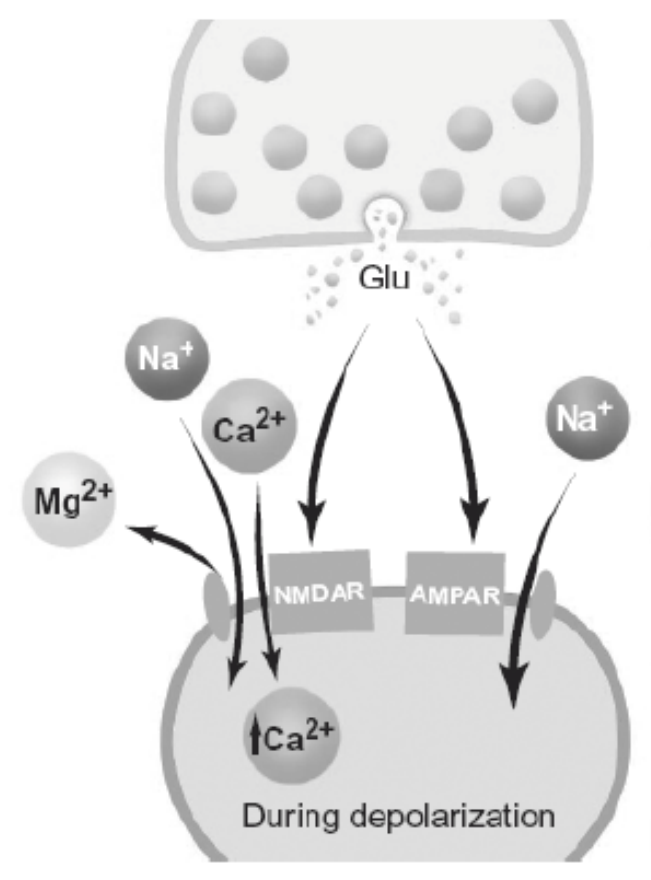

Figure 3. Illustration depicting a glutamatergic synapse.

A) At the resting membrane potential, NMDARs are blocked by extracellular magnesium ions. The release of glutamate by the pre-synaptic terminal activates AMPARs which open and enable sodium to enter the cell. B) A simultaneous postsynaptic membrane depolarization and glutamate release leads to magnesium removal from the NMDARs. NMDAR activation allows sodium and calcium influx into the neuron with an outflux of potassium. (From (Malenka and Nicoll, 1999).

\section{I.C.2. AMPAR}




\section{I.C.2.a. Structure and Composition of AMPARs}

AMPARs are heterotetramers (Mano and Teichberg, 1998) (Rosenmund et al., 1998) composed of four subunits: GluA1, GluA2, GluA3, GluA4 (Dingledine et al., 1999) (Hollmann and Heinemann, 1994), with a maximum of two different subunit types assembled to form a functional receptor (Ayalon and Stern-Bach, 2001) (Mansour et al., 2001). Each subunit contains around 900 amino acids and has a molecular weight of $105 \mathrm{kDa}$ (Rogers et al., 1991). Each subunit possesses a large N-terminal extracellular domain, three transmembrane domains (TM1, 3, 4), one intramembrane domain (TM2), and an intracellular C-terminus. The ligand binds to a pocket created by the association of the extracellular loop between TM3 and TM4 and the N-terminal domain. The C-terminal tail is the binding site of PDZ motif-containing proteins (Figure 4).

\section{Extracellular}

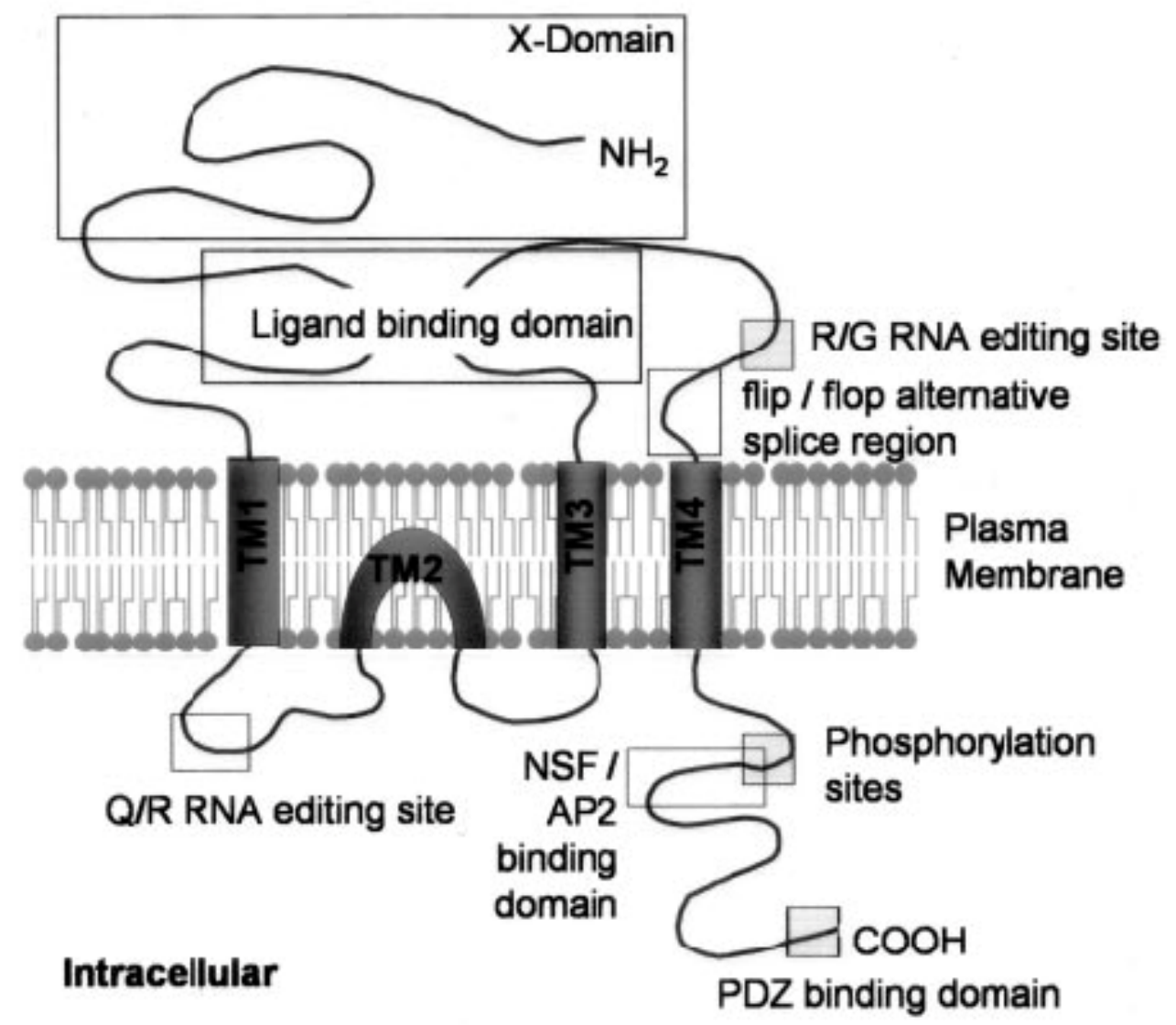


Figure 4. Schematic illustration showing the topology of an AMPA receptor subunit.

The N-terminal domain and the extra-cellular loop form the ligand-binding site. The four transmembrane domains and the intracellular domain are depicted. The alternative splicing sites $(\mathrm{Q} / \mathrm{R}, \mathrm{RG})$, the intracellular interacting proteins or the phosphorylation sites are highlighted by squares. (From (Palmer et al., 2005).

The subunit composition of the receptor varies depending on the brain region. In adult hippocampal pyramidal cells, AMPARs are mainly made of GluA1/GluA2 subunits or GluA2/GluA3 subunits (Wenthold et al., 1996). GluA2 is a necessary subunit for the assembly and the export of the heterotetrameric

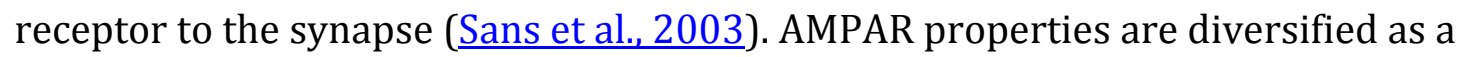
result of post-transcriptional and post-translational modifications.

Each subunit can be alternatively spliced at the beginning of its fourth TM segment, giving rise to the flip and flop isoforms (Sommer et al., 1990), which results in differences in the pharmacological properties of the receptor and the kinetics of the channel. The ratio of each isoform is age-, brain region-, and cell type-dependent. In addition, GluA2 and GluA4 can be alternatively spliced at their intracellular C-terminal tail, giving rise to the short and long isoforms (Gallo et al., 1992) (Köhler et al., 1994). This modification determines the set of interacting proteins, as only short isoforms are able to bind the PDZ motif-

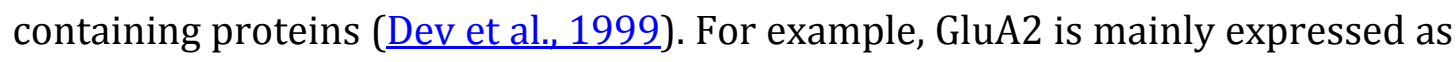
a short isoform ( $90 \%$ ), whereas GluA4 is mainly found as a long isoform.

Moreover, to further diversify the properties of the receptor, RNA editing takes place. One such change is the replacement of a glycine (G) with an arginine (R) upstream of the flip/flop domain. This change affects the desensitization and resensitization properties of the receptor, and occurs mainly in the adult brain on GluA2, GluA3, and GluA4 subunits (Lomeli et al., 1994). A second RNA editing 
site converts a glutamine $(\mathrm{Q})$ into an arginine $(\mathrm{Q} / \mathrm{R})$, but this change is limited to

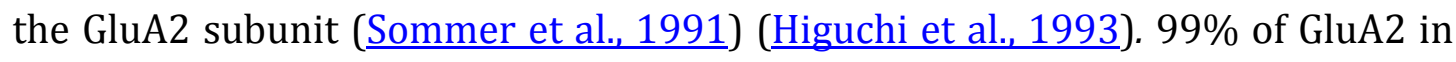
the adult brain exhibits this modification. This change in GluA2 mRNA coding affects the ion channel pore region and renders GluA2-containing AMPA receptors permeable only to monovalent cations (observing a linear current/voltage curve) (Verdoorn et al., 1991), whereas GluA2-lacking receptors are capable of conducting calcium ions as well.

Finally, in addition to these putative amino acid modifications, AMPARs contain multiple phosphorylation sites, with each subunit having its own phosphorylation pattern. These patterns determine the biophysical properties of the receptors as well as its interacting partners and its cellular trafficking (Wang et al., 2005). For example, the GluA1 cytosolic tail hosts phosphorylation sites for CaMKII (Calcium/Calmodulin Kinase II) at serine 831 and another one for PKA (Protein Kinase A) at serine 845 (․ㅡㄹ et al., 1996) (Barria et al., 1997) (Mammen et al., 1997). During basal synaptic transmission, the PKA site is phosphorylated, whereas the CaMKII site is not. Moreover, it was shown that serine 845 phosphorylation by PKA increases the channel opening probability (Banke et al., 2000), and phosphorylation of serine 831 by CaMKII enhances the channel conductance (Derkach et al., 1999). Therefore, during synaptic plasticity, the GluA1 biophysical properties are modified by a combination of specific phosphorylation patterns according to the synapse history.

\section{I.C.2.b. AMPAR biosynthesis and trafficking}

Before the modification of AMPAR function at the synapse, how do the receptors reach this specialized compartment?

First of all, it is known that AMPAR are synthesized, maturated (folding, Nglycosylation, disulfide bond) and assembled in the endoplasmic reticulum (ER) in the soma. Only properly folded and assembled receptors are then exported to the Golgi apparatus (Greger et al., 2002). The non-conventional receptors are 
kept in the reticulum and degraded through the proteasomal pathway (Vandenberghe and Bredt, 2004). Properly folded receptors are transported to dendrites through motor proteins such as myosin, dynein or kinesins (Hirokawa and Takemura, 2005) (Bridgman, 2004), which bind to microtubules or actin filaments. This trafficking process involves adaptor proteins as well as scaffolding proteins to create a link between the receptor and the motor proteins (Setou et al., 2002) (Wu et al., 2002) (Braithwaite et al., 2000) (Bredt and Nicoll, 2003). In this way, receptors can travel long distances in neurites when associated with microtubules, or shorter distances (like in the spine) when bound to actin. Moreover, trans-golgi apparatuses are also present in dendrites (Pierce et al., 2001) (Horton and Ehlers, 2003), where local synthesis can take place in response to synaptic activity (Ju et al., 2004) (Sutton et al., 2006).

A very specific characteristic of AMPARs is their ability to be constitutively recycled at the membrane (Carroll et al., 1999) within a 10-30 minute time frame (Ehlers, 2000) (Passafaro et al., 2001). Different studies show that the endo and exocytosis sites are localized in the spine itself, next to the post synaptic density (Blanpied et al., 2002) (Petralia et al., 2003) or in the dendritic shaft (Passafaro et al., 2001) (Park et al., 2006) (Yudowski et al., 2007) (Lin et al., 2009). How do AMPARs travel between these two sites (synaptic and extrasynaptic)? This leads to the next feature of AMPARs: they are capable of freely diffusing along the cell membrane (Figure 5) (Borgdorff and Choquet,

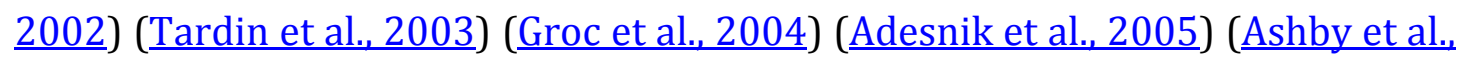
2006) (Isaac et al., 2007) (Bats et al., 2007). In addition, it has been shown that GluA2 is constitutively inserted at the synapse, while GluA1 is inserted in an

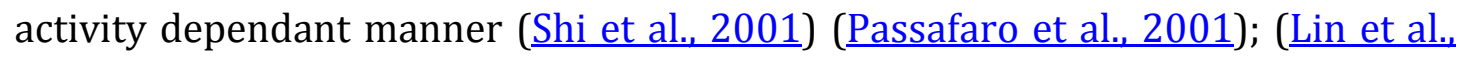
2009). Thus, the GluA1 subunit dictates the AMPAR insertion mode. The different trafficking rules for GluA1 and GluA2 correspond to the differences in the structure of their cytosplasmic tails. Altogther, this suggests a sensitive finetuning process, and raises the question of what is regulating AMPAR trafficking to the synapse? 


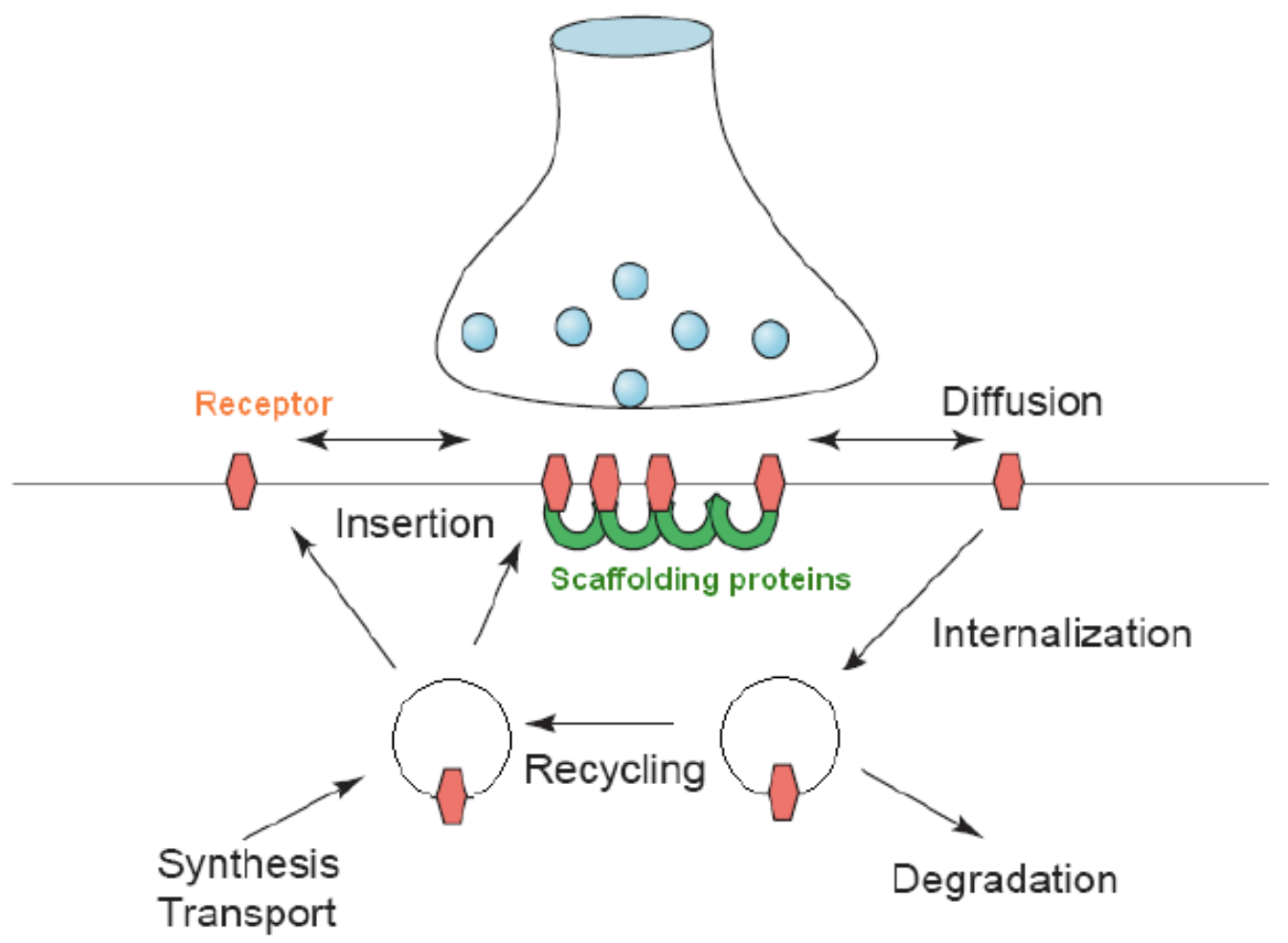

Figure 5. AMPARs are exchanged between synaptic, extra-synaptic and intracellular compartments.

AMPARs can diffuse along the neuronal membrane, but can be trapped and stabilized at synapses through scaffolding protein interactions at the postsynaptic site. (From (Triller and Choquet, 2005).

\section{I.C.2.c. AMPAR modulating proteins}

During the last decade, a concept emerged, suggesting that AMPARs are the center of a multiprotein complex (Jackson and Nicoll, 2011). These interactions regulate numerous aspects of AMPAR properties. Thus, AMPAR biophysical properties are based not only on the molecular identity of the receptor (subunit composition, splice variation, RNA editing, and posttranslational modifications) but also on the proteins interacting with AMPARs, to build an AMPAR complex that is important for moment-to-moment neuronal 
communication.

\section{I.C.2.c.i. TARPS}

The first group of proteins identified as AMPAR auxiliary subunits, as they are able to regulate AMPAR biophysical properties, are called TARPs, Transmembrane AMPAR Regulatory Proteins (Kato et al., 2010b).

The prototypical and first discovered TARP was stargazin. It was revealed by the analysis of a spontaneous mutation that causes absence epilepsy, head tossing, and ataxia (Noebels et al., 1990). The mutant mouse keeps looking at the sky, hence the protein responsible for the phenotype was named as stargazin and the mouse as "stargazer". Due to its $25 \%$ homology with the skeletal muscle calcium channel subunit, $\gamma$-1 (Jay et al., 1990), the peptide was first named as $\gamma$ 2. In fact, its gene symbol, Cacng2, is also derived from Cacng1 skeletal muscle gene nomination, which encodes for $\gamma-1$. However, nowadays, stargazin is its more common name.

The stargazin locus encodes a $38 \mathrm{kDa}$ protein which is a four-pass transmembrane protein with both $\mathrm{N}$ and C-termini in the cytosolic region. Absence of stargazin is associated with selective and complete loss of AMPAR function in cerebellar granule cells (Chen et al., 2000) (Hashimoto et al., 1999). At the cellular level, different sets of experiments showed that stargazin promotes synaptic targeting of the AMPAR (Chen et al., 2000) (Tomita et al., 2003) (Vandenberghe et al., 2005) in a two-step model. First, by bringing AMPARs to the neuronal plasma membrane, stargazin ensures their proper maturation through the secretory pathway. Second, through its C-terminal interaction with PDZ-containing proteins, stargazin retains AMPARs at the PSD. In addition, stargazin also modulates receptor pharmacology and controls channel gating and the permeability of the receptor. Specifically, stargazin enhances AMPAR glutamate affinity, and therefore delays deactivation and 
desensitization of the receptor. Moreover, stargazin attenuates intracellular polyamine block of calcium-permeable AMPARs (which is dictated by the $Q$ residue in GluA2-lacking subunits), thus increasing the current at depolarized

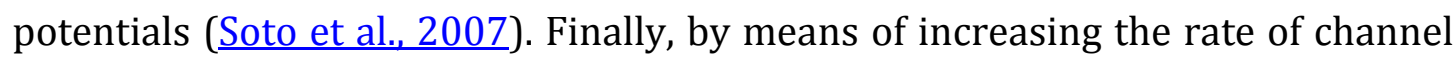
opening, and thus single-channel conductance (Tomita et al., 2005a) (Tomita et al., 2005b) (Soto et al., 2007), stargazin participates in modulating AMPAR transmission with its ability to dissociate from AMPARs after they bind glutamate (Tomita et al., 2004). This interaction provides a mechanism to prevent excitotoxicity.

Phylogenic analysis revealed that TARPs could be divided into subgroups based on their sequence homology. Therefore, stargazin belongs to the type I group of TARP isoforms along with three other members: $\gamma-3, \gamma-4$, and $\gamma-8$. Type II consists of $\gamma-5$ and $\gamma-7$ (Tomita et al., 2003). All TARPs are differentially expressed throughout development and display distinct regional specificity as well as expression patterns according to neuronal and glial cell populations ( $\underline{\text { Cho }}$ et al., 2007) (Fukaya et al., 2006) (Kato et al., 2007) (Milstein et al., 2007); (Moss et al., 2003) (Soto et al., 2009) (Tomita et al., 2003). For example, $\gamma-8$ is predominant in the telencephalon, with the highest level in hippocampus. But, $\gamma$ $2 /$ stg, $\gamma-3$, and $\gamma-4$ are still co-expressed (though at a lower level).

TARPs bring a specific regulation onto the AMPAR properties which is a highly versatile and complex process and thus is currently under intensive investigation. To summarize, type I TARPs associate with all four GluA subunits and regulate AMPAR trafficking, gating, and pharmacology in a TARP subtypespecific manner (Nicoll et al., 2006) ( Osten and Stern-Bach, 2006) (Ziff, 2007). Moreover, different expression levels of the different TARP isoforms change the TARP/AMPAR stoichiometry (Kim et al., 2010) (Shi et al., 2009), which diversifies AMPAR functional properties further.

\section{I.C.2.c.ii. CNIH}


As social networking seems to be a key behavior of our generation, AMPARs too appear to interact with a growing number of candidate proteins. In

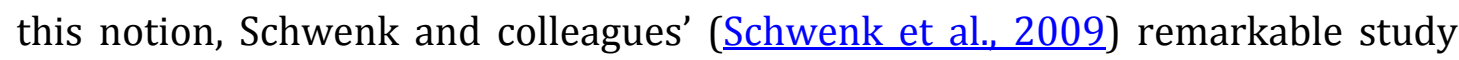
revealed that, in addition to interacting with TARPs, AMPARs also associate with CNIH-2 and CNIH-3 which are vertebrate homologs of Drosophila cornichon (French for "pickled gherkin"). This topic is under active research and is thus subject to competing theories, such as whether AMPAR interacting partners are mutually exclusive (Schwenk theory) or form a tripartite complex composed of AMPAR/TARPs/CNIH (Kato et al., 2010a). However, there is consensus that $\mathrm{CNIH}$ acts as a bonafide auxiliary subunit and regulates AMPAR (GluA1) trafficking, channel properties and pharmacology. Cornichons increase surface expression of AMPARs and slow down deactivation and desensitization kinetics.

\section{I.C.2.c.iii. CKAMP44}

More recently, another AMPAR interacting partner has been identified by Von Engelhardt (von Engelhardt et al., 2010): the protein CKAMP44 (cysteineknot AMPA receptor modulating protein of $44 \mathrm{kDa}$ ). It has been shown that CKAMP44 has an effect opposite to TARPs in terms of surface trafficking and desensitization (CKAMP44 accelerates desensitization). They showed that slowing down the recovery from desensitization attenuates facilitation of the postsynaptic response at lateral and medial perforant path granule cell synapses. Interestingly, CKAMP44 appears to be poorly expressed in CA1neurons but has a robust expression level in dentate gyrus granular cells. Due to this observation, it has been suggested that CKAMP44 modulates short-term plasticity at different synapses.

In summary, various mechanisms regulating AMPAR kinetics have already been unraveled, and certainly many more are going to be revealed in the future. All 
with the same aim, understanding the synapse-specific communication and the fidelity of synaptic transmission. The next step consisted to understand what is holding AMPARs at the synapse.

\section{I.D. DLG-MAGUK}

AMPARs are maintained at the synapse via their interaction with scaffolding proteins anchored at the PSD (Wyszynski et al., 1999) (Braithwaite et al., 2000). These scaffolding proteins possess PDZ (PSD-95/Discs large homolog/Zona occludens-1) domains, as do $\sim 400$ other proteins expressed in the rodent species ( $\mathrm{Kim}$ and Sheng, 2004). The PDZ domain is a $\sim 90$-residuelong module that typically binds short peptide motifs ( $\sim 4-6$ residues) at the extreme C-terminal end of its interacting partners but also have the possibility to heterodimerize (Sheng and Sala, 2001). PDZ domains tend to fall into three categories based on their interaction affinity with different ligands (Marfatia et al., 1997, Marfatia et al., 2000) (ㅂsueh et al., 2000) (Jo et al., 1999). PDZ domains are usually found in tandem, which creates the possibility for a high protein concentration at a specific spot, helping to build macromolecular complexes. At the PSD, numerous PDZ domain-containing proteins are involved in the structural organization of the synapse (Kim and Sheng, 2004). The most abundant PDZ domain proteins at the post synaptic density are the MAGUKs (Membrane Associated GUanylate Kinases), more specifically the subtype DLGMAGUKs (Kim and Sheng, 2004) (Funke et al., 2005) (Elias and Nicoll, 2007).

The DLG-MAGUK family is the rodent and human homolog of the Drosophila discs large tumor suppressor protein (DLG-MAGUKs).

The members of this family include:

o PSD-95 (Cho et al., 1992) (Kistner et al., 1993),

o PSD-93 (마renman et al., 1996) ( 
o SAP97 (Lue et al., 1994) (Müller et al., 1995) and

o SAP102 (üller et al., 1996).

\section{I.D.1. Structural organization of the DLG-MAGUKs}

The hallmark of all DLG-MAGUKs is the presence of the same domain topology, composed of five modular protein interaction domains, starting from the N-terminus with three PDZ domains, followed by an SH3 (src-homology 3) domain next to a catalytically inactive GK (guanylate kinase) domain (Figure 6) (Garner and Kindler, 1996, Montgomery et al., 2004) (Montgomery et al., 2004) (Olsen and Bredt, 2003).

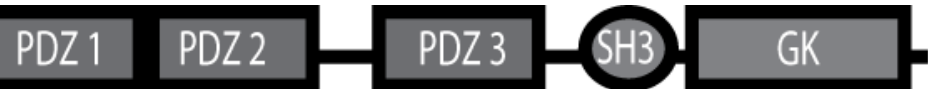

Figure 6. Schematic diagram of PSD-95 domains showing the modular protein interaction motifs including three PDZ domains in tandem, an SH3 domain and a GK domain.

The GK domain is catalytically inactive as a result of mutations to key residues involved in the nucleotide binding and transition state stabilization (Kuhlendahl et al., 1998). In its typical form, SH3 domain is characterized by its ability to bind to proline rich stretches. However, in the DLG-MAGUKs SH3 domain, the Hinge/Hook region (between SH3 and GK domain) occupies the binding pocket of the SH3 domain. The SH3 and GK domains interact in an intra-molecular manner (Figure 7A), but the functional significance of this interaction is unclear (McGee and Bredt, 1999, Tavares et al., 2001), (Shin et al., 2000), (Newman and Prehoda, 2009). Moreover, it has been proposed that DLG-MAGUKs can interact among each other in an inter-molecular fashion whereby the SH3 domain of one DLG-MAGUK could interact with the GK domain of another DLG-MAGUK and vice 
A.

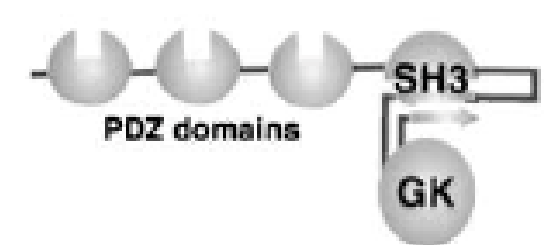

B.

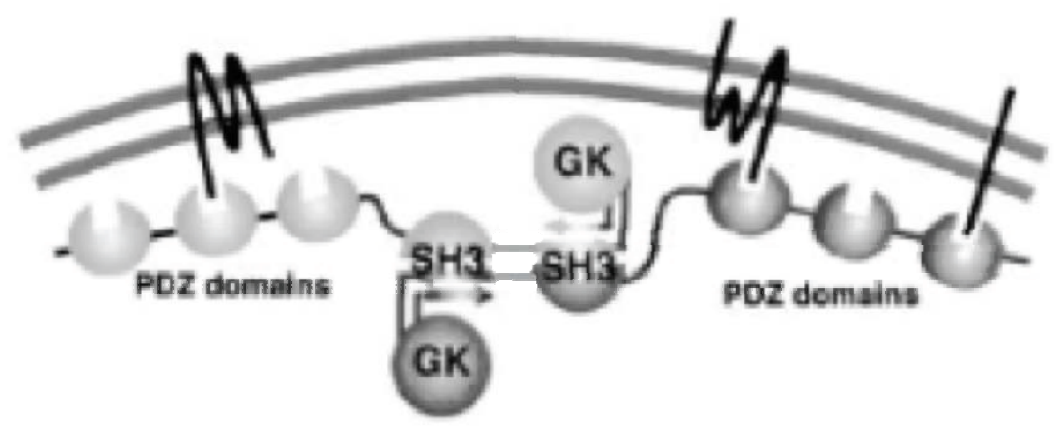

Figure 7. Illustration of an SH3-GK intra- and inter-molecular interactions.

A) Intra-molecular interaction between the SH3 domain and GK domain of the same DLG-MAGUK. B) Inter-molecular interaction between differents DLG-MAGUKs using the SH3-GK module. (From (McGee et al., 2001))

Despite the high homology among the four members, the DLG-MAGUKs display differences in their $\mathrm{N}$-terminal part due to alternative splicing. On the protein level, these sequence variations result in either a palmitoylation sequence (Topinka and Bredt, 1998) or a L27 domain found upstream the first PDZ domain (Lee et al., 2002) (Nakagawa et al., 2004), named alpha and beta isoforms, respectively.

- The L27 domain got its name by virtue of the same motif found in Lin-2 and Lin-7 C.elegans proteins, which allows the protein to dimerize (Chetkovich et al., 2002) (Lee et al., 2002). This splice variant has been reported for PSD-93, PSD-95 and SAP97. In the case of the last two, the 
beta isoform influences AMPA receptor-mediated synaptic strength in an activity-dependant manner ( $\underline{\text { Schlüter et al., 2006) }}$.

- On the other hand, the alpha-isoform contains characteristic cysteine residues, which can be modified by the addition of the fatty acid, palmitate, by palmitoyl transferases. In the case of PSD-95, palmitoylation of the cysteines in position 3 and 5 have been shown to be necessary for synaptic targeting and synaptic clustering (Craven et al., 1999) (ElHusseini et al., 2000a) (Topinka and Bredt, 1998). Moreover, palmitoylation allows PSD-95 to multimerize with itself ( $\underline{\text { Hsueh and }}$ $\underline{\text { Sheng, 1999) }}$ or with other palmitoylated proteins such as PSD-93 (ㅍueh et al., 1997). Finally, in contrast to the beta-isoform, this splice variant has been shown to be activity-independent ( $\underline{\text { Schlüter et al., 2006) }}$.

SAP102 has a unique N-terminal region that forms a zinc finger (El-Husseini et al., 2000c), and PSD-93, in addition to its alpha and beta isoforms, possesses four others splice variants (Parker et al., 2004) (Brenman et al., 1996).

The alpha-isoform is the major isoform of PSD-95 (Chetkovich et al., 2002), while SAP97 is mainly expressed in its beta-isoform (Schlüter et al., 2006)

\section{I.D.2. Distributions of the DLG-MAGUKs}

The four members of the DLG-MAGUK family are subjected to distinct spatiotemporal expression patterns during brain development (Sans et al., 2000). In the rodent brain, SAP102 is the first DLG-MAGUK expressed at the late embryonic stage and reaches its expression plateau around post-natal day 10 (P10), whereas the three other members are gradually increased during postnatal development ( $\underline{\text { Sans et al., 2000 }}$ Sans et al., 2001). The DLG-MAGUK constituents are present at different sub-cellular localizations. While PSD-95 and PSD-93 are enriched in the PSD (hen et al., 2005) (Cheng et al., 2006) ( et al., 2003) (Valtschanoff and Weinberg, 2001), SAP97 and SAP102 were at first suggested not to be (El-Husseini et al., 2000c) (Waites et al., 2009). SAP97 was at first suggested to exist in a more diffused somato-dendritic localization (Müller 
et al., 1995). However, the synaptic enrichment of SAP97 is debated by some groups observing it (Chetkovich et al., 2002) (Rumbaugh et al., 2003, Nakagawa et al., 2004). The discrepancies observed between the studies might result from different expression levels, as the studies by Chetkovich, Rumbaugh and Nakagawa used transfection, which results in much higher expression than the lentiviral-mediated transduction used in the Waites' study. SAP102 is suggested to have an intermediate distribution--present along the spine (PSD and cytoplasm) and dendrite. Using immunogold double staining (PSD-95 and SAP102), Zheng and colleagues (Zheng et al., 2010) showed that in hippocampal dissociated neuron cultures (E18) transfected on the 18th day in vitro (DIV) with PSD-95 and SAP102 and fixed at DIV21-22, show a PSD-95 enrichment at the upper part of the PSD (close to the membrane), while SAP102 is present mainly in the lower part of the PSD (further away from the membrane) with a colocalization of both proteins in the middle section of the PSD. Moreover, the authors showed that the majority of SAP102 is highly mobile in spines, whereas the majority of PSD-95 is immobile.

\section{I.D.3. Functions of PSD-95}

\section{I.D.3.a. Role of PSD-95 in synaptic maturation}

During development, PSD-95 is suggested to influence synaptic maturation. PSD-95 overexpression in hippocampal dissociated cultures accelerates development, promotes spine enlargement and increases the number of spines (El-Husseini et al., 2000b). Moreover, in this study, a selective enhancement of GluA1, but not NR1, clustering was observed. This correlates with previous studies showing that the number of NMDARs remains relatively constant, whereas the number of synaptic GluAs increases during development. However, very interestingly, PSD-95 has been shown to interact directly with NMDARs (Kornau et al., 1995) (Niethammer et al., 1996) (through its PDZ domains with the C-terminal part of NMDAR), while it does not interact directly 
with AMPARs. This discrepancy in the results was explained by the discovery of a common protein binding both AMPAR and PSD-95, stargazin (Chen et al., 2000). AMPAR C-terminals bind stargazin. In turn, the stargazin C-terminal PDZ-binding site associates with the PDZ 1 and 2 domains of PSD-95 (Dakoji et al., 2003).

\section{I.D.3.b. Role of PSD-95 in synaptic plasticity}

Additionally, PSD-95 interacts with a wide range of scaffold proteins, actin cytoskeleton components and cytoplasmic signaling molecules (Sheng and Kim, 2002) (Husi et al., 2000) (Husi and Grant, 2001) (Kim and Sheng, 2004) (Montgomery et al., 2004) ( (Scannevin and Huganir, 2000). Consequently, PSD-95 has the capacity to bring signal-transducing enzymes in proximity with surface receptors, facilitating signal transduction within the PSD to the downstream pathway. For these reasons, PSD-95 earned the title of signaling scaffold protein. As befits an important signaling scaffold protein of the PSD, PSD-95 has a large influence on synaptic plasticity (Migaud et al., 1998) (Yao et al., 2004) (Béïque et al., 2006). PSD-95 has been suggested to regulate NMDAR-dependent synaptic plasticity by acting as a "slot" protein to anchor AMPARs at the synapse ( $\underline{\text { Schnell }}$ et al., 2002). Indeed, levels of PSD-95 have been correlated with the strength of synaptic transmission. In addition, immunocytochemistry experiments suggested that the enhancement in AMPAR transmission is related to an increase in the numbers of AMPARs present at the PSD, rather than a change in biophysical properties of the AMPARs (El-Husseini et al., 2000b).

\section{I.D.3.c. Role of PSD-95 in synaptic AMPAR function}

\section{I.D.3.c.i. Overexpression of PSD-95}

The fact that overexpression of PSD-95 drives a selective and robust increase in AMPAR transmission (but not in NMDAR transmission) ( $\underline{\text { Schnell et al., }}$ 2002) (Ehrlich, 2004) ( Schlüter et al., 2006) suggests another potential role of 
PSD-95 in addition to the signaling scaffold associated with NMDARs. It was proposed that PSD-95 has the capacity to regulate the number / function of AMPARs at the synapse, acting as a "slot" protein to anchor AMPARs at the synapse, and thus regulating basal synaptic transmission ( $\underline{\text { schnell et al., 2002). }}$.

\section{I.D.3.c.ii. PSD-95 knockouts}

One way to establish the necessity of a protein in a biological function is to delete the protein of interest and assay a specific function in its absence. For such purpose, germline truncations/deletions of MAGUKs were produced, giving rise to single or double knockout (KO) mice.

The first gene targeted truncation of PSD-95 was produced by Migaud et al (Migaud et al., 1998), and, very surprisingly, showed normal AMPAR and NMDAR-mediated transmission. It should be noted that, in this study, a mouse carrying a stop codon after PDZ1 and PDZ2 domains was generated. Hence, the targeted mutation in the PSD-95 gene led to the expression of a truncated protein containing PDZ1 and PDZ2 domains in frame with an IRES (internal ribosome entry site) b-galactosidase gene reporter (mouse line named PSD-95 PDZ12). Nevertheless, an important feature of this mouse line is the absence of PSD-95 PDZ12 protein in the PSD fraction, in synaptosomes and in synaptic plasma membranes of homozygote mice. This is in contrast to the full length PSD-95, which can be detected in any of these compartments in wild type mice.

In an attempt to rule out the possibility that the truncated PSD-95 (PSD95 PDZ12) could still participate in basal synaptic transmission in this PSD-95 PDZ12 KO animal, Yao et al (Yao et al., 2004) generated another PSD-95 KO mouse line. This mouse line was named as PSD-95-GK as it targets a deletion of the GK domain in the PSD-95 gene. Mice carrying the this truncated form of PSD95 gene do not produce any detectable PSD-95 proteins (Yao et al., 2004) (Elias et al., 2006) yet displaying an unaltered AMPAR and NMDAR transmission (Elias et al., 2006) (Elias et al., 2008).

Interestingly, in another line of PSD-95 KO mice carrying a complete PSD- 
95 gene deletion, a defect in AMPAR transmission was observed when recordings were performed between P14 and P24 (but not in younger animals) (Béïque et al., 2006).

This discrepancy concerning an altered basal synaptic transmission in the different KO lines could be related to the mouse line itself or to reflecting a different maturation state of the synapses under investigations. Moreover, the normalcy of synaptic transmission observed in certain KO lines could be related to a functional compensation mediated by the other DLG-MAGUKs due to their molecular redundancy. In an attempt to limit possible compensatory mechanisms, the shRNA technology was used. shRNA methods have an advantage in the fact that it functions on an acute timescale, does not completely abolish the protein in the neuron and its expression can be temporally limited to an already mature neuron and thus a mature synapse.

\section{I.D.3.c.iii. shRNA against PSD-95}

Knockdown of PSD-95 by means of RNA interference technology showed a 50\% decrease in AMPAR EPSC with no effect on NMDAR EPSC in most studies (Elias et al., 2006) (ㄷhlüter et al., 2006, Elias et al., 2008). However, it should be noted that the Ehrlich et al paper (Ehrlich et al., 2007) also observed a small but yet significant decrease in NMDA transmission. As already mentioned above, the opposite manipulation (pure over-expression of PSD-95) mainly leads to a $\sim 2$ to 3 fold increase in the amplitude of AMPAR mediated EPSCs with no effect on the NMDA component (El-Husseini et al., 2000b, Schnell et al., 2002) (Ehrlich, 2004)

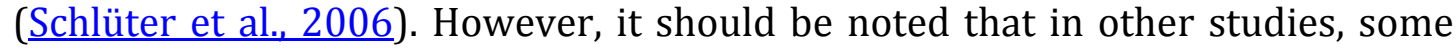
upgrade of NMDAR transmission was also observed (Kim et al., 2007) (담ai et al., 2007).

These observations point to a critical role for PSD-95 in terms of regulating basal 
synaptic transmission.

\section{I.D.3.d. Roles of PSD-95 domains in basal synaptic transmission}

After establishing the importance of PSD-95 in the regulation of basal synaptic transmission, the next interesting step focuses on determining the role of different domains in this process.

With this aim, Craven et al. (Craven et al., 1999) underscored the requirement of the N-terminal part of the protein for synaptic targeting and enrichment. They revealed that the N-terminus of PSD-95 allows a head-to-head interaction to prompt multimerization (Hsueh and Sheng, 1999). Moreover, the N-terminal palmitoylation appears to be a prerequisite for its synaptic targeting (Craven et

al., 1999). In addition, electrophysiology studies based on overexpression of specific truncated forms of the protein highlighted the necessity of the PDZ1 and

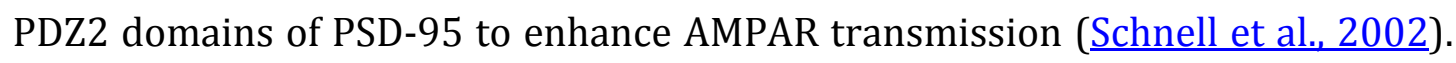
These data were corroborated by confocal microscopy imaging, showing a typical punctate distribution, corresponding to the PSD enrichment of the protein (Craven et al., 1999).

It should be noted that the study by Migaud et al (Migaud et al., 1998) and by Schnell et al ( $\underline{\text { Schnell et al., 2002) }}$ are in clear contrast. Althought, both used the truncated PSD-95 PDZ12 protein, the former sees no change in AMPARtransmission, accompanied by an absence of the mutant protein from the synapse. The latter sees an increase in AMPAR-transmission concomitant with a typical punctuate pattern of a fully functional PSD-95.

The difference in the results is explained by the absence of the endogenous PSD95. Indeed, in the experimental set-ups, Schnell et al. (Schnell et al., 2002) overexpressed PSD-95 PDZ12 in neurons already expressing full-length PSD-95, while Migaud et al. (Migaud et al., 1998) expressed PSD-95 PDZ12 in KO neurons. As stated above, PSD-95 has the capacity to multimerize with itself. Therefore the endogenous PSD-95 can team up with the PSD-95 PDZ12 and mediate the 
function of AMPAR transmission in duo.

Due to the importance of the background environment in the neuron, (i.e., expressing or not expressing endogenous PSD-95) to distinguish the results, a

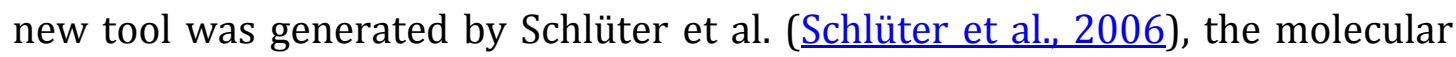
replacement method. It consists of knocking-down the endogenous protein and replacing it with a mutant under investigation (see section IV.A.2 for more details).

Consequently, the minimal requirement of PSD-95 to influence basal synaptic transmission was revisited using the so-called molecular replacement method.

In addition to the N-terminal part and the two first PDZ domains, previously defined as the minimal domains necessary for PSD-95 to influence

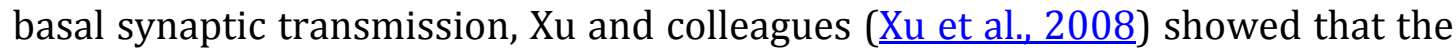
SH3-GK module is an additional requirement. Removing the C-terminal part of PSD-95 (SH3 and GK domains, named PSD-95 $\Delta$ SH3-GK) and overexpressing it in wild-type neurons, produced an enhanced AMPAR transmission. However, such an enhancement was not observed when using the molecular replacement technique. Indeed, knocking-down endogenous PSD-95 via shRNA and replacing it by PSD-95 $\Delta$ SH3-GK led to a $\sim 50 \%$ decrease in AMPAR-currents amplitude. This shows that, as already pointed-out before, the background environment in a neuron is important in terms of the presence of the endogenous proteins. Moreover, this demonstrates that PSD-95 lacking the SH3-GK module is not functional, additional requirement of these domains for proper PSD-95 function. In addition, these electrophysiological findings were supported by confocal microscopy data, showing a more diffused somato-dendritic pattern of the PSD95 $\Delta$ SH3-GK instead of the typical punctate pattern observed with full length PSD-95 ( $\underline{\mathrm{Xu} \text { et } \mathrm{al} ., 2008}$ ). This experimental background generated the motivation for my current Ph.D. project, and provided a starting point. 


\section{I.E. Scope of the thesis}

For my thesis I set out to determine which of the SH3 and/or GK domains are needed to mediate, in partnership with the N-terminal amino acids and the two first PDZ domains, the regulation that PSD-95 exerts on basal synaptic transmission.

To this end, I choose the hippocampal organotypic slice preparation as a model system and used a combination of molecular replacement and electrophysiological techniques. This involved molecular cloning of the mutant forms of PSD-95 lacking different sets of domain(s) in a lentiviral vector, generation of the lentivirus, injection of the lentiviral particles into CA1 pyramidal cell layers and then performing dual whole-cell patch-clamp experiments. The electrophysiological properties between a neighboring control neuron and an infected neuron expressing mutant PSD-95 were compared. By deleting a single domain (or multiple domains) of PSD-95, I could assess if the mutant protein was able to rescue the deficit in basal synaptic transmission mediated by shRNA against the endogenous PSD-95. This allowed me to evaluate the participation of certain domains of PSD-95 for their ability to functionally influence synaptic strength. 


\section{Materials and Methods}

\section{II.A. Materials}

The mentioned companies in the below sections are referred as such:

AGCT DNA Core Facility, Max Planck Institute of Experimental Medicine, Göttingen, Germany.

A.M.P.I. , Jerusalem, Israel.

Axon instrument, Sunnyvale, CA, USA.

Ascent Scientific, Bristol, UK.

Beckman Instruments GmbH, Munich, Germany.

Biochrom, Berlin, Germany.

Bioline, Luckenwalde,Germany.

BioLog, Bremen, Germany.

Chemicon, Temecula, CA, USA.

DAGE-MTI, Michigan City, IN, USA.

Drummond Scientific, Broomall, PA, USA.

Eppendorf AG, Hamburg, Germany.

Fermentas, St. Leon-Rot, Germany.

Finnzymes, via Biozym Scientific GmbH, Oldendorf, Germany.

Greiner Bio-One, Frickenhausen, Germany.

Hamamatsu, Herrsching, Germany.

Harnischmacher, Fröndenberg, Germany.

HyClone/Thermo Fisher Scientific, Waltham, MA, USA.

Intas, Göttingen, Germany.

InstruTECH/HEKA, Lambrecht, Germany.

Invitrogen / Molecular Probes / Gibco, Karlsruhe, Germany.

Invitrogen/Life Technologies, Darmstadt, Germany.

King Precision Glass, Inc., Claremont, CA, USA.

Labotech, Cape Town, South Africa.

Li-COR Biosciences, Bad Homburg, Germany. 
Merck/VWR, Darmstadt, Germany.

Millipore, Schwalbach/Ts., Germany.

Mirus Bio, Madison, WI, USA.

Molecular Devices, Sunnyvale, CA, USA.

New England Biolabs (NEB), Frankfurt, Germany.

Npi, Tamm, Germany.

New Brunswick scientific / Eppendorf AG, Hamburg, Germany.

PAA, Germany.

5-Prime, Hamburg, Germany.

Qiagen, Hilden, Germany.

Roche, Mannheim, Germany.

Roth, Karlsruhe, Germany.

Sartorius, Goettingen, Germany.

Sigma, Munich, Germany.

Sigma-Aldrich, München, Germany.

Sigma-Genosys, Steinheim, Germany.

Stratagene, Santa Clara, CA, USA.

Sutter Instrument, Novato, CA, USA.

Uptima interchim via VWR international, St Augustin, Buisdorf, Germany.

Whatman/GE Healthcare, Maidstone, UK.

WaveMetrics Inc., Lake Oswego, OR, USA.

Wescor ,Logan, Utah, USA

World Precision Instruments, Sarasota, FL, USA.

Worthinghton

Chemical /commercial reagents and kits

Supplier

Adenosine 5í'- triphosphate (ATP)

Sigma

Agarose

Sigma

A.M.B.A

Roth

Ampiciline

Roth

A.P.S (10\%)

Roth

l-Ascorbic acid

Sigma

Aspartate (amino-acids)

Roth

d-APV

Ascent Scientific

Bacteria Escherichia coli (E. coli),

XL1-Blue

Stratagene

TOP10

Invitrogen

B27

BME (basal medium + Earle's)

Invitrogen

Biochrom 
Bicucculine

Bromophenolblue

BSA

$\mathrm{CaCl} 2$

$\mathrm{CaCl} 2 * 2 \mathrm{H} 2 \mathrm{O}$

2-chloroadenosine

Chloroform

L-Cysteine

DMEM/HamsF12

DNaseI

dNTPs

DiThioThreitol

EDTA

Enzymes

Ethidium bromide

FUDR

d-Glucose

d-Glucose*H2O

Glutamate (amino acids)

Glutamax

Glycerol

HEPES

Horse serum

HBSS (Hanks balanced salt solution 10x) + $\mathrm{MgCl} 2,+\mathrm{CaCl} 2$

Insulin

Isopropanol

$\mathrm{KCl}$

LB-agar

LB-medium

MEM vitamins $100 \mathrm{x}$

MEM amino acids 50x

$\mathrm{MgCl} 2 * 6 \mathrm{H} 2 \mathrm{O}$

$\mathrm{MgSO} 4 * 7 \mathrm{H} 2 \mathrm{O}$

$\mathrm{NaCl}$

NaEDTA

$\mathrm{NaHCO} 3$

$\mathrm{NaH} 2 \mathrm{PO} 4$

$\mathrm{NaH} 2 \mathrm{PO} 4 * \mathrm{H} 2 \mathrm{O}$

$\mathrm{NaOH}$

Page Ruler Prestained proteins (ladder)

Papaine

PBS (+/- Calcium Magnesium)

Phase lock gel heavy tube

phenol:chloroform:isoamyl
Ascent scientific

Roth

PAA

Roth

Roth

BioLog

Applichem

Flucka

Biochrom

Sigma

Bioline

Roth

Roth

Bioline

Fermentas

NEB

Roth

Sigma-aldrich

Merck

Merck

Roth

Biochrom

Roth

Biochrom

Biochrom

HyClone

Gibco

Sigma

Roth

Sigma

Roth

Roth

Gibco

Gibco

Merck or Sigma

Fluka or Merck

Sigma

Roth

Sigma

Sigma-Aldrich

Sigma

Roth

Fermentas

Worthinghton

Biochrom

5-Prime

AppliChem 
Phusion Polymerase

Picrotoxin

Polymerase T4 / Mango Taq

PonceauS

Porous membrane discs $(0.4 \mu \mathrm{m})$

PVDF membrane

2-propanol

Proteinase $\mathrm{K}$

QIAquick gel extraction kit

QIAquick PCR purification kit

Sodium Dodecyl Sulfate (SDS)

Sucrose

TEMED (N,N,N',N'-Tetramethylethylenediamine)

TRIS/HCl

Trypsin

Tween- 20
Finnzymes

Ascent Scientific

Bioline

Sigma-Aldrich

Millipore

Millipore

Roth

Roth

Qiagen

Qiagen

Roth

Roth

Uptima interchim

Roth

Biochrom

Roth

\section{Equipment}

\section{Instruments}

Borosilicate glass patch pipette

Camera \& its controller C2741-62

Double-barrel glass TST150-6

DNA electrophoresis chambers

Electroporator $\quad 2510$

Heating device (electrophysiology set-up)

Incubator $37^{\circ}$

Incubator $34^{\circ}$

ITC-18 data acquisition board

Odyssey scanmachine

Biosciences

Micromanipulator MP-225

MultiClamp700B amplifier

Nanoject II device

Osmometer Vapro 5520

pH meter PP-15

Puller P-97

Refrigerated Centrifuge 5417R

Table Centrifuge 5424R

Ultracentrifuge Beckman J2-MC

UV-photograph apparatus

Water system Arium 611VF

\section{Supplier}

King Precision Glass

Hamamatsu

World Precision Instr.

Harnischmacher

Eppendorf

npi

New Brunswick sc.

Labotech

InstruTECH/HEKA

Li-COR

Sutter Instrument

Axon instrument

Drummond Scientific

Wescor

Sartorius

Sutter Instrument

Eppendorf

Eppendorf

Beckman Instruments

Intas

Sartorius 
Software

IGOR Pro

WaveMetrics Inc.

\section{II.B. Methods}

Deionized water, from a Sartorius filter system was used to prepare all solutions.

\section{II.B.1. Molecular biology and biochemistry}

For the cloning of DNA constructs, standard methods for preparative and analytical digestion, ligation, and electroporation of Escherichia coli (E. coli)

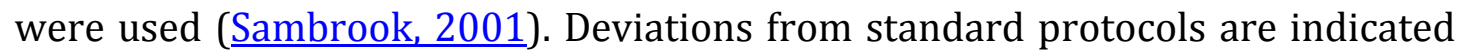
in the following sections.

All protocols were done according to the manufacturers' instruction unless stated.

\section{II.B.1.a. Electroporation of bacteria}

To $50 \mu \mathrm{l}$ of electrocompetent XL1-Blue or TOP10 bacteria (thawed on ice), $1 \mu \mathrm{l}$ of DNA ligation mixture was added and transferred in pre-cooled cuvette (Invitrogen). After electroporation of the plasmid using the electroporator 2510 device (1800V), $300 \mu$ l of LB medium without antibiotics was added to the bacteria and then plated on a LB plate containing the appropriate antibiotics (in this study, ampiciline). Plates were incubated at $37^{\circ} \mathrm{C}$ overnight in order for single bacteria colonies to grow separately.

\section{II.B.1.b. Plasmid preparation}

Single bacteria colony was picked up, inoculated in $5 \mathrm{ml}$ LB containing ampiciline and incubated overnight in a shaker at $37^{\circ} \mathrm{C}$. After a $7000 \mathrm{x}$ g centrifugation for 7 
minutes, DNA plasmid inside E.Coli was extracted using the P1, P2, P3 buffers from Qiagen, an alkaline lysis method (Doly, 1979). In order to remove the RNA, the samples were incubated $5 \mathrm{~min}$ at $55^{\circ} \mathrm{C}$. A test digest was performed in order to identify positives clones. When successful test digest pattern was observed on a agarose gel, a phenol/chloroform extraction was performed, by mixing the DNA containing solution with phenol:chloroform:isoamyl alcohol $(25: 24: 1)$ in a 1:1 ratio. The phases were separated by using heavy phase lock tube (Eppendorf), and centrifuged 1min at 14000rpm (rounds per minute). The supernatant was transferred and mixed with an equal volume of chloroform. After 1-minute centrifugation, the upper phase was collected and precipitated with 0.7 volume of isopropanol by $15 \mathrm{~min}$ centrifugation at $14000 \mathrm{rmp}$. The pellet was resuspended in $30 \mu$ l Elution Buffer (Qiagen).

\section{II.B.1.c.PCR screen}

Another way to identify positive clones (prior of the plasmid preparation) was to perform a PCR screen on numerous single colonies followed by plasmid isolation as described above.

The PCR screen was performed as followed:

Day 1:

Pick and spread single colony onto another LB plate with clear separation in between them. The plate was incubated overnight at $37^{\circ} \mathrm{C}$.

Day 2:

Single colony were picked and dipped in $0.2 \mathrm{ml}$ PCR tubes (8-tube strips) containing $10 \mu \mathrm{l}$ of water. $10 \mu \mathrm{l}$ of $2 \mathrm{x}$ master mix was then added, and samples proceeded into the PCR machine. For the PCR program selection, see section II.B.1.e example 1.

For a single reaction, the master mix was made of

$-6.4 \mu \mathrm{lddH} 20$

- $0.2 \mu$ l Mango Taq Polymerase 
- $0.2 \mu$ l forward primer $(50 \mathrm{pM})$

- $0.2 \mu$ l Reverse primer (50pM)

- $1 \mu \mathrm{l}$ dNTPs (2.5mM each)

- $2 \mu \mathrm{l} 10 \mathrm{x}$ TNK

\section{II.B.1.d. Ligation of DNA fragments}

Ligation of DNA fragments was performed by incubating the backbone of a plasmid with an appropriate amount of insert DNA with a ratio 1:3 (vector:insert) (Sambrook, 1989). A typical reaction consisted of $3 \mu \mathrm{l} 10 \mathrm{x}$ fermentas buffer, $1 \mu \mathrm{l}$ T4 DNA ligase and DNA fragments in a total volume of 30 $\mu$ l. The reaction was incubated at $16^{\circ} \mathrm{C}$ overnight in case of sticky ends ligation or $2 \mathrm{~h}$ at room temperature in case of blunt ends ligation. The mixture was then used directly for transformation.

\section{II.B.1.e. Polymerase Chain Reaction (PCR)}

The amplification of DNA fragments for cloning the different constructs used in this study was done with the high fidelity PFU polymerase or the Phusion

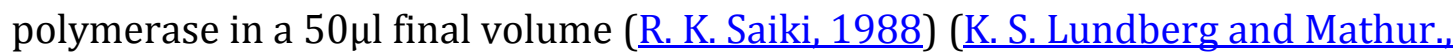
1991). The compounds of the reaction were added as followed:

$-10 \mu l 5 x$ reaction buffer

- $1 \mu$ l template DNA (e.g. 20ng plasmid DNA)

- $0.5 \mu$ l Forward primer (50 pmol)

- $0.5 \mu \mathrm{l}$ Reverse primer $(50 \mathrm{pmol})$

- $4 \mu \mathrm{l}$ dNTPs (2.5mM each)

- $1 \mu \mathrm{l} \mathrm{Taq} / \mathrm{Pfu}$ polymerase 
Using a PCR cycler, the following program was adjusted according to the melting/annealing temperature of the primers (a) and the size of the amplicon (c).

The melting temperature (Tm) of the primer was calculated according to the nearest neighbor thermodynamic theory using the following website: http://www.basic.northwestern.edu/biotools/oligocalc.html.

The annealing temperature of the primer was calculated by subtracting $\sim 5^{\circ} \mathrm{C}$ to the melting temperature.

Two types of programs were used depending on whether the PCR was aiming to only amplify a fragment of DNA (see example 1) or amplify and as well introduce a restriction site (see example 2). In the last case, a slightly different program was used where (a) was referring to the melting temperature of the short primers (not counting the additional restriction site) and (b) to the melting temperature of the long primers (full-length primer).

In every case, the polymerase was assumed to amplify 1000 base pairs per minute.

Example 1 (DNA amplification only):

- $\quad 98^{\circ} \mathrm{C} ; 2^{\prime}, 30 \mathrm{x}\left(98^{\circ} ; 30^{\prime \prime}, \mathrm{a}^{\circ} ; 45^{\prime \prime}, 72^{\circ} ; \mathrm{c}^{\prime}\right) 72^{\circ}, 10^{\prime} 4^{\circ}$

Example 2 (amplification and introduction of restriction site):

- $\quad 98^{\circ} \mathrm{C} ; 2^{\prime}, 15 \mathrm{x}\left(98^{\circ} ; 30^{\prime \prime}, \mathrm{a}^{\circ} ; 45^{\prime \prime}, 72^{\circ} ; \mathrm{c}^{\prime}\right) 25 \mathrm{x}\left(98^{\circ} ; 30^{\prime \prime}, \mathrm{b}^{\circ} ; 45^{\prime \prime}, 72^{\circ} ; \mathrm{c}^{\prime}\right)$ $72^{\circ}, 10^{\prime} 4^{\circ}$

\section{II.B.1.f. DNA gel electrophoresis}

DNA fragments were separated by horizontal electrophoresis in DNA electrophoresis chamber using agarose gels. Agarose gels were prepared by boiling $1 \%$ agarose $(\mathrm{w} / \mathrm{v})$ in $1 \mathrm{xTAE}$ buffer till all the agarose was dissolved. The 
solution was kept in $55^{\circ} \mathrm{C}$ water bath and supplemented with ethidium bromide. After pouring onto DNA gel trays and polymerized at room temperature, the gel was inserted in the running chamber and covered with 1xTAE buffer.

DNA samples were mixed with $6 \mathrm{x}$ loading sample buffer and pipetted into the gel pockets. The gel was run at constant voltage. Afterwards, the gel was documented using the Intas UV-light camera system.

50x TAE buffer:

242 Tris $\mathrm{g} / \mathrm{l}$

57.1 Acetic acid $\mathrm{ml} / \mathrm{l}$

100ml 0.5M EDTA (pH 8.0) g/l

\section{II.B.1.g. Extraction of DNA fragments from agarose gels}

Short illumination of ethidium bromide stained gels with UV-light renders possible to excise the appropriate DNA fragments from agarose gels.

The fragment was purified using the QIAquick gel extraction kit.

\section{II.B.1.h. Purification of DNA fragments after PCR}

Purification of DNA fragments directly after PCR was performed with the PCR Purification kit from Qiagen.

\section{II.B.1.i. Sequencing}

DNA sequencings were performed by the AGCT DNA Core Facility. For preparation, $1 \mu \mathrm{g}$ of DNA was diluted in $16 \mu$ d double distilled water along with the appropriate sequencing primers (50 pM). 


\section{II.B.1.j. Generation of the mutants}

The corresponding cDNA of the gene was cloned in frame into the vector pBluescriptII SK(-) (Stratagene) or FHUGW (대lüter et al., 2006). The PSD-95 cDNA sequence used in this study can be found under the accession number: NM_019621.1

\section{II.B.1.k. Pure knock down of endogenous PSD-95}

This construct was generated by Schlüter and colleagues ( $\underline{\text { Schlüter et al., 2006) }}$. The endogenous PSD-95 is knocked-down by the use of RNA interference technology. The short hairpin RNA (shRNA) against PSD-95 (sh95) targets the following sequence: GGA CAT CCA GGC ACA CAA G. It was cloned under the H1 promoter in the lentiviral transfer vector, FHUGW. GFP (Green Fluorescent Protein) is expressed under the Ubiquitin promoter and serves as a reporter thus allowing to visually identity cells of interest (expressing a mutant protein).

\section{II.B.1.l. Molecular replacement of endogenous PSD-95 by its mutant:}

\section{II.B.1.l.i. The Molecular replacement technique}

The molecular replacement was generated by Schlüter et al ( $\underline{\text { Schlüter et }}$ al., 2006). It finds its origin in the use of lentiviral vector systems to (over)express a protein of interest, and was implemented with the incorporation of a second promoter leading to the expression of a short hairpin RNA, conducting an efficient knockdown of the protein of interest. This approach also allows a temporally and spatially controlled manipulation, hence reducing any developmental compensation. Accordingly, it is thought to have minimal disturbance on the protein network, restricting the molecular manipulation to a particular set of cells under investigation. Moreover, in comparison to a 'classica' 
overexpression, molecular replacement does not rely on a dominant effect by the mutant, but rather on the restoration of a loss of function. Furthermore, the molecular replacement method can be combined with electrophysiological recordings, more specifically dual neighbor neuron whole-cell patch-clamping (one unmodified control cell and one GFP-fused recombinant protein-expressing infected cell). This allows for the stimulation of both neurons through a common pathway and comparisons between the electrophysiological properties of the two neurons.

The constructs used in this study were generated, where shRNA against PSD-95 is expressed under the $\mathrm{H} 1$ promoter and the mutant protein of interest under the Ubiquitin promoter. It should be noted that in order to not knockeddown the replacing (mutant) protein, a silent mutation was introduced into the recognition site of the shRNA thus rendering the shRNA able to only interfere with the endogenous protein.

Molecular replacement by full-length PSD-95 (sh95 + PSD-95).

This construct was kindly provided by Dr. Dr. Schlüter and is used as a control/reference.

In order to create molecular replacement vector expressing PSD-95 lacking certain domain(s) of interest, deletion of it/them was carried out basing on the same method:

Using the PSD-95 sequence contained in FHsh95pUPSD-95GW as a template, with different set of primers, different fragments of PSD-95 were amplified (according to the interest) while introducing restriction site at the $\mathrm{N}$-terminal part of the forward primer and the same at the C-terminal of the reverse primer, for example enzyme X.

The introduction of a restriction site was performed in order to facilitate the ligation.

After PCR, the DNA product was column purified and digested with enzymes of 
interest. Like X \& Y enzymes for the amplified N-terminal part and Y enzyme \& Z for the amplified C-terminal part. In the same time, the backbone FHsh95pUPSD95GW was also digested with $\mathrm{X}$ and $\mathrm{Z}$.

The three different products were run of an agarose gel (the percentage of the gel depending on the size of the band to isolate), then DNA band of interest was excised and purified.

The three fragments were then ligated in a 1:3:3 ratio.

Next the final construct was electroporated in electrocompetent cells and spread on agar plates.

Positives clones were finally isolated by mini plasmid preparation and sent for sequencing.

A summary of the different replacement constructs.

\begin{tabular}{|l|l|l|}
\hline $\begin{array}{l}\text { Name } \\
\text { of the } \\
\text { construct }\end{array}$ & $\begin{array}{l}\text { Deleted } \\
\text { Domain(s) }\end{array}$ & $\begin{array}{l}\text { Primers used } \\
\text { In small is the introduced sequence. } \\
\text { In small bold is the introduced restriction site. } \\
\text { In brackets, name of the enzyme recognizing } \\
\text { the restriction site. }\end{array}$ \\
\hline $\begin{array}{l}\text { Sh953 }+ \\
\text { SH3 domain }\end{array}$ & $\begin{array}{l}\text { PSD-95 5' fwd OS (EcoRI) } \\
\text { ggaattcGGCAGCCCTGAAGAACACATATGACG }\end{array}$ \\
& $\begin{array}{l}\text { PSD-95 5'SH3 rev (1/2 EcoRV) } \\
\text { atcGGGTTGCTTCGCAAGGATGCAGTC }\end{array}$ \\
& $\begin{array}{l}\text { PSD-95 3'SH3 fwd (1/2 EcoRV) } \\
\text { atcGTGGTCAAGGTTAAAGGCCAAGGAC }\end{array}$ \\
\hline
\end{tabular}




\begin{tabular}{|c|c|c|}
\hline & & $\begin{array}{l}\text { PSD-95 3' rev OS (EcoRI) } \\
\text { ggaattcAGATCTCTTCAAAGCTGTCGCCCTCTAC }\end{array}$ \\
\hline $\begin{array}{l}\text { Sh95 }+ \\
\Delta \text { GK }\end{array}$ & GK domain & Gift from Dr.Dr. Schlüter \\
\hline $\begin{array}{l}\text { Sh95 }+ \\
\Delta \text { PDZ3 }\end{array}$ & $\begin{array}{l}\text { PDZ3 } \\
\text { domain }\end{array}$ & $\begin{array}{l}\text { rP95 (XbaI) fwd_16682 } \\
\text { TCGTCTAGACCACCATGGACTGTCTCTGTATAGT } \\
\text { G } \\
\text { P95 P2 rev (HinDIII) } \\
\text { tgcaagcttgctagcCCGGGGAARGTCTTCCTCC } \\
\text { rP95 SH3 fwd (HinDIII) } \\
\text { tgcaagcttGGCTTCTACATTAGGGCCC } \\
\text { WRE2 rev_10324 } \\
\text { CATAGTTAAGAATACCAGTCAATC }\end{array}$ \\
\hline $\begin{array}{l}\text { Sh95 }+ \\
\text { PDZ12 }\end{array}$ & $\begin{array}{l}\text { PDZ3, SH3, } \\
\text { GK domains }\end{array}$ & Gift from Dr.Dr. Schlüter \\
\hline $\begin{array}{l}\text { Sh95 + } \\
\text { PDZ12-GK }\end{array}$ & $\begin{array}{l}\text { PDZ3, SH3 } \\
\text { domains }\end{array}$ & $\begin{array}{l}\text { p95fwd_16682 (Xbal) } \\
\text { TCGTCTAGACCACCATGGACTGTCTCTGTATAGT } \\
\text { G } \\
3 \text { rev PDZ2 (BstBI) } \\
\text { gcttcgaaCGAGGTTGTGATGTCTGGGGG } \\
5 \text { fwd GK (BstBI) } \\
\text { gcttcgaaTGGTCAAGGTTAAAGGCCAAGGACTG } \\
\text { EGFP rev_14952 (BsrG) }\end{array}$ \\
\hline
\end{tabular}




\begin{tabular}{|l|l|l|}
\hline & & cgtctcagatctTTACTTGTACAGCTCGTCCATGa \\
\hline
\end{tabular}

\section{II.B.1.l.ii. Overexpression vectors}

The over-expression vector is dimilar as the one used for the molecular replacement, apart from a missing sh95p sequence under the H1 promoter. For example, FHUPSD-95dGKGW, in short, OE $\triangle$ GK and FHUPSD-95-PDZ12-GKGW (OE PDZ12-GK).

Another set of replacement constructs did not knockdown PSD-95, but knockdown SAP102 and express PSD-95 mutants presented above. In this case, the shRNA against SAP102 was expressed under the U6 promoter. The FU6sh102UGW vector was kindly provided by Dr. Y.Liu in our lab, and was used as a backbone to clone between Xbal and BsrGI the mutant of interest.

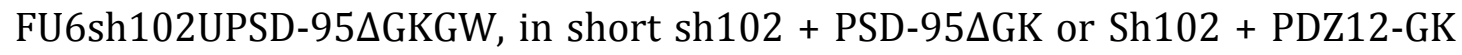
or Sh102 + PSD-95 SSH3.

\section{II.B.1.m. List of primers}

\begin{tabular}{|l|l|l|l|l|}
\hline Name of the primer & Sequence & $\begin{array}{l}\text { Long } \\
\text { primer } \\
\text { Tm }\end{array}$ & $\begin{array}{l}\text { Short } \\
\text { primer } \\
\text { Tm }\end{array}$ & From \\
\hline PSD-95 5' fwd OS & $\begin{array}{l}\text { ggaattcGGCAGCCCTGA } \\
\text { AGAACACATATGACG }\end{array}$ & $66^{\circ} \mathrm{C}$ & $61^{\circ} \mathrm{C}$ & Sigma \\
\hline $\begin{array}{l}\text { (EcoRI introduced) } \\
\text { (1/2 EcoRV introduced) }\end{array}$ & $\begin{array}{l}\text { atcGGGTTGCTTCGCAA } \\
\text { GGATGCAGTC }\end{array}$ & $64^{\circ} \mathrm{C}$ & $61^{\circ} \mathrm{C}$ & Sigma \\
\hline PSD-95 3'SH3 fwd & atcGTGGTCAAGGTTAA & $61^{\circ} \mathrm{C}$ & $57^{\circ} \mathrm{C}$ & Sigma \\
\hline
\end{tabular}




\begin{tabular}{|c|c|c|c|c|}
\hline (1/2 EcoRV introduced) & AGGCCAAGGAC & & & \\
\hline $\begin{array}{l}\text { PSD-95 3' rev OS } \\
\text { (EcoRI introduced) }\end{array}$ & $\begin{array}{l}\text { ggaattcAGATCTCTTCA } \\
\text { AAGCTGTCGCССTCTAC }\end{array}$ & $66^{\circ} \mathrm{C}$ & $63^{\circ} \mathrm{C}$ & Sigma \\
\hline rP95 XbaI fwd_16682 & $\begin{array}{l}\text { TCGTCTAGACCACCATG } \\
\text { GACTGTCTCTGTATAGT } \\
\text { G }\end{array}$ & $65^{\circ} \mathrm{C}$ & & MPI-em \\
\hline $\begin{array}{l}\text { P95 P2 rev } \\
\text { (HinDIII introduced) }\end{array}$ & $\begin{array}{l}\text { tgcaagcttgctagcCCGGG } \\
\text { GAARGTCTTCСTCC }\end{array}$ & $69^{\circ} \mathrm{C}$ & $51^{\circ} \mathrm{C}$ & Sigma \\
\hline $\begin{array}{l}\text { rP95 SH3 fwd } \\
\text { (HinDIII introduced) }\end{array}$ & $\begin{array}{l}\text { tgcaagcttGGCTTCTACA } \\
\text { TTAGGGCCC }\end{array}$ & $64^{\circ} \mathrm{C}$ & $51^{\circ} \mathrm{C}$ & Sigma \\
\hline WRE2 rev_10324 & $\begin{array}{l}\text { CATAGTTAAGAATACC } \\
\text { AGTCAATC }\end{array}$ & $49^{\circ} \mathrm{C}$ & & MPI-em \\
\hline p95fwd_16682 (XbaI) & $\begin{array}{l}\text { TCGTCTAGACCACCATG } \\
\text { GACTGTCTCTGTATAGT } \\
\text { G }\end{array}$ & 65 & 56 & Sigma \\
\hline $\begin{array}{l}3 \text { rev PDZ2 BstBI } \\
\text { (BstBI introduced) }\end{array}$ & $\begin{array}{l}\text { gcttcgaaCGAGGTTGTG } \\
\text { ATGTCTGGGGG }\end{array}$ & 65 & 62 & Sigma \\
\hline $\begin{array}{l}5 \text { fwd GK BstBI } \\
\text { (BstBI introduced) }\end{array}$ & $\begin{array}{l}\text { gcttcgaaTGGTCAAGGT } \\
\text { TAAAGGCCAAGGACTG }\end{array}$ & 65 & 59 & Sigma \\
\hline EGFP rev_14952 (BsrGI) & $\begin{array}{l}\text { cgtctcagatctTTACTTGT } \\
\text { ACAGCTCGTCCATGa }\end{array}$ & 66 & 54 & MPI-em \\
\hline
\end{tabular}




\section{II.B.1.n. SDS-PAGE \& Western Blot}

Protein samples were separated according to their molecular weight by SDSPolyacrylamid gel electrophoresis (SDS-PAGE, introduced by (‥ L. Shapiro, 1967) in a 4-20\% precast NuPage 4-12\% BisTris gel (Invitrogen) for up to 5 hours at $120 \mathrm{~V}$, dependent on the desired resolution and molecular weight of the protein of interest.

After assembly of the chamber, samples were loaded $(10 \mu$ in case of samples harvested from dissociated culture) as well as the ladder $6 \mu$ l.

The assembly was filled with $1 \mathrm{X}$ SDS running buffer and the gel was run at a constant voltage of $50 \mathrm{~V}$ for approximately 15 minutes and then at $150 \mathrm{~V}$ till the end of the run. The run was stopped when the bromophenol blue had reached the end of the gel. Gels were then subjected to Western blotting (Towbin et al. 1979).

Proteins were transferred after SDS-PAGE onto a protran nitrocellulose membrane using a Harnischmacher TRANSBLOT-apparatus.

A blotting sandwich was assembled in cold transfer buffer according the manufacturer's protocol. Proteins were transferred at $4^{\circ} \mathrm{C}$ in transfer buffer at constant voltage $(200 \mathrm{~mA}$ for $1 \mathrm{~h} 30)$. The pre-stained protein ladder marker, in addition to showing the molecular weight, was also a first indicator of a successful protein transfer. To visualize transferred samples the PonceauS staining was performed.

20x SDS running buffer:

1M MOPS, 1M Tris, 20mM EDTA, 2\% SDS.

Transfer buffer:

3g/l Tris, 14.4g/l Glycin, 20\% Methanol. 


\section{II.B.1.o. Immunodetection}

After successful electrophoretic transfer, membranes were removed from the sandwiches and blocked in 5\% milk-TBS-T for 30 minutes at room temperature, under gentle shaking. Incubation with an appropriate primary antibody diluted in 2.5\% milk-TBST, was performed either for 90 minutes at room temperature or overnight at $4{ }^{\circ} \mathrm{C}$. The antibody solution was removed and membranes were washed $3 \times 5$ minutes with TBS-T under vigorous shaking. The appropriate secondary antibody was applied, in a 1:10000 dilution into TBS-T for 90 minutes at room temperature under gentle shaking (light protected). The membrane was washed 3 times for 10 min with TBS-T under vigorous shaking. A final washing step was done in TBS (5 min). Immunofluorescent bands were visualized with the Odyssey Infrared Imaging System. The quantification was performed with the Odyssey analysis software.

TBS:

20mM Tris/HCL (pH 7.5), 140mM NaCl.

TBS-T:

TBS, 0.1\% Tween20.

\section{II.B.1.p. Antibodies}

\begin{tabular}{lll}
\hline \hline Antibody & Dilution & Supplier \\
\hline Primary antibodies & & \\
$\alpha$-Mortalin (mouse) & $1: 10000$ & NeuroMab \\
$\alpha$-PSD-93 (mouse ) & $1: 10000$ & NeuroMab \\
$\alpha$-PSD-95 (mouse) & $1: 10000$ & NeuroMab
\end{tabular}




$\begin{array}{lll}\alpha \text {-SAP-97 (mouse) } & 1: 2000 & \text { NeuroMab } \\ \alpha \text {-SAP-102 (mouse) } & 1: 2000 & \text { NeuroMab }\end{array}$

\section{Secondary antibodies}

$\begin{array}{lll}\alpha \text {-mouse Alexa Fluor } 680 \text { (goat) } & 1: 15000 & \text { Invitrogen } \\ \alpha \text {-rabbit Alexa Fluor } 680 \text { (goat) } & 1: 15000 & \text { Invitrogen } \\ \alpha \text {-mouse IR800 (goat) } & 1: 15000 & \text { Li-COR Biosciences } \\ \alpha \text {-rabbit IR800 (goat) } & 1: 15000 & \text { Li-COR Biosciences }\end{array}$

\section{II.B.1.q. Genotyping}

The PSD-95 KO mouse line used in this study was generated by Yao et al (Yao et al., 2004). The mice are housed in the institute's animal facility with a $12 \mathrm{~h}$ day light cycle. Each animal genotype was determined by PCR based on the protocol developed by the Jackson laboratory (Bar Harbor, ME, USA). At P2/P3 a 2mm mouse tail-piece was digested overnight in $200 \mu \mathrm{l}$ lysis buffer at $55^{\circ} \mathrm{C}$. Proteinase $\mathrm{K}$ was inactivated by boiling samples for 10 minutes. $2 \mu \mathrm{l}$ of lysate was used for genotyping PCR in a $20 \mu \mathrm{l}$ reaction using the oligonucleotides GKoptFor2 and GKoptrev2, and the Taq polymerase (see typical PCR reaction below) (Mullis K, Faloona F, Scharf S, Saiki R, Horn G, Erlich H. 1986. Cold Spring Harb Symp Quant Biol 51 Pt 1: 263-73). The following PCR program was used for both genotypes: $94^{\circ} \mathrm{C} 5 \mathrm{~min}$, repeat 35 times $\left(94^{\circ} \mathrm{C}\right.$ for $45 \mathrm{sec}, 55^{\circ} \mathrm{C}$ for $45 \mathrm{sec}, 72^{\circ} \mathrm{C}$ for $\left.1 \mathrm{~min}\right)$, followed by $72^{\circ} \mathrm{C}$ for $10 \mathrm{~min}$ then holding at $4^{\circ} \mathrm{C}$. PCR products were analyzed on $1 \%$ sodium tetraborate gels containing ethidium bromide, visualized by exposure to ultraviolet light (Southern E. 1979. Methods Enzymol 68: 152-76). A band at $255 \mathrm{bp}$ shows the presence of the wild type allele while a band at $355 \mathrm{bp}$ stands for the KO allele.

Typical PCR reaction:

- $\quad 2 \mu \mathrm{l}$ sample

- $\quad 2 \mu \mathrm{l}$ TNK buffer (10x) 
- $\quad 0.2 \mu \mathrm{l}$ Primer $(50 \mathrm{pmol} / \mu \mathrm{l})$

- $\quad 0.2 \mu \mathrm{l}$ Primer $(50 \mathrm{pmol} / \mu \mathrm{l})$

- $2 \mu \mathrm{l}$ dNTPS (2.5mM each)

- $\quad 0.2 \mu$ l Mango Taq Polymerase

- $\quad 13.4 \mu \mathrm{lddH} 20$

Lysis buffer:

PBND freshly complemented with $1.2 \mathrm{mg} / \mathrm{ml}$ proteinase $\mathrm{K}$

PBND:

$10 \mathrm{mM}$ Tris/HCl (pH 8.3), 50mM KCl, 2.5mM MgCl2-6H2O, 10\% gelatin, $0.45 \%$ Nonident P-40, 0.45\% Tween20.

TNK 10x:

$100 \mathrm{mM}$ Tris, $15 \mathrm{mM} \mathrm{MgCl} 2,500 \mathrm{mM} \mathrm{KCl}, 50 \mathrm{mM}$ NH4Cl, $\mathrm{pH} 8.5$

Sodium tetraborate $20 \mathrm{x}$ :

$0.1 \mathrm{M}$ sodium tetraborate $6 \mathrm{H} 20$

GKoptFor2

CAGGTGCTGCTGGAAGAAGG

GKoptrev2

CTACCCTGTGATCCAGAGCTG

\section{II.C.Cell culture techniques}

All cell culture plates were obtained from Greiner Bio-One. 


\section{II.C.1.a.Large scale lentivirus preparation}

In this purpose HEK293T cells were used (Graham et al. 1977). They were maintained in $10 \mathrm{~cm}$ cell culture dishes containing HEK medium (see below) and kept in a $37^{\circ} \mathrm{C}$ humidified incubator with $5 \% \mathrm{CO}$. The cells were passaged 1:51:20 every two to three days using standard procedures of trypsin-mediated dissociation of confluent monolayer cultures (Masters and Stacey 2007).

HEK medium:

High-glucose Dulbecco's Modified Eagle's Medium (Biochrom), 2mM Glutamax (Invitrogen), 10\% fetal calf serum (Biochrom).

For the production of lentivirus particles, the following components were cotransfected together into a 70-80\% confluent HEK293T cells in T-75 culture flasks (for transfection, see section ??)

- $\quad 10 \mu \mathrm{g}$ of transfer vector

- $\quad 7.5 \mu \mathrm{g}$ HIV-1 packaging vector delta8.9

- $\quad 2.5 \mu \mathrm{g}$ vsvg envelope glycoprotein vector

24 hours after the transfection, 10mM Hepes was added to the HEK293T culture dish and afterwards transferred to the same type of incubator but at $32^{\circ} \mathrm{C}$ temperature. Then the medium was collected 48 hours after the transfection and centrifuged for $5 \mathrm{~min}$ at $2000 \mathrm{~g}$. The supernatant was filtered through a $0.45 \mu \mathrm{m}$ PVDF membrane, and then viral particles were concentrated by a $90 \mathrm{~min} 36500 \mathrm{~g}$ centrifugation. The pellet was dissolved in $100 \mu$ l virus storage buffer (VSB) at $4^{\circ} \mathrm{C}$ overnight. Finally, the virus was aliquoted and stored in $-80^{\circ} \mathrm{C}$ freezer.

VSB:

20mM Tris-HCl (pH 8.0), 250mM NaCl, 5\% Sorbitol.

\section{II.C.1.b. Transfection}


TransIt 293 (Mirus Bio) transfection reagents were used.

\section{II.C.1.c. Hippocampal dissociated culture}

Dissociated hippocampal neuronal cultures were prepared from (P0-P1) Wistar rats of either sex based on (Baughman, 1986). After decapitation, the hippocampi were extracted in ice-cold dissection solution and transferred to the enzymatic solution (containing papain and DNasel) for $30 \mathrm{~min}$ digestion at $37^{\circ} \mathrm{C}$ on a rotating platform, according to the method of Huettner and Baughman (1986). Following the digestion, hippocampi were allowed to settle down in the tube, the enzymatic solution was replaced by the inactivation solution, which contains serum medium, DNaseI and BSA to dilute out the effect of any remaining papain. The inactivation solution was replaced with one hub of serum media and triturated with a flame-polished glass pipette with 15-20 strokes and centrifuged at $700 \mathrm{~g}$ for 5 minutes. The pellet of triturates cells was dissolved in 1 $\mathrm{ml}$ of 5\% serum medium. Around 100000 cells were plated per well of a poly-Dlysine-coated 24 well plate into $1 \mathrm{ml}$ of culture medium and kept at $37^{\circ} \mathrm{C}$ incubator with 5\% CO2 concentration. After 4 days in vitro (DIV 4), astrocyte growth was inhibited with FUDR (Sigma-Aldrich). On DIV7, half of the medium was exchanged with fresh culture media lacking Glutamate and Aspartate amino acids. The feeding was repeated every $4 / 5$ days.

Dissection solution (mGBSS) :

1.5mM CaCl2*2H2O, 4.9mM KCl, 0.2mM NaH2PO4*H2O, 11mM MgCl2*6H20, 0.3mM MgSO4*7H20, 130mM NaCl, 2.7mM NaHCO3, 0.8mM Na2HPO4, 22mM HEPES, 5mM Glucose*H2O

Enzymatic solution:

$10 \mathrm{ml}$ dissection solution, $11.39 \mathrm{mM}$ L-cysteine, 50mM NaEDTA (pH=8), 100mM $\mathrm{CaCl} 2,1 \mathrm{~N} \mathrm{NaOH}, 80 \mu \mathrm{l}$ Papaine, $100 \mu \mathrm{l}$ DNaseI. 
Inactivation Solution:

$25 \mathrm{mg}$ BSA, $10 \mathrm{ml}$ 5\% serum medium, $100 \mu \mathrm{l} 10 \mathrm{mg} / \mathrm{ml}$ DNAseI.

Culture medium:

DMEM/HamsF12 supplemented with 2\% B27, 1\% Glutamax, Glutamate and Aspartate amino acids

\section{II.C.1.d. Infection of dissociated culture}

On DIV7, after $50 \%$ of the media was exchanged, neurons were infected with $2 \mu \mathrm{l}$ of large-scale lentivirus preparation.

\section{II.C.1.e. Harvesting dissociated culture for western blotting}

During the harvesting procedure, the 24 wells plate was kept on ice packet. First, dissociated culture was washed once with PBS containing Calcium/Magnesium and then covered with $30 \mu \mathrm{l}$ of $2 \mathrm{X}$ SDS-sample buffer containing DTT $(27 \mathrm{mg} / 1 \mathrm{ml})$. Cells were scratched from the bottom of each well, transferred to eppendorf and boiled for 5 minutes.

4X SDS sample buffer:

0.8M Tris/HCl (pH 8.4), 40\% (w/v) glycerol, 8\% SDS, 2mM EDTA, $0.075 \%$ bromphenolblue. 13mM dithiothreitol was added before use.

\section{II.C.1.f. Hippocampal organotypic slices}

Organotypic hippocampal slices were prepared from postnatal day 7-8 Wistar rat or PSD-95 KO mice of both sexes according to the Stoppini method (Stoppini et al., 1991) with some modifications stated here. Animals were anesthetized 
with isoflurane and then decapitated. The skull was washed with sucrose cutting buffer. The procedure was repeated after exposing the brain. Once extracted the whole brain was immerged in frozen/liquid interface sucrose cutting buffer. Hippocampi of both hemispheres were dissected out and meninge partially removed. Transversal $300 \mu \mathrm{m}$ thickness slices were cut using a home made guillotine, then placed back into liquid sucrose cutting buffer in order to separate them. Next, they were transferred into oxygenated recovery ACSF for 30minutes at room temperature. Once this step was done, they were washed into cold BME supplemented with magnesium chloride (in order to limit firing of the slice and thus excitotoxicity) and then plated onto a square membrane placed on the insert (porous membrane discs) making the interface with the feeding media contained in a $2 \mathrm{~cm}$ dish. The slices were kept at $37^{\circ} \mathrm{C}$ during the first day of culture and transferred to $34^{\circ} \mathrm{C}$ incubator for the remaining time.

Sucrose cutting buffer:

$204 \mathrm{mM}$ Sucrose, $26 \mathrm{mM}$ NaHCO3, $10 \mathrm{mM}$ d-Glucose, $2.5 \mathrm{mM} \mathrm{KCl}, 1 \mathrm{mM}$ NaH2PO4*H2O, 4mM MgSO4*7H2O, 1mM CaCl2*2H2O, 4mM l- Ascorbic acid; sterile filtered.

Recovery ACSF (artificial cerebrospinal fluid): $119 \mathrm{mM} \mathrm{NaCl}, 26 \mathrm{mM}$ NaHCO3, 20mM d-Glucose, 2.5mM KCl, 1mM NaH2PO4, $4 \mathrm{mM} \mathrm{MgSO} 4 * 7 \mathrm{H} 2 \mathrm{O}, 4 \mathrm{mM} \mathrm{CaCl} 2 * 2 \mathrm{H} 20$. Sterile filtered and oxygenated for $30 \mathrm{~min}$ with $95 \%$ 02/5\% CO2 before use.

\section{II.C.1.g.Virus injection into CA1 hippocampal organotypic slice}

At DIV1 or DIV2, large scale produced viral particles were injected into one or two sites in the organotypic slice at the CA1 pyramidal cell layer, useing the Nanoject II device with the supplied capillary tips, and a custom-built micromanipulator. The amount of injected virus depended on the virus titer, 
thus the procedure was optimized to obtain a transduction of single cells in CA1.

\section{II.C.1.h. Feeding schedule for organotypic slice}

During the plating (day 0), slices were fed with HK20 medium, the next day the medium was renewed (HK20). Following injection (DIV1 or DIV2) the medium was again changed (HK20). Three days later it was substituted with HK5. At DIV4, electrophysiological recording could take place. Every other day, HK5 was renewed.

HK20:

49\% BME, 25\% EBSS, 25mM HEPES, 20\% Horse Serum (heat-inactivated for $30 \mathrm{~min}$ at $55^{\circ} \mathrm{C}$, HyClone or Biochrom), $28 \mathrm{mM}$ d-Glucose, $1 \mathrm{mM}$ Glutamax, $88 \mu \mathrm{g} / \mathrm{ml} \mathrm{l-Ascorbic} \mathrm{acid,} 1 \mu \mathrm{g} / \mathrm{ml}$ Insulin, 0.25\% 100X MEM-Vitamins, 0.49\% 50X MEM-amino acids.

HK5:

63.3\% BME, 25\% EBSS, 25mM HEPES, 5\% Horse Serum (heat-inactivated for $30 \mathrm{~min}$ at $55^{\circ} \mathrm{C}$, HyClone or Biochrom), $28 \mathrm{mM}$ d-Glucose, $2 \mathrm{mM}$ Glutamax, $88 \mu \mathrm{g} / \mathrm{ml} \mathrm{l-Ascorbic} \mathrm{acid,} 1 \mu \mathrm{g} / \mathrm{ml}$ Insulin, 0.32\% 100X MEM-Vitamins, 0.63\% 50X MEM-amino acids.

EBSS:

1.8mM CaCl2*2H2O, $1 \mathrm{mM} \mathrm{NaH} 2 \mathrm{PO}^{*} * \mathrm{H} 2 \mathrm{O}, 0.8 \mathrm{mM} \mathrm{MgSO} 4 * 7 \mathrm{H} 2 \mathrm{O}, 116 \mathrm{mM} \mathrm{NaCl}$, 26.2mM NaHCO3, 5.4mM KCl, 5mM d-Glucose*H2O.

\section{II.D. Electrophysiology}




\section{II.D.1. Data Acquisition}

The slice cultures used in the experiments were maintained 5 to 10 days in vitro after the preparation at $34^{\circ} \mathrm{C}$, and used during this time interval for electrophysiological recordings.

For the experiments, organotypic slices were transferred into a heated submerged recording chamber $\left(\sim 30^{\circ} \mathrm{C}\right)$, where they were continuously perfused (2-3ml/min) with carbogenated (95 \% 02, $5 \%$ CO2) ACSF. Schaeffer collaterals were stimulated extracellularly by brief $(0.2 \mathrm{~ms})$ current pulses (once every 5 seconds) from a bipolar electrode filled with recording ACSF. Stimulus strength ranged between $\mathrm{Y}$ and $\mathrm{X} \mu \mathrm{A}$. Somatic whole-cell voltage-clamp recordings were made from visually identified CA1 pyramidal cells (under infrared differential interference contrast microscope) using a 2-4 MOhm glass electrode filled with internal recording solution. Moreover, dual patch of an infected green cell and its neighboring control cell was performed in order to to compare their electrophysiological properties. For each pair of cells a minimum of 40 sweeps was collected using a patch clamp amplifier, an A/D converter and customprogrammed acquisition software (Igor pro). Series and input resistance was monitored by a $5 \mathrm{mV}$ hyperpolarizing step. Cells in which series resistance varied by $25 \%$ or was higher than $20 \mathrm{M} \Omega$ during a recording session were discarded. AMPA and NMDA excitatory postsynaptic currents (EPSCs) were obtained by evoking dual component responses while voltage-clamping neurons at $-60 \mathrm{mV}$ and $+40 \mathrm{mV}$ respectively. The different components of the EPSC were differentiated based on the difference in AMPA and NMDA receptors' decay kinetics. AMPARs decay within few milliseconds, whereas NMDARs decay over more than 100 of milliseconds. Therefore the NMDAR contribution was determined by measuring the EPSC peak amplitude $60 \mathrm{~ms}$ after the onset of the EPSC, a time point where the AMPAR component has essentially decayed to zero. The AMPAR component of EPSCs was measured as the peak amplitude obtained at a holding potential of $-60 \mathrm{mV}$ (Figure 8). 
A.

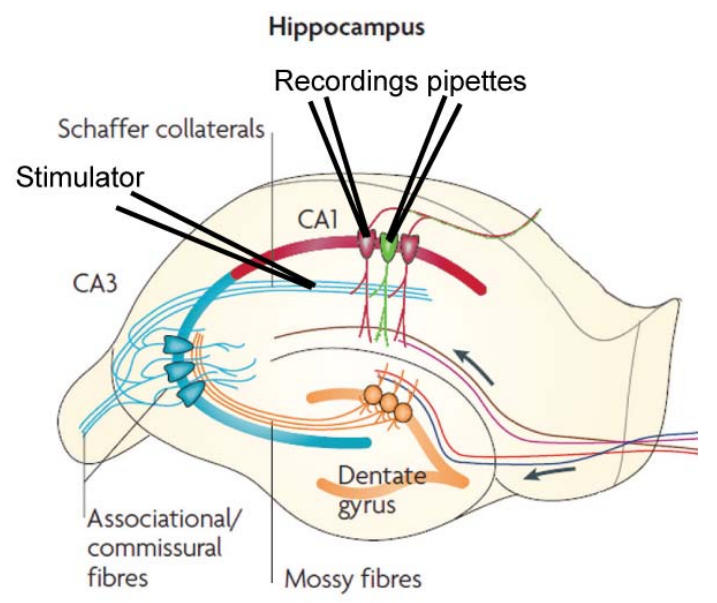

B.

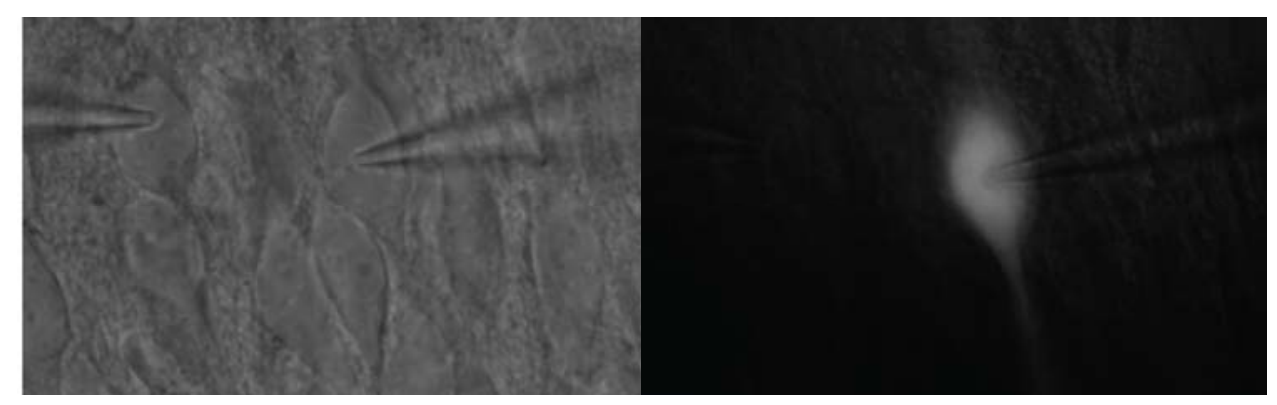

C.

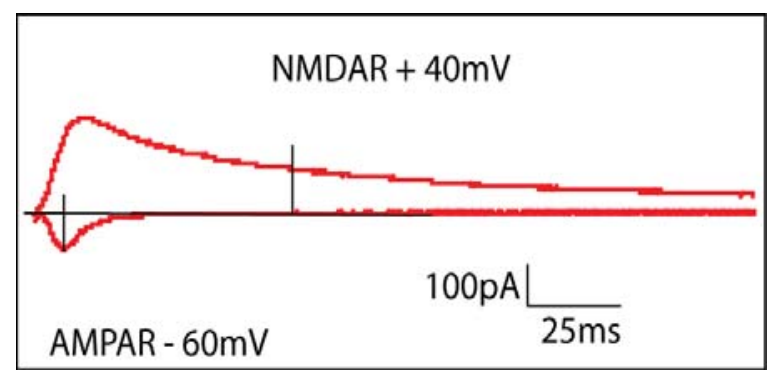

Figure 8. Simultaneous dual whole-cell patch-clamp recordings of hippocampal organotypic slice cultures.

Lentivirus was used to infect single cells in the CA1 hippocampal region (indicated in green) which could be visualized with fluorescent light sources (B). Recordings were 
performed by voltage-clamping an infected and a neighboring control cell while stimulating the Schaeffer collateral pathway (Figure 8C adapted from (Neves et al., 2008). . The cells were voltage-clamped at $-60 \mathrm{mV}$ to record AMPAR EPSCs. NMDAR EPSCs were approximated by recording at $+40 \mathrm{mV}, 60 \mathrm{msec}$ after the peak, when AMPARmediated currents have largely decayed (C).

Recording ACSF (artificial cerebrospinal fluid):

$119 \mathrm{mM} \mathrm{NaCl}, 26 \mathrm{mM}$ NaHCO3, 20mM d-Glucose, 4mM MgSO4*7H20, 2.5mM KCl, $1 \mathrm{mM}$ NaH2PO4, 4mM CaCl2*2H2O. The ACSF was oxygenated for $30 \mathrm{~min}$ with

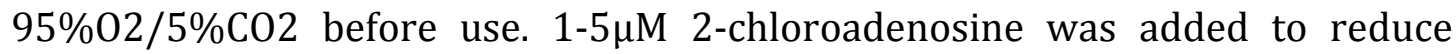
polysynaptic activity, and $50 \mu \mathrm{M}$ picrotoxin to block inhibitory transmission and isolate excitatory EPSCs.

Intracellular solution for patch pipettes:

117.5mM MeSO3H, 10mM HEPES, 17.75mM CsCl, 10mM TEA-Cl, 0.25mM EGTA, 10mM d-Glucose, 2mM MgCl2*6H2O, 4mM Na2ATP, 0.3mM NaGTP. CsOH 50\% $(\mathrm{w} / \mathrm{v})$ was used to adjust $\mathrm{pH}$ to 7.3 , and the solution was cooled to $4^{\circ} \mathrm{C}$ before adding Na2ATP and NaGTP. The osmolarity was adjusted to $290 \mathrm{mOsm}$ with CsCl. The solution was filtered with a $0.2 \mu \mathrm{m}$ syringe filter, aliquoted and stored at $80^{\circ} \mathrm{C}$ for up to 3 months.

\section{II.D.2. Off line Analysis}

All physiology data were analyzed with in-house Igor Pro software.

The different components of the EPSC were decomposed based on the difference in AMPA and NMDA receptors' decay kinetics. Hence, AMPARs decay within few milliseconds, whereas NMDARs decay over 100's of milliseconds. Therefore the NMDAR contribution was determined by measuring the EPSC peak amplitude $\underline{60}$ ms after the onset of the EPSC, a time point where the AMPAR component has essentially decayed to zero. 
All data are expressed as mean +/- standard error of the mean (SEM) that is shown as error bars in the presented graphs. Statistical significance of log transformed data was determined using two-tail paired student $t$ tests for between control and infected cell's comparisons and two-tail unpaired tests for between-group comparisons. A p value equal to, or smaller than 0.05 was considered statistically significant. Sample size $\mathrm{n}$ refers to the number of cells that were analyzed.

\section{Results}

The aim of this study was to understand how changes in synaptic strength correlate with changes in the molecular composition of synapses. PSD-95, one of the four members of the DLG-MAGUK family, has been shown to play a central role in regulating basal synaptic transmission (El-Husseini et al., 2000b) ( $\underline{\text { Schnell }}$ et al., 2002) (Ehrlich, 2004) (대lüter et al., 2006) (Elias et al., 2006) (Carlisle et al., 2008). PSD-95 is a modular protein composed of distinct domains. Starting from the N-terminus, PSD-95 contains three PDZ domains, followed by an SH3 domain, and a catalytically inactive GK domain (Kim and Sheng, 2004).

To determine which properties of PSD-95 are important for AMPAR delivery to synapse, I generated mutant and truncated forms of PSD-95. I analyzed them in 
the molecular replacement context, which consists of knocking-down the endogenous protein and simultaneously replacing it with one mutant of interest (Schlüter et al., 2006). The molecular replacement technique is advantageous because it can be used within specific time and space restrictions, and it is based on restoring of a loss of function. In addition, the endogenous level of PSD-95 was shown to be a critical parameter influencing the outcome of the results and thus their interpretations ( $\underline{\mathrm{Xu} \text { et } \mathrm{al} ., 2008)}$. That's why, for the purpose of this study, I used the molecular replacement method to manipulate a limited number of single mature neurons among an intact network of cells, in combination with electrophysiological recordings. I took advantage of organotypic hippocampal slice cultures in conjunction with lentiviral gene transfer to express GFP-tagged (mutant) proteins in CA1 hippocampal pyramidal cells. All electrophysiological experiments involved simultaneous dual whole-cell patch-clamp recordings from a lentiviral-infected cell and a neighboring uninfected cell. The amplitude of synaptic AMPAR currents was recorded at $-60 \mathrm{mV}$ and NMDAR EPSCs were recorded at $+40 \mathrm{mV}$, and were measured at a latency where AMPAR EPSCs had already decayed to baseline. The relative amplitudes of EPSC responses evoked by activating the same presynaptic afferents (Schaeffer collaterals) were directly compared, allowing the effects of the recombinant protein in the postsynaptic neuron on synaptic currents to be assessed.

The strategy adopted here to test the requirements for specific domains of PSD95 with respect to synaptic transmission was to delete them from the protein and assess the effect of the mutant protein on AMPAR and NMDAR transmissions when endogenous PSD-95 was knocked-down or absent (PSD-95 KO).

The aim of the study was to more precisely define which domain(s) of the Cterminal part of PSD-95 is/are required in conjunction with the $\mathrm{N}$-terminus and the two first PDZ domains for regulating basal synaptic transmission at the hippocampal CA3-CA1 connection. 


\section{III.A. Molecular replacement studies in rat hippocampal organotypic slice cultures}

\section{III.A.1. shRNA mediated knock-down of PSD-95 in hippocampal dissociated culture}

Before starting electrophysiological recordings, the first step was to test the efficacy of the shRNA that knocked down endogenous PSD-95 (named sh95) in rat hippocampal dissociated cultures. This experiment was done in such a way as to allow for a high percentage of neurons to be transduced by lentiviral particles, which would thus express the shRNA against PSD-95. This permits a good assessment of the knockdown effect.

This experiment was performed on P0 in dissociated rat hippocampal neuronal cultures infected at DIV7 with the lentivirus expressing the shRNA against PSD95.This was followed by a western-blot probe of the harvested samples. Neurons were examined 7days after virus infection (DIV 14). The blot showed that overall PSD-95 expression was reduced by in average 90\% compared to controls (Figure 9). 


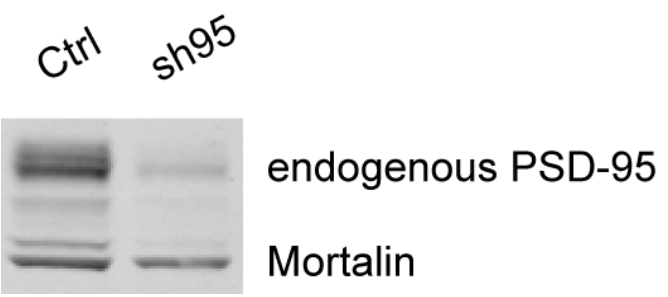

Figure 9. Acute knockdown, using short hairpin RNA against PSD-95 (sh95) delivered by lentivirus.

sh95 resulted in an almost complete loss of endogenous PSD-95 in dissociated rat hippocampal neuronal cultures, probed by western-blot. For all western-blot figures, mortalin is used to normalize the signal to the amount of protein per lane.

\section{III.A.2. Effect of PSD-95 knock-down on basal synaptic transmission}

Next, the absence of PSD-95 was evaluated at the electrophysiological level, using rat organotypic slice cultures. Recordings from a number of pairs are presented in a scatter plot where the X-axis represents the EPSC amplitude of control cells, and the Y-axis represents the EPSC amplitude of infected neurons (Figure 10). The graph is separated in the middle by a diagonal line, indicating equal responses between control and infected cell. In the case of the PSD-95 knockdown, the majority of the dots representing pair recordings of AMPAREPSC amplitude, including the mean (red square), are below the diagonal line (Figure 10). This indicates a reduction in the amplitude of AMPAR-EPSCs. Indeed, the lack of PSD-95 resulted in an approximately 50\% decrease in AMPAR-EPSC amplitude, while NMDAR-EPSC amplitude remained the same, as previously shown (Elias et al., 2006) (Schlüter et al., 2006). 
A.

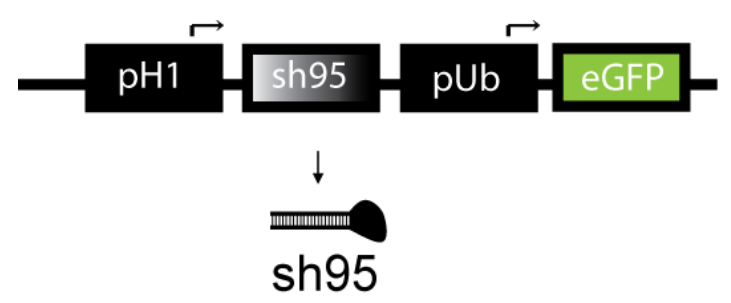

B. 

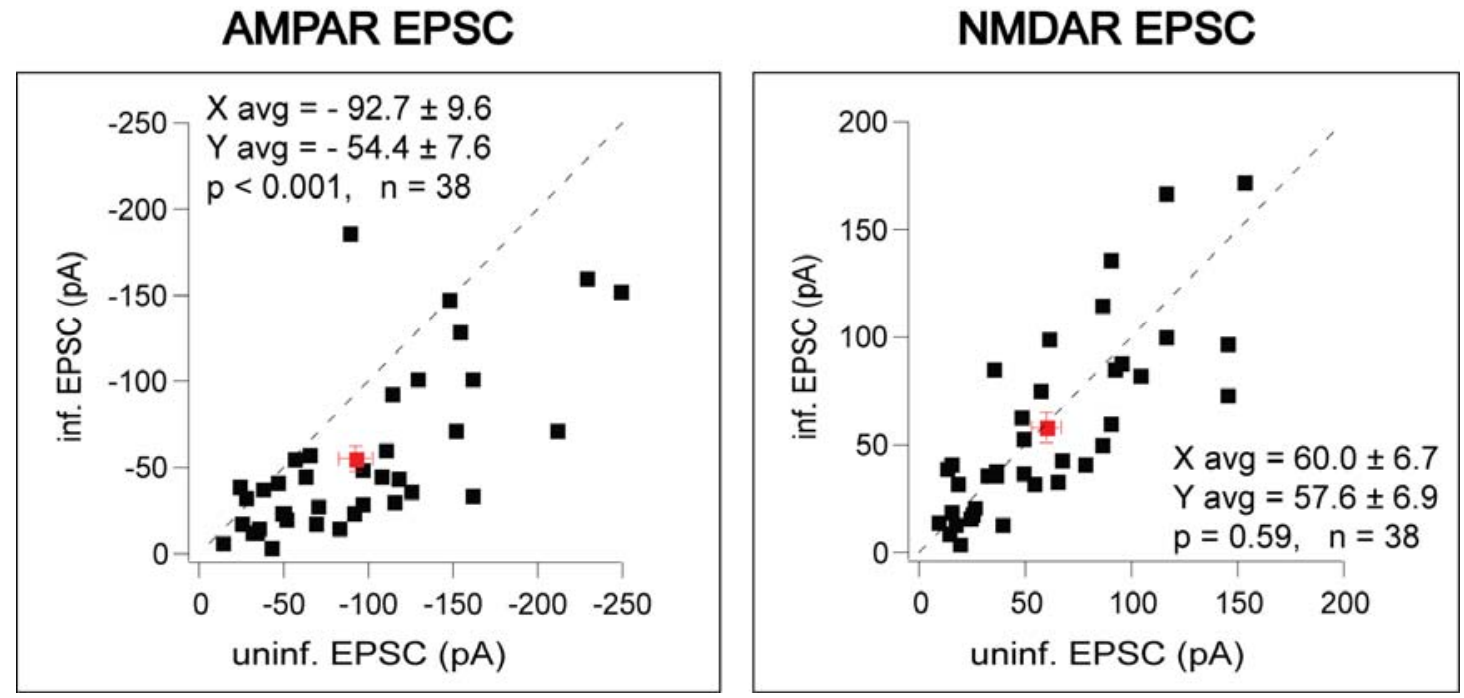

Figure 10. Effects of PSD-95 knockdown in basal synaptic transmission.

A) Illustration depicting shRNA mediated knockdown of PSD-95. B) Evoked EPSCs recorded simultaneously from an untransfected control neuron and a neuron expressing shRNA against PSD-95. For all EPSC scatter plots in this and subsequent figures, black and red squares represent amplitudes for individual pairs and mean \pm SEM, respectively. Distributions show a significant decrease in AMPAR-EPSC amplitudes (left) but not in NMDAR-EPSC amplitudes (right).

Our experiments required us to reduce background endogenous PSD-95 to prevent any confounding influence observed by the presence of endogenous protein, which could occur as the result of multimerization between mutant and endogenous protein. To this end, the molecular replacement technique was used to knockdown endogenous PSD-95 and simultaneously express one of its mutant forms.

\section{III.A.3. Molecular replacement by full-length PSD-95}

In order to evaluate whether the knockdown effect could be rescued by the expression of wild-type PSD-95, the molecular replacement technique was used to replace endogenous knocked-down PSD-95, with full-length PSD-95. Indeed, not only did it rescue the knockdown effect to the control cell level, the 
full-length PSD-95 replacement yielded a 2.3 fold increase in AMPAR-evoked currents (consistent with the previous study from (Schlüter et al., 2006)) along with a small, but significant, increase in NMDAR-evoked currents (Figure 11). Western blot data showed an efficient knockdown of endogenous PSD-95 concomitant to a high expression level of PSD-95 (an average of three times more intense than the endogenous PSD-95 band) (Figure 12).These results indicated that the molecular replacement technique used in this experiment is working properly which allowed me to use it as a trustable tool to investigate further the special requirements of PSD-95 domains in basal synaptic transmission.

A.

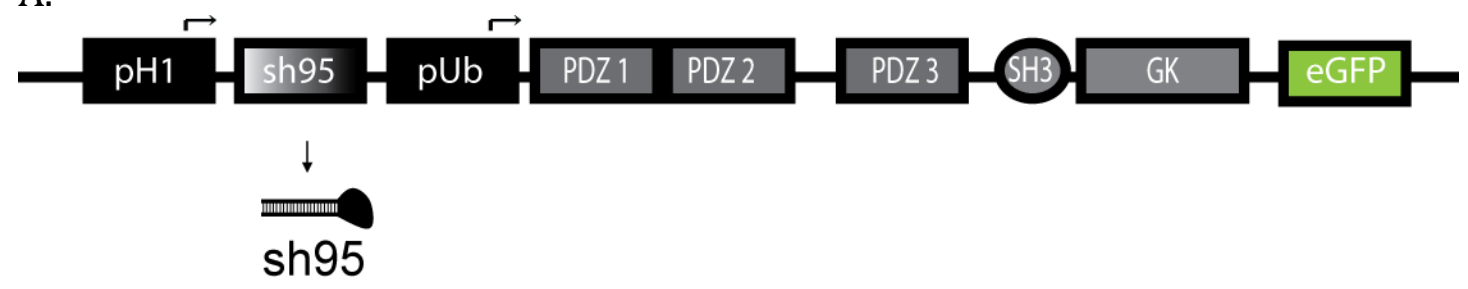

B. 
AMPAR EPSC

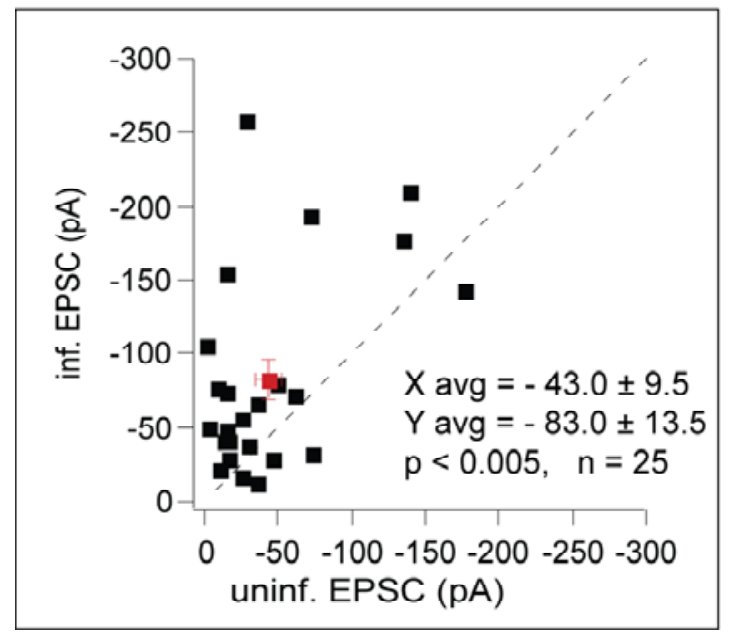

NMDAR EPSC

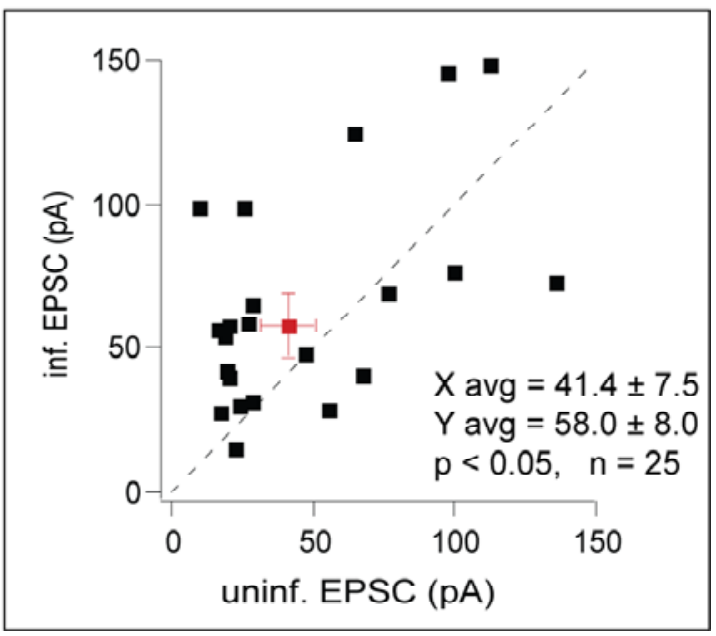

Figure 11. Effects of PSD-95 molecular replacement in basal synaptic transmission.

A) Illustration depicting the full-length PSD-95molecular replacement vector. B) Dual-whole cell evoked-EPSCs recorded from an untransfected control neuron and a PSD-95 knockdown simultaneously replaced with wild-type PSD-95 overexpressing neighbor. Distributions show a significant increase in AMPAR- EPSC amplitudes (left) concomitant with an increase in NMDAREPSC amplitudes (right).

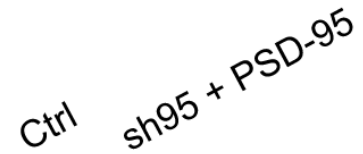<smiles>C=C</smiles>

PSD-95-GFP

endogenous PSD-95

Mortalin

Figure 12. Molecular replacement by wild type PSD-95 in rat hippocampal dissociated cultures assessed by western-blot.

A decrease in the endogenous level of PSD-95 and a robust expression of GFP-fused PSD-95 can be observed.

\section{III.A.4. Molecular replacement by PSD-95 expressing PDZ12 domains}

The molecular replacement of a truncated form of PSD-95 expressing PDZ12 domains was performed to assess if the expression of these two domains of PSD-95 are sufficient to rescue the previously seen effect of the knockdown. 
This experiment led to a $\sim 45 \%$ decrease in AMPAR-evoked currents with no change concerning the NMDAR component (Figure 13). This demonstrates that PSD-95 PDZ12 is not able to rescue the knockdown effect mediated by the shRNA against PSD-95, and that this truncated form of PSD-95 is non-functional (in the absence of endogenous PSD-95). Moreover, western blot data showed an efficient knockdown of endogenous PSD-95 concomitant to a high expression level of PSD-95 PDZ12 (Figure 14). This indicates that the observed decrease in synaptic transmission is not related to poor expression levels of the mutant form. By this, I could confirm already published results ( $\underline{\text { Schlüter et al., 2006) }}$.

A. 


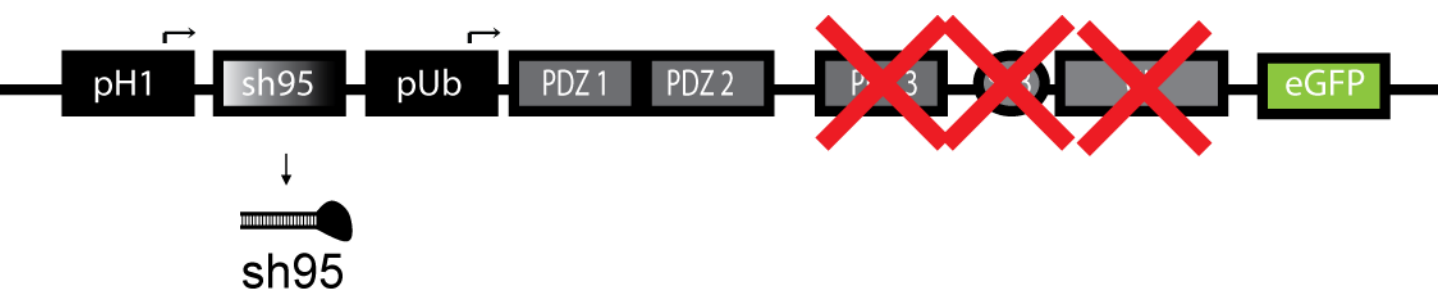

B.
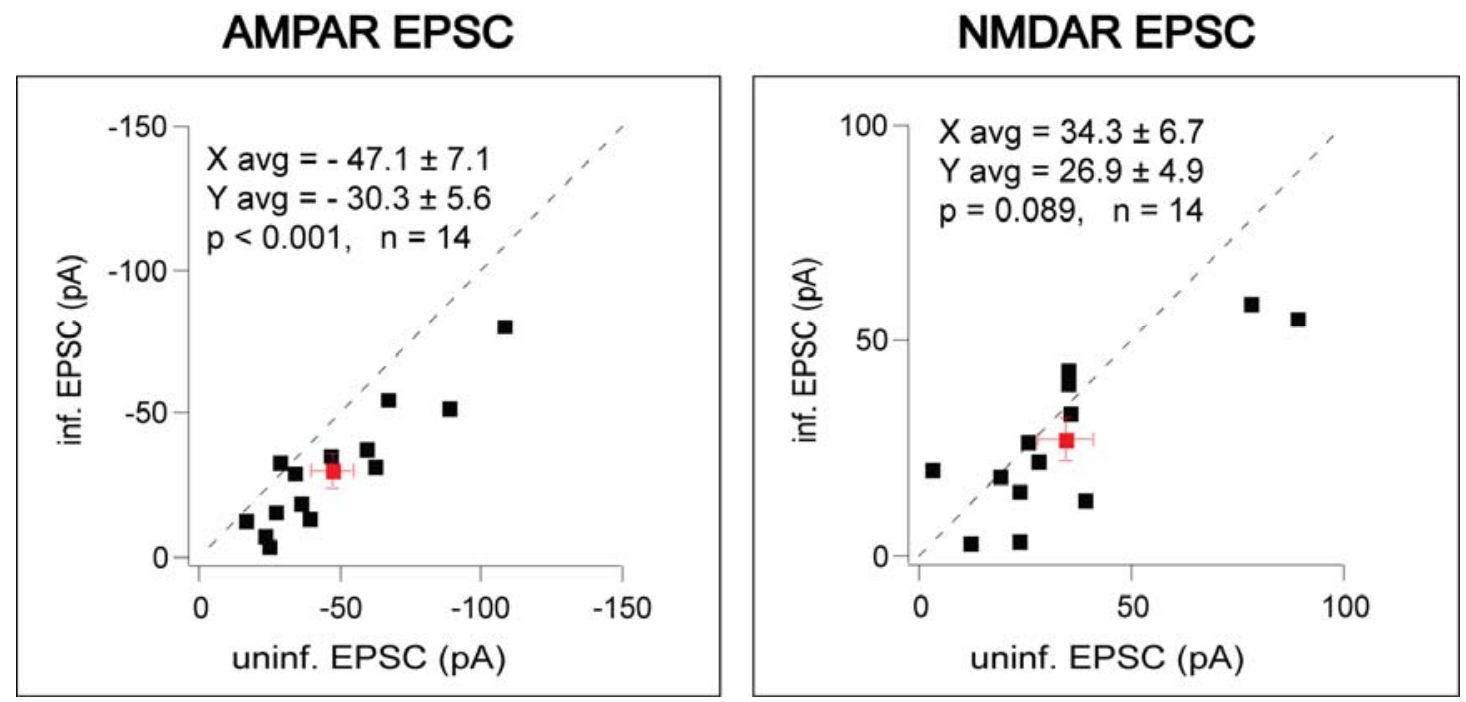

Figure 13. Effects of PSD-95 PDZ12 molecular replacement on basal synaptic transmission.

A) Illustration depicting the PSD-95 PDZ12 molecular replacement vector. B) Dual-whole cell evoked EPSCs recorded from an untransfected control neuron and a PSD-95 knockdown simultaneously replaced with overexpressing wild-type PSD-95 PDZ12 neighbor. Distributions show a significant decrease in AMPAR-EPSC amplitudes (left) with no significant change in NMDAR-EPSC amplitudes (right).

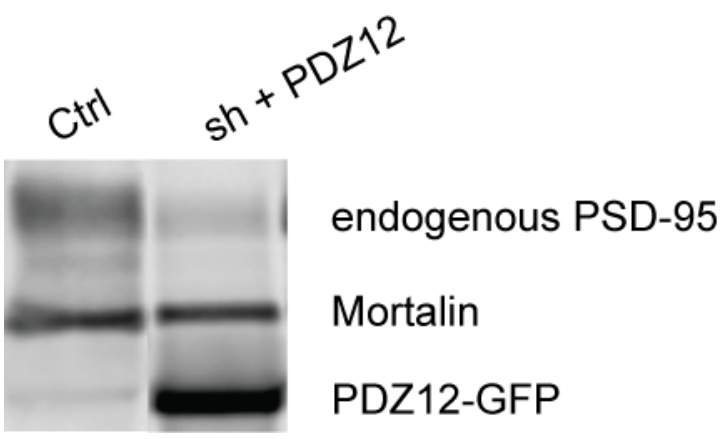

Figure 14. Molecular replacement by PSD-95 PDZ12 in rat hippocampal dissociated cultures assessed by western-blot.

A decrease in the endogenous level of PSD-95 and a robust expression of GFP-fused PSD-95 PDZ12 can be observed. 


\section{III.A.5. Molecular replacement by PSD-95 lacking its PDZ3 domain}

It is already known that the molecular replacement by PSD-95 expressing its N-terminal part in combination with its two first PDZ domains (PDZ12) or in conjugation with its three PDZ domains ( $\triangle \mathrm{SH} 3-\mathrm{GK})$ is not able to rescue the knockdown effect mediated by shRNA against endogenous PSD-95 and consequently leads to a 35 to $45 \%$ decrease in AMPAR-transmission strength (Schlüter et al., 2006). This implies that PDZ3 domain by itself is not required for PSD-95 to be functional. To show the non-requirement of the PDZ3 domain for rescuing basal synaptic transmission, the most straightforward approach was to record electrophysiological properties of neurons expressing PSD-95 missing its PDZ3 domain in a PSD-95 knocked-down background. The results showed a 2.2fold increase in AMPAR-EPSC amplitude with no change in NMDAR-EPSC (Figure 15). Western blot data showed an efficient knockdown of endogenous PSD-95 concomitant to a high expression level of PSD-95 PDZ3 (Figure 16).This indicates that the molecular replacement used in this experiment is operational, and thus supports the above-mentioned electrophysiological results.

Altogether, this illustrates that the PDZ3 domain of PSD-95 is not a requisite for the protein to regulate basal synaptic transmission in CA1 region hippocampal pyramidal cells. 
A.

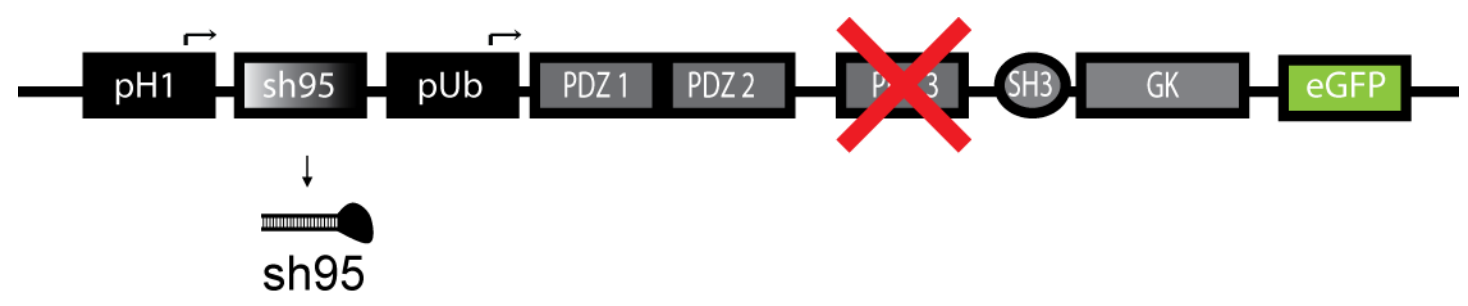

B.

AMPAR EPSC

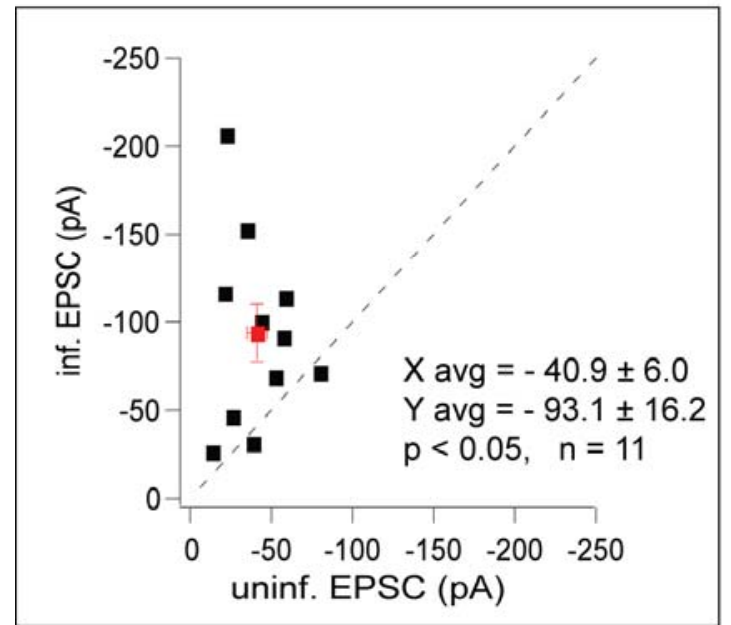

NMDAR EPSC

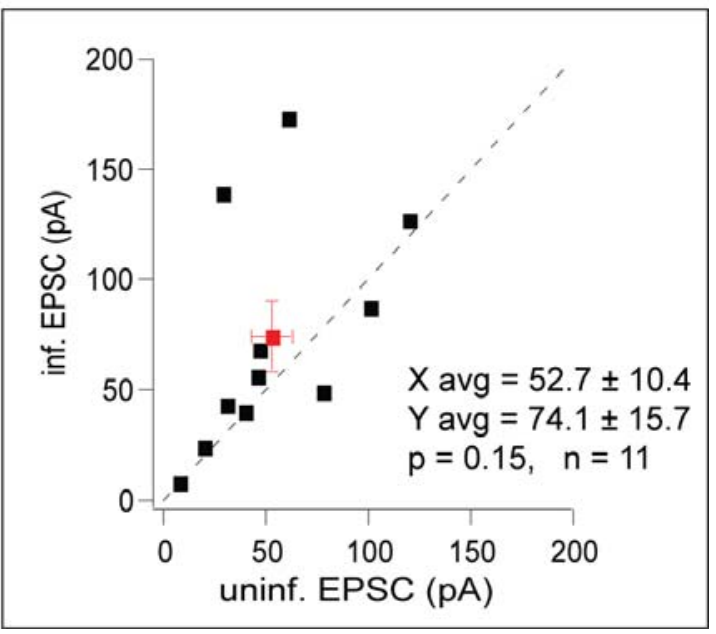

Figure 15. Effects of PSD-95 PDZ3 molecular replacement on basal synaptic transmission.

A) Illustration depicting the PSD-95 2 PDZ3molecular replacement vector. B) Dual-whole cell evoked EPSCs recorded from an untransfected control neuron and a PSD-95 knockdown simultaneously replaced with overexpressing PSD-95 PDZ3 neighbor. Distributions show a significant increase in AMPAR-EPSC amplitudes (left) with no significant change in NMDAR-EPSC amplitudes (right).

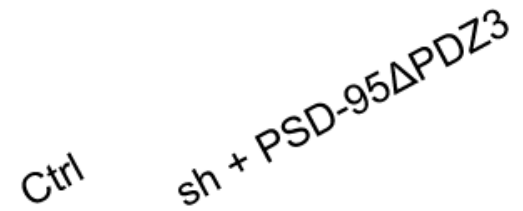

\section{PSD-95 $\triangle$ PDZ3-GFP}

endogenous PSD-95

Mortalin 
Figure 16. Molecular replacement by PSD-95 PDZ3 in rat hippocampal dissociated cultures assessed by western-blot.

A decrease in the endogenous level of PSD-95 and a robust expression of GFP-fused PSD$95 \Delta$ PDZ3 can be observed.

\section{III.A.6. Molecular replacement by PSD-95 lacking its SH3 domain}

The next step was to determine to what extent PSD-95 relies on its Cterminal motif to localize AMPARs at synapses. In order to examine this, I expressed a mutant PSD-95 construct lacking its SH3 domain and recorded its electrophyiological properties on a PSD-95 knockdown background. As a result, I observed an enhancement of AMPAR synaptic strength by 1.9-fold, while NMDAR transmission was increased by 1.3 -fold. (Figure 17) These results are similar to the ones obtained from molecular replacement with full-length PSD95, thus suggesting this is a fully functional PSD-95. Western blot data showed an efficient knockdown of endogenous PSD-95 concomitant to a high expression level of PSD-95 $\Delta$ SH3 (Figure 18). This indicates that the molecular replacement used in this experiment is operational, and thus supports the above mentioned electrophysiological results.

Altogether, this data demonstrates that the SH3 domain of PSD-95 is not necessary for influencing basal synaptic transmission. 
A.

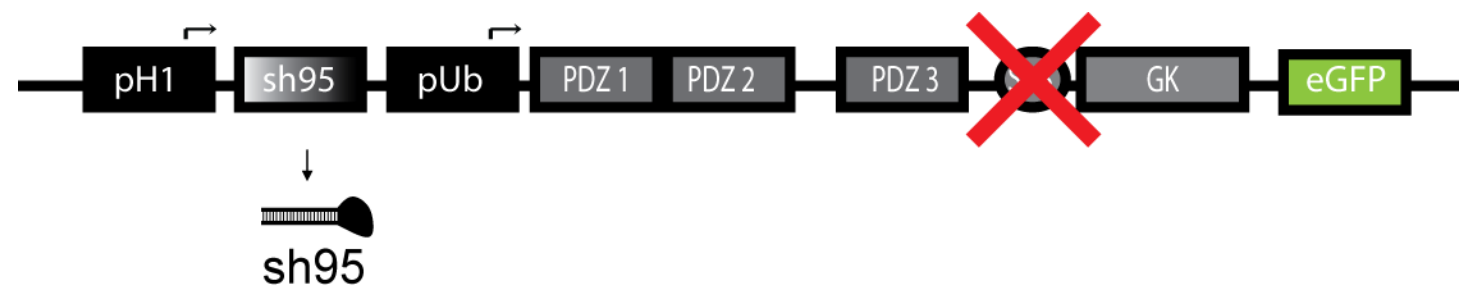

B.

AMPAR EPSC

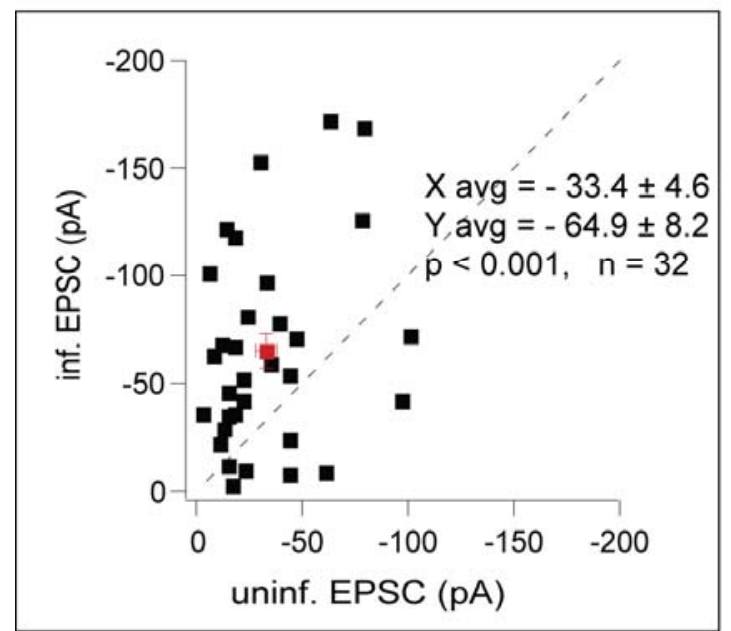

NMDAR EPSC

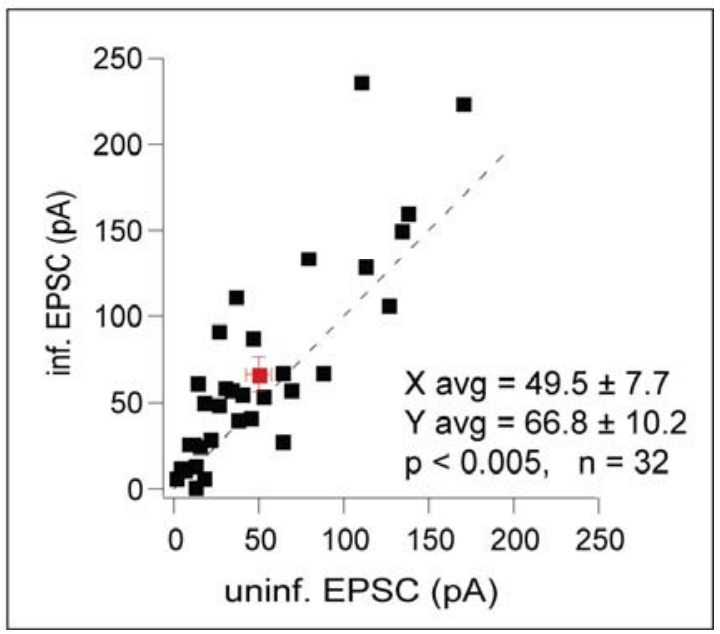

Figure 17. Effects of PSD-95 $\Delta \mathrm{SH} 3$ molecular replacement in basal synaptic transmission.

A) Illustration depicting the PSD-95 $\mathrm{SH} 3$ molecular replacement vector by. B) Dual-whole cell evoked EPSCs recorded from an untransfected control neuron and a PSD-95 knockdown simultaneously replaced with PSD-95ASH3 overexpressing neighbor. Distributions show a significant increase in AMPAR-EPSC amplitudes (left) concomitant with an increase in NMDAREPSC amplitudes (right). 


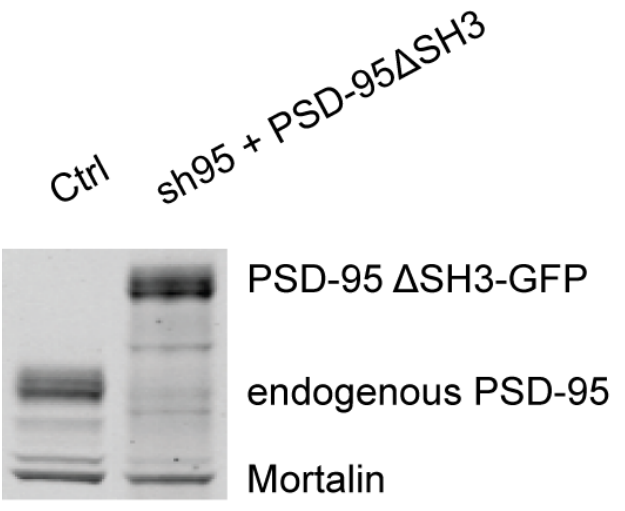

Figure 18. Molecular replacement by PSD-95 $\Delta$ SH3 in rat hippocampal dissociated cultures assessed by western-blot.

A decrease in the endogenous level of PSD-95 and a robust expression of GFP-fused PSD-95 $\Delta$ SH3 can be observed.

\section{III.A.7. Molecular replacement by PSD-95 lacking its GK domain}

To test the involvement of the GK domain in order to determine the role of the C-terminal region more narrowly, I next selectively deleted the GK domain from PSD-95. This led to a partial rescue of AMPAR and NMDAR synaptic strength where each component was brought to the control cell level (Figure 19). From the western-blot, one can observe that the expression level of this construct is not as high and that the endogenous PSD-95 is not as clearly knocked-down (Figure 20). Direct comparison among the different constructs shows that PSD-95 $\Delta$ GK expression level is not as strong as the PSD-95 SH3 or full-length PSD-95 constructs (Figure 21). Moreover, the endogenous PSD-95 is not as strongly knocked-down compared to the other previous constructs (Figure 21). 
A.

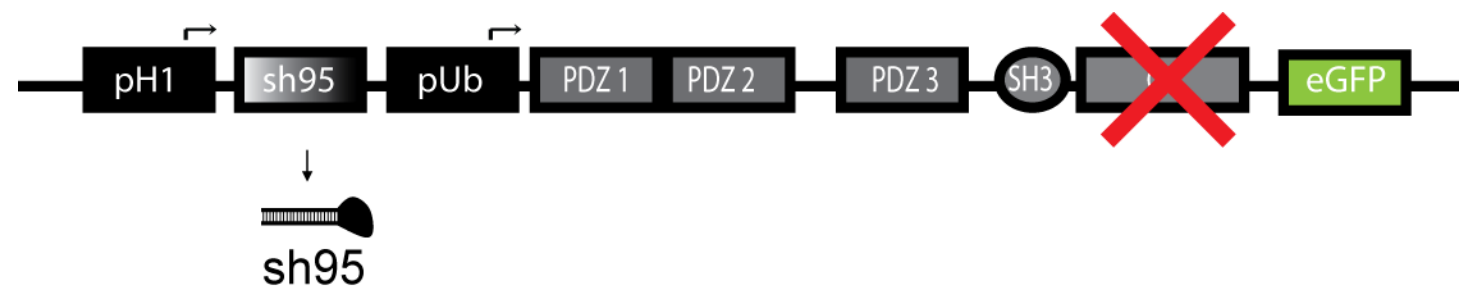

B.

AMPAR EPSC

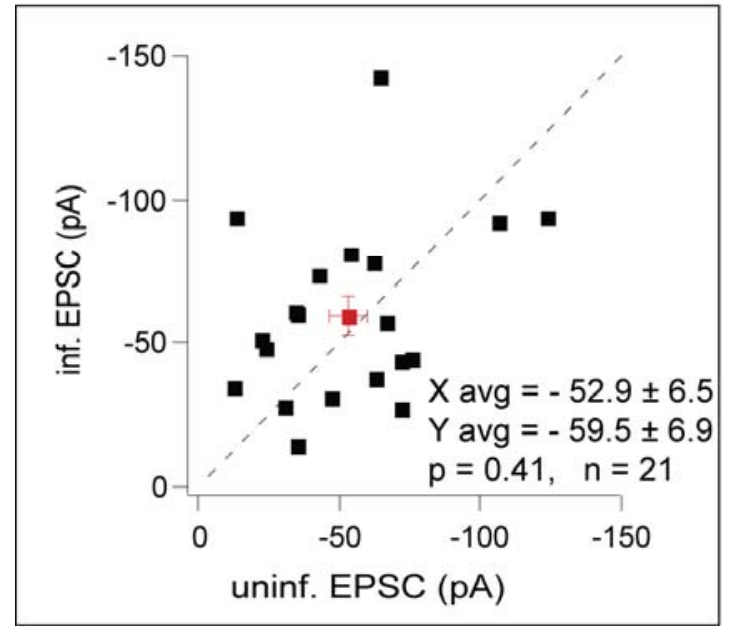

NMDAR EPSC

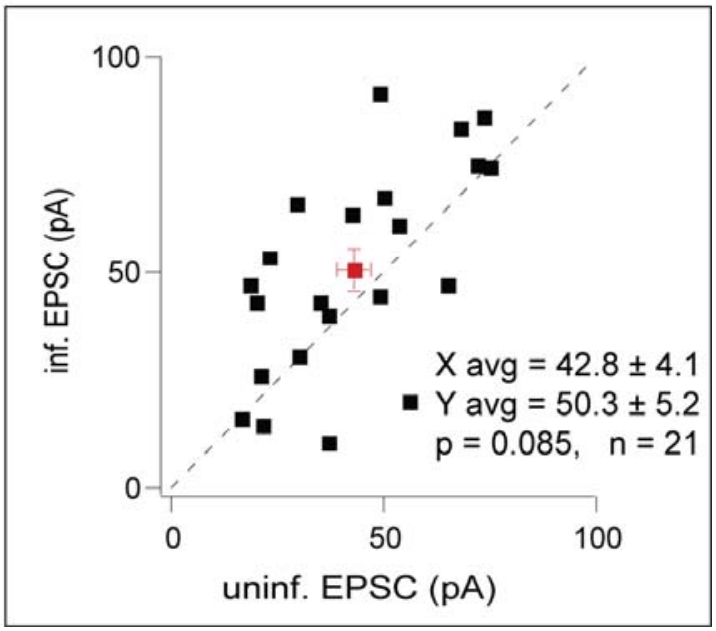

Figure 19. Effects of PSD-95 $\Delta$ GK molecular replacement on basal synaptic transmission.

A) Illustration depicting the PSD-95 GGK molecular replacement vector. B) Dual-whole cell evoked EPSCs recorded from an untransfected control neuron and a PSD-95 knockdown simultaneously replaced with PSD-95 $\Delta$ GK overexpressing neighbor. Distributions show no 


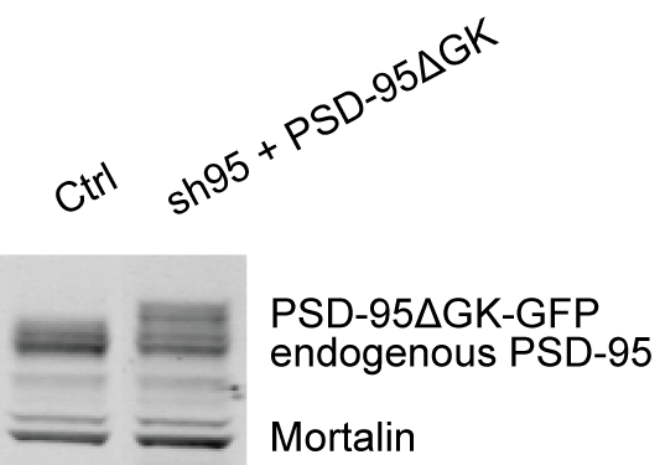

Figure 20. Molecular replacement by PSD-95 $\Delta$ GK in rat hippocampal dissociated cultures assessed by western-blot.

The endogenous level of PSD-95 is not as strongly decreased, and the PSD-95 $\Delta$ GK is not as strongly expressed.

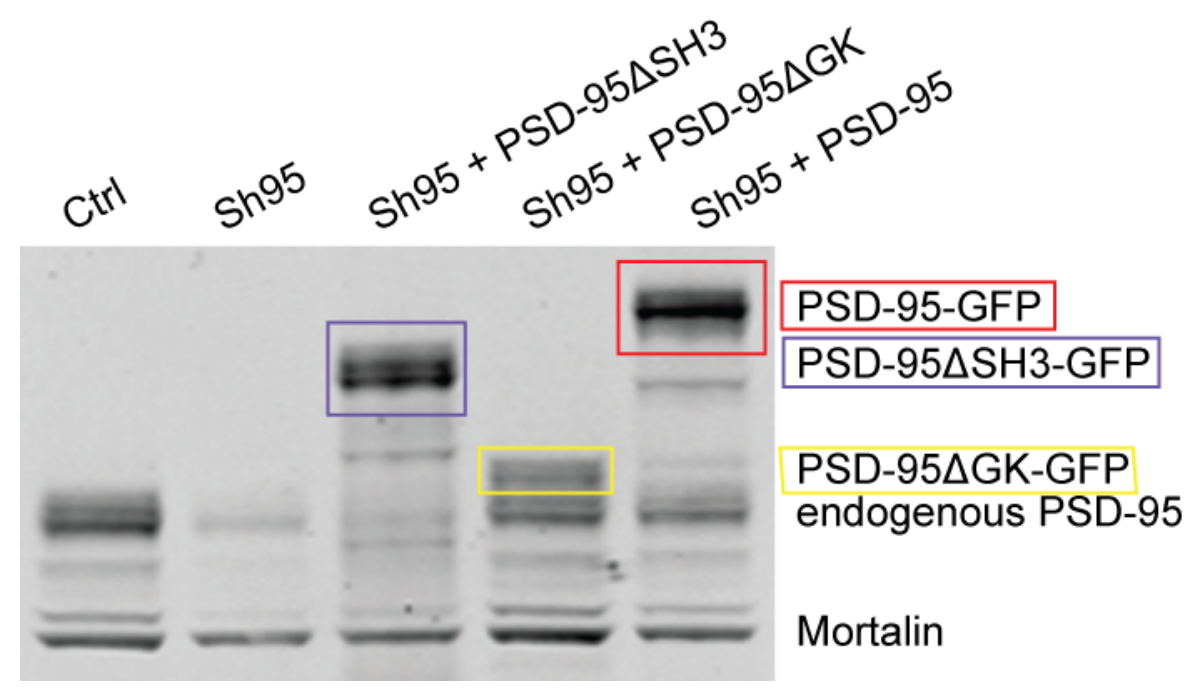

Figure 21.Comparison of the different knockdown effects and expression levels of each truncated form of PSD-95 molecular replacement vector.

The knockdown effect mediated by the PSD-95 $\mathrm{GK}$ molecular replacement vector is not as strong as the PSD-95 $\Delta$ SH3 molecular replacement vector. The expression level of PSD-95 $\Delta \mathrm{GK}$ is also lower than PSD-95 SSH3 or PSD-95.

Altogether, these results support the participation of the GK domain of PSD-95 in influencing basal synaptic transmission. 
This intermediate result motivated the next three experiments.

- Firstly, based on the electrophysiological data of the molecular replacement by PSD-95 $\Delta \mathrm{GK}$ (partial rescue in basal synaptic transmission), I tried another approach. Briefly, the idea was to test the role of PSD-95 by expressing PDZ12-GK in basal synaptic transmission, in the molecular replacement context. The rational behind this experiment is explained in paragraph III.A.8).

- Secondly, based on the western-blot data showing that the PSD-95 $\Delta$ GK protein is not so strongly expressed, I questioned the functionality of the recombinant and tested this possibility by overexpressing PSD-95 $\Delta$ GK in a wild-type neuron (explanations more detailed in the paragraph III.A.9)

- Lastly, based on the western blot data showing a definite, but not as strong, knockdown effect of the endogenous PSD-95, I questioned the role played by the remaining endogenous PSD-95. I tested this hypothesis by taking advantage of the PSD-95 KO mouse (see paragraph III.B). 


\section{III.A.8. Molecular replacement using a mutant form of PSD-95 expressing PDZ12 and GK domains}

Since the replacement of endogenous PSD-95 by its mutant lacking the GK domain gave a partial rescue (Figure 20), the interpretation of the results is more difficult in terms of concluding whether the GK domain is crucial for basal synaptic transmission or not. In order to better assess this question, I tried another approach.

I already showed that PSD-95 PDZ12 is not functional in the absence of PSD-95 (50\% decrease in AMPAR-evoked current) (Figure 14). So, what if GK domain is appended to this part of the protein? Could it then rescue the AMPAR function?

With this aim, I analyzed the properties of a mutant PSD-95 expressing the N-terminal and the two first PDZ domains, followed by the GK domain 
(PDZ12-GK). The PDZ12-GK was able to rescue AMPAR transmission to the control cells level, similar to PSD-95 $\Delta \mathrm{GK}$, but not more (Figure 22). The western blot result shows that the PDZ12-GK construct had a strong expression level, however the knockdown effect could not be evaluated as this truncated form of PSD-95 runs at the same size as the endogenous one (Figure 23). This suggests that the observed effects at the electrophysiological level are related to the function of the truncated protein rather than to its poor expression levels.

Altogether, these data show that appending the GK domain to a nonfunctional PSD-95 (PDZ12) resulted in a partial rescue of basal synaptic transmission when endogenous PSD-95 is reduced. This supports a potential role for GK domain with respect to synaptic strength regulation mediated by PSD-95.

A.

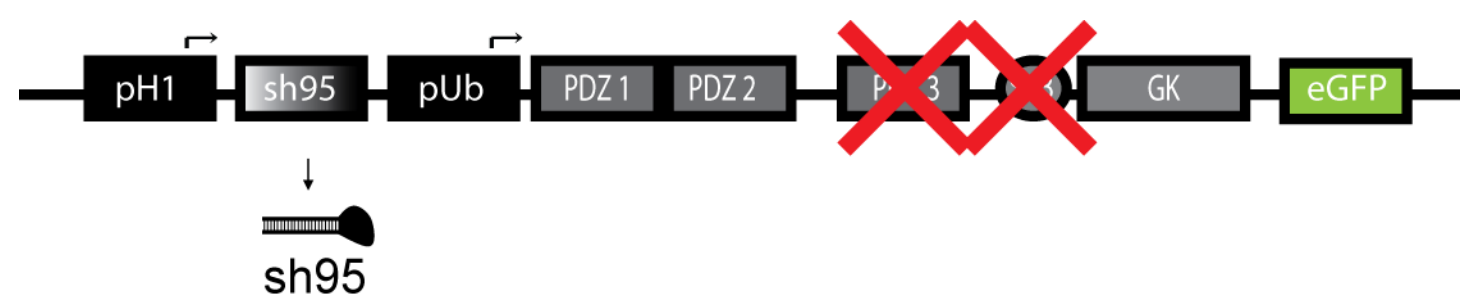

B. 

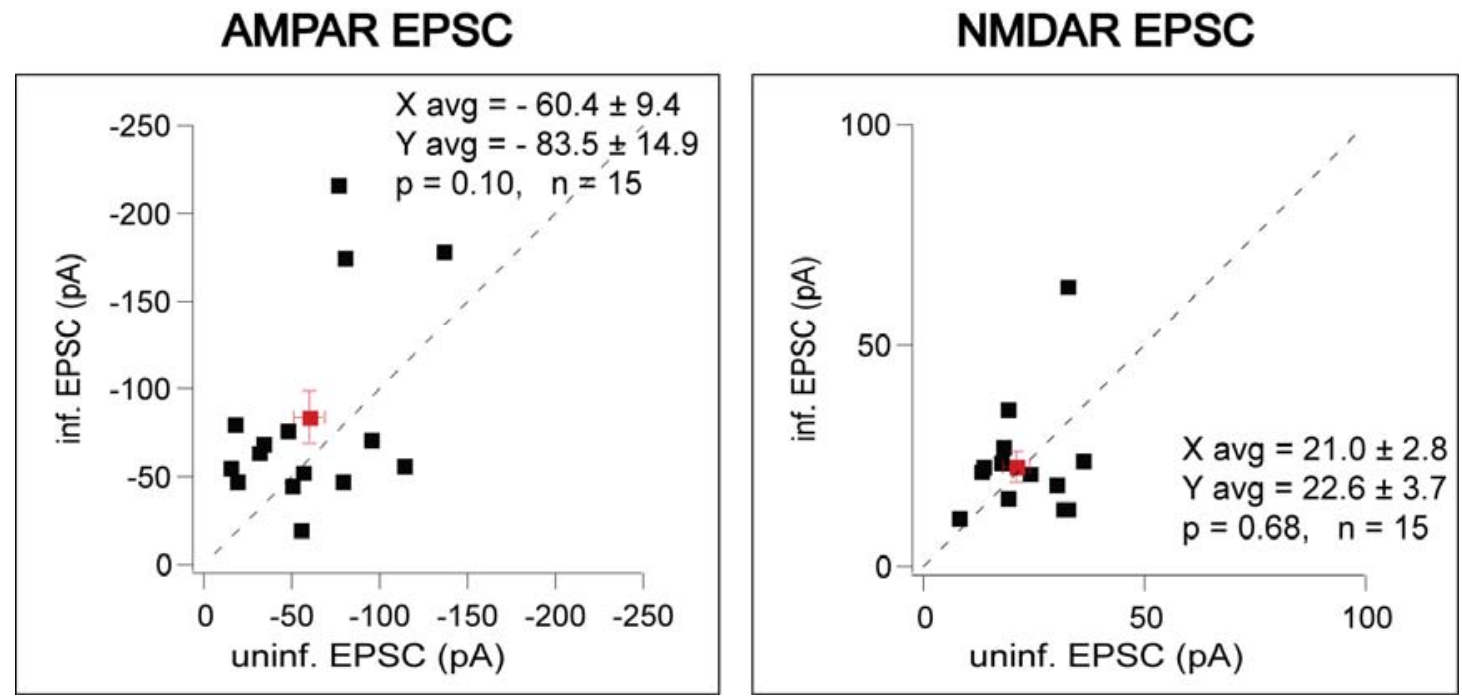

Figure 22. Effects of PSD-95 PDZ12-GK molecular replacement on basal synaptic transmission.

A) Illustration depicting the PSD-95 PDZ12-GK molecular replacement vector . B) Dual-whole cell evoked EPSCs recorded from an untransfected control neuron and a PSD-95 knockdown replaced with PSD-95 PDZ12-GK overexpressing neighbor. Distributions show no significant change in AMPAR-EPSC amplitudes (left) or in NMDAR-EPSC amplitudes (right).

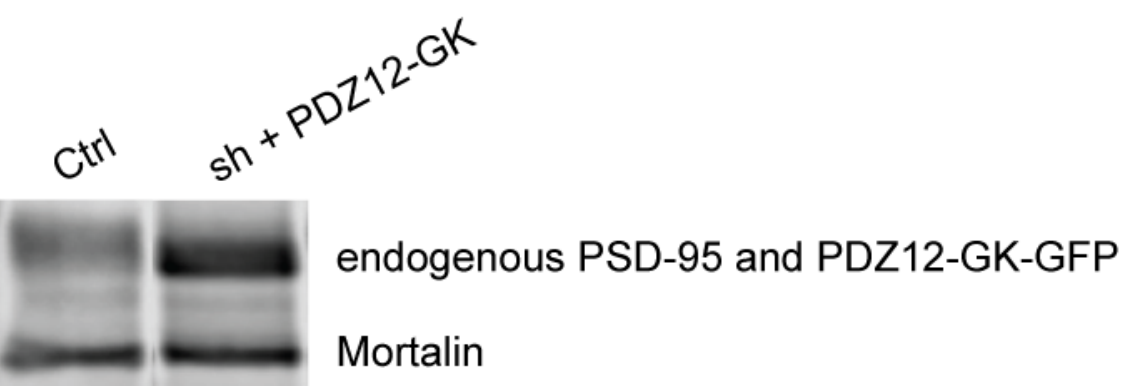

Figure 23. Molecular replacement by PSD-95 PDZ12-GK in rat hippocampal dissociated cultures assessed by western-blot.

The endogenous level of PSD-95 cannot be assessed as the mutant protein runs at the same size as the endogenous PSD-95. Though, a robust expression of GFP-fused PSD-95 PDZ12-GK can be observed.

\section{III.A.9. Overexpression of PSD-95 lacking its GK domain or containing only PDZ12-GK domains}


Although, the expression level doesn't seem to be the limiting parameter for the construct to exert its effect on basal synaptic transmission, one can question the appropriate folding and stability of the recombinant proteins., for example for the PSD-95 lacking its GK domain or only containing PDZ12-GK domains.

The functionality and stability of a mutant can be tested by overexpressing it in a wild type neuron and observing its ability to regulate AMPAR- and NMDARmediated transmission.

Overexpressing PSD-95 GKK or PDZ12-GK show that AMPAR-evoked currents were increased by 2.4-fold and 2-fold, respectively, with no significant alteration in the NMDAR component in both cases (Figure 24, Figure 26). These results are similar to the one obtained with molecular replacement by full-length PSD-95. In addition, western blot results show a robust overexpression of the respective constructs (Figure 25, Figure 27), corroborating the electrophysiological data. Altogether, these results demonstrate the functionality of the PSD-95 $\Delta$ GK and PDZ12-GK mutants, making it unlikely that the molecular replacement of these constructs rescued AMPAR-transmission to the control cells level (with no further enhancement) uniquely because the mutant was only partially functional or stable.

A. 


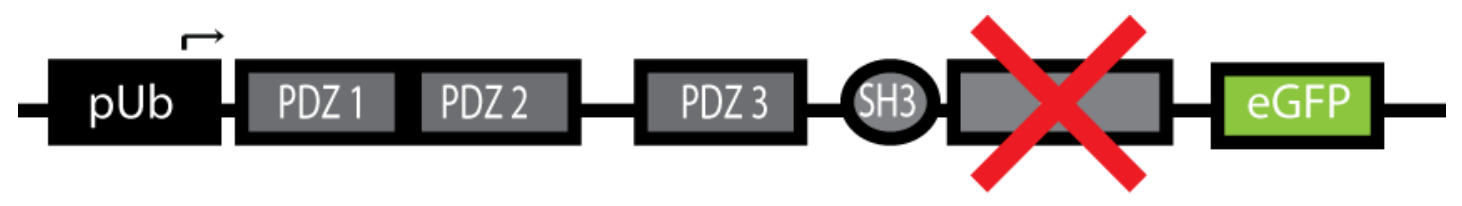

B.

AMPAR EPSC

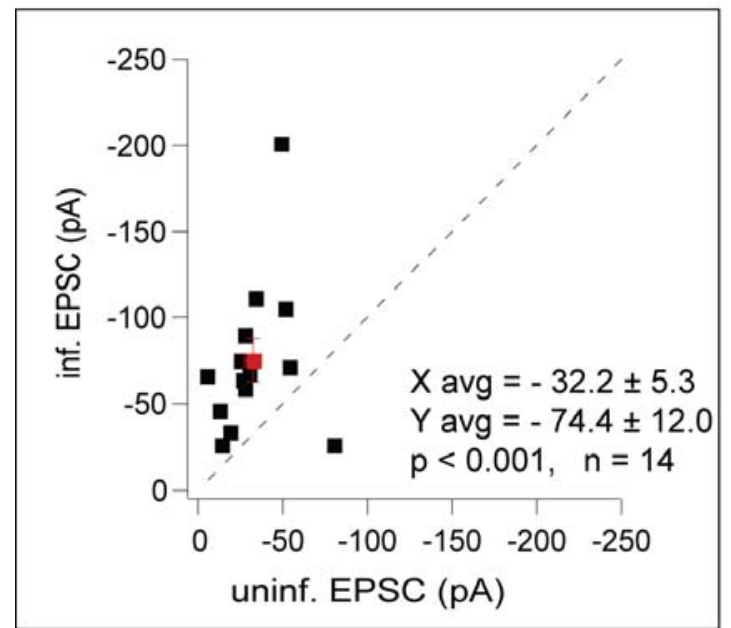

NMDAR EPSC

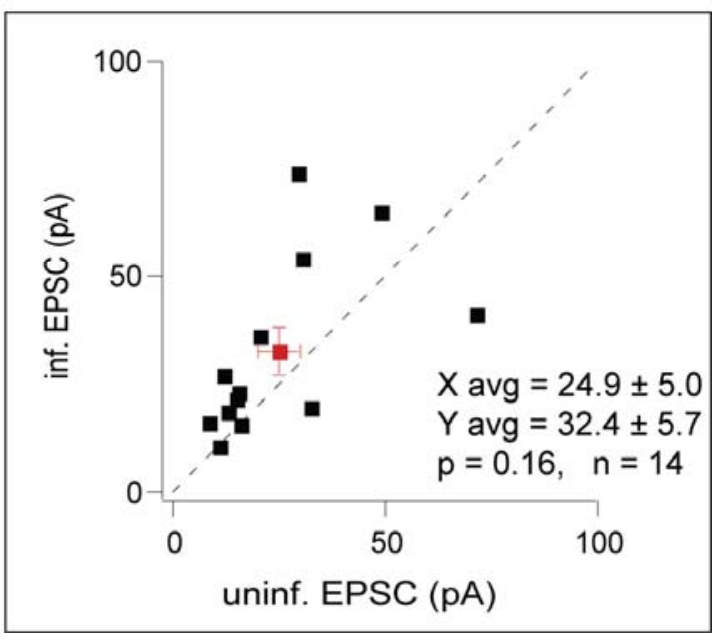

Figure 24. Effects of PSD-95 $\Delta$ GK overexpression on basal synaptic transmission.

A) Illustration depicting the PSD-95 $\Delta$ GK overexpression vector. B) Dual-whole cell evoked EPSCs recorded from an untransfected control neuron and a PSD-95 knockdown replaced with PSD95 $\triangle \mathrm{GK}$ overexpressing neighbor. Distributions show an increase in AMPAR-EPSC amplitudes (left) and no significant change in NMDAR-EPSC amplitudes (right).

\section{Ctri OED $D^{G^{K}}$}

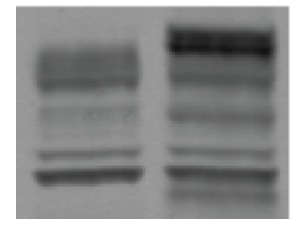

PSD-95 $\triangle$ GK-GFP

endogenous PSD-95

Mortalin

Figure 25. Overexpression of PSD-95 $\Delta \mathrm{GK}$ in rat hippocampal dissociated cultures assessed by western-blot.

The endogenous level of PSD-95 is similar to the one observed in control cell. In addition, a robust expression of GFP-fused PSD-95 $\Delta$ GK can be observed. 
AMPAR EPSC

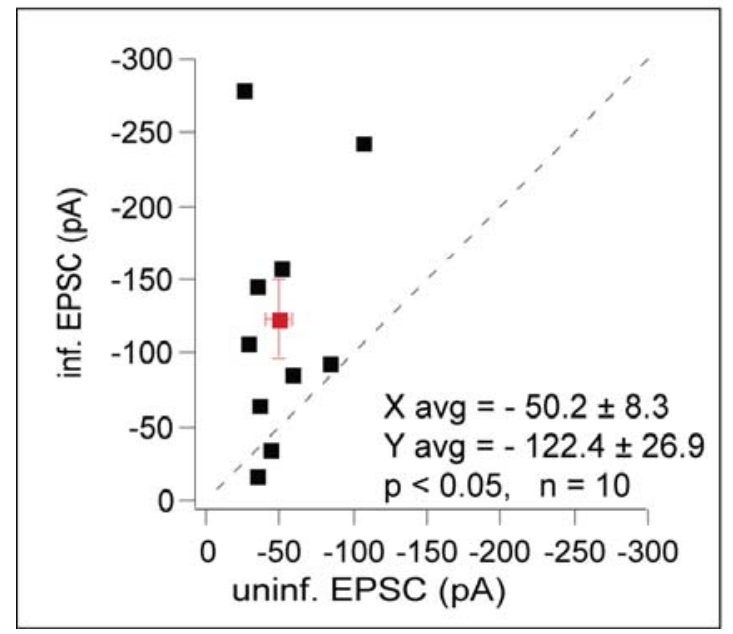

NMDAR EPSC

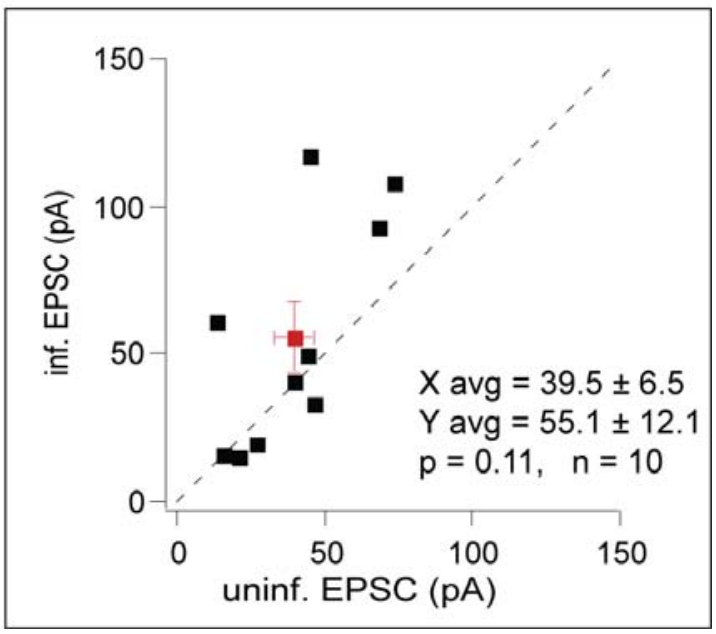

Figure 26. Effects of PSD-95 PDZ12-GK overexpression on basal synaptic transmission.

A) Illustration depicting the PSD-95 PDZ12-GK overexpression vector. B) Dual-whole cell evoked EPSCs recorded from an untransfected control neuron and a PSD-95 knockdown replaced with PSD-95 PDZ12-GK overexpressing neighbor. Distributions show an increase in AMPAR-EPSC amplitudes (left) and no significant change in NMDAR-EPSC amplitudes (right).

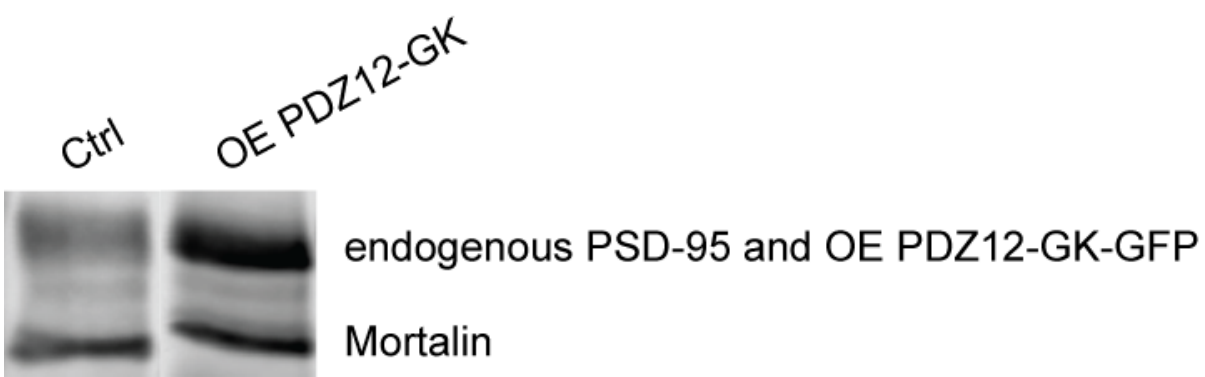

Figure 27. Overexpression of PSD-95 PDZ12 in rat hippocampal dissociated cultures assessed by western-blot.

The endogenous level of PSD-95 cannot be assessed as the mutant protein runs at the same size as the endogenous PSD-95. In addition, a robust expression of GFP-fused PSD-95 PDZ12-GK can be observed. 
From this point on, I reiterated the molecular replacement with the recombinant PSD-95 lacking its GK domain. Very surprisingly, some experiments showed a significant enhancement in AMPAR-EPSC (Figure 28 panel B), which is comparable to the full-length and functional PSD-95. To my surprise, when the experiment was again performed, it showed a partial rescue of AMPAR transmission to control cell level (Figure 28 panel C) as previously seen in the first batch of recordings (Figure 19). However, when pulled altogether, one can observe a significant 1.4 fold increase in AMPAR-evoked currents and 1.3-fold enhancement in NMDAR-EPSCs (Figure 28 panel D). This variability in the results was correlated with separate batches of animals. Indeed, each set of recordings was performed by using a different animal. Although, the animals were used at the age of P8 for each experiment, one cannot exclude a slightly faster or slower maturation process related to each animal condition that could influence the outcome of the results. 
A.

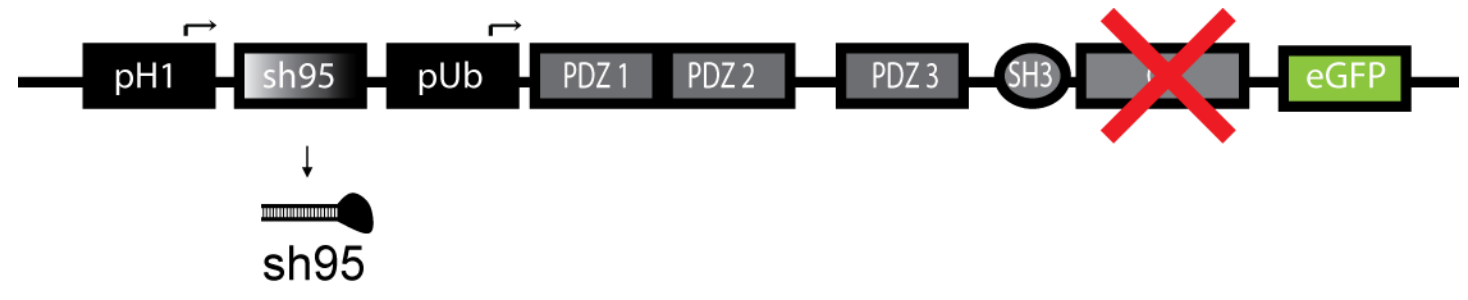

B.

AMPAR EPSC

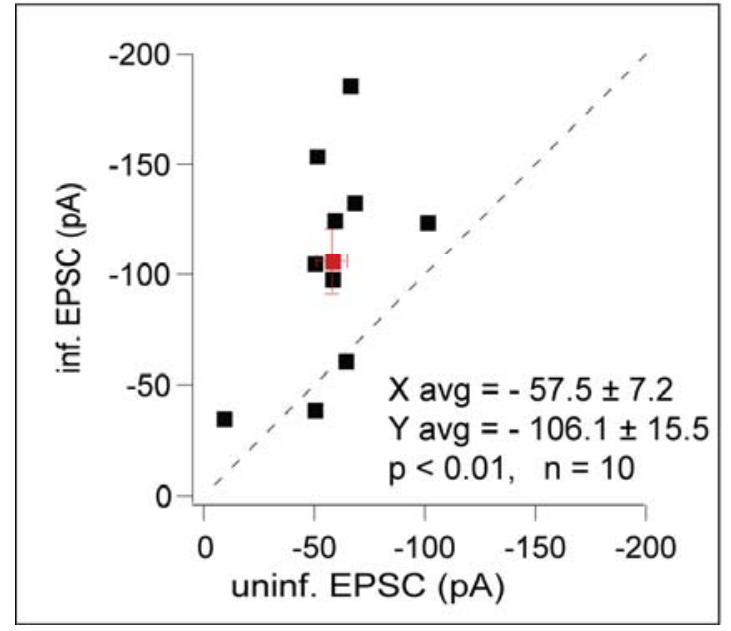

C

AMPAR EPSC

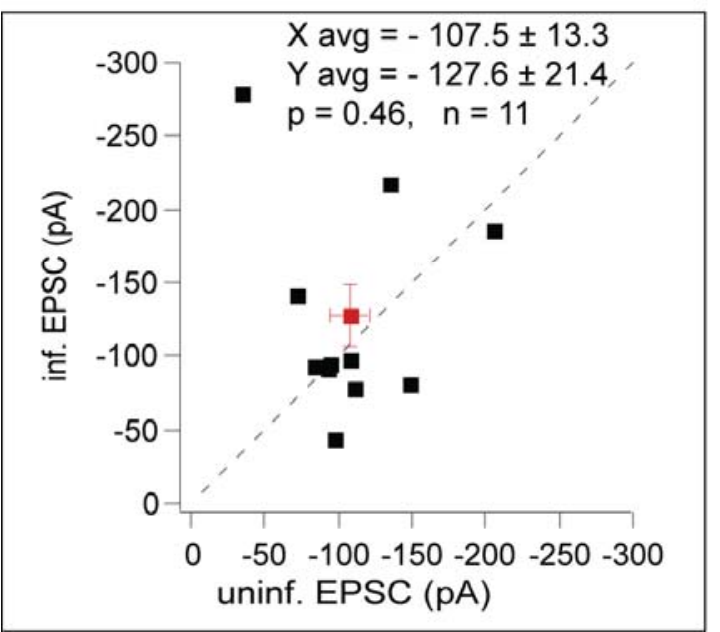

NMDAR EPSC

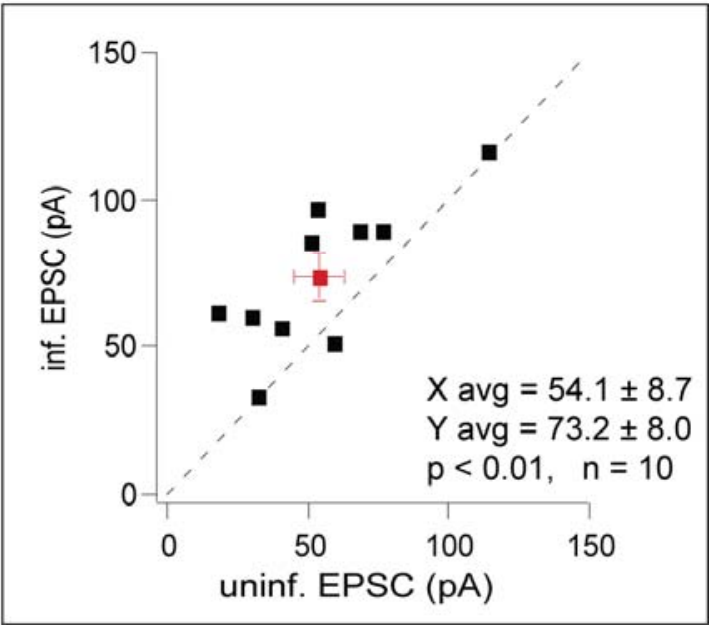

NMDAR EPSC

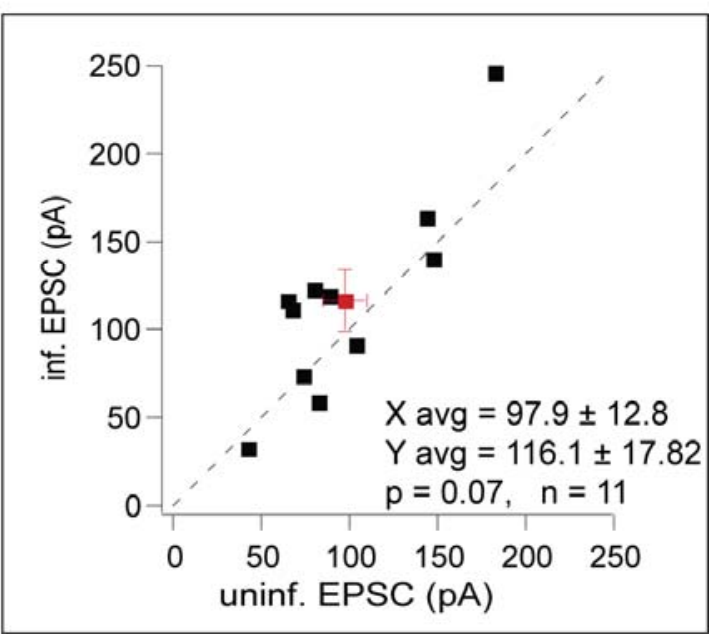

D 
AMPAR EPSC

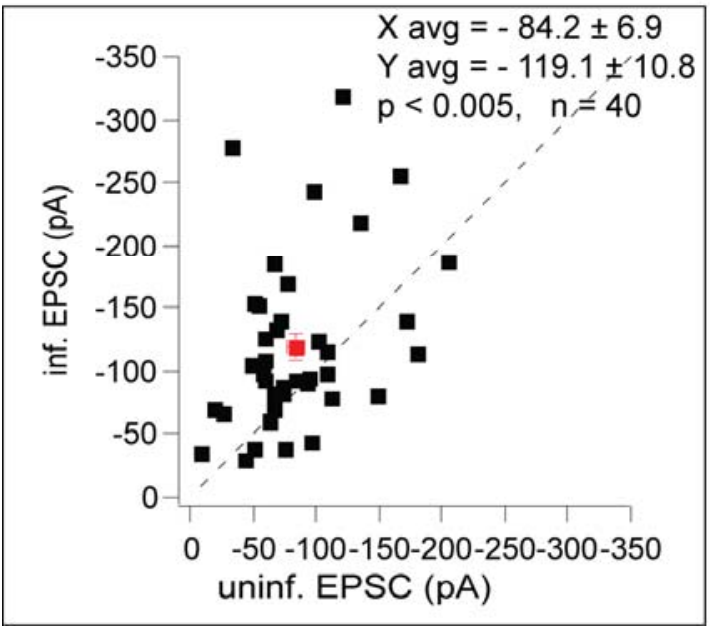

NMDAR EPSC

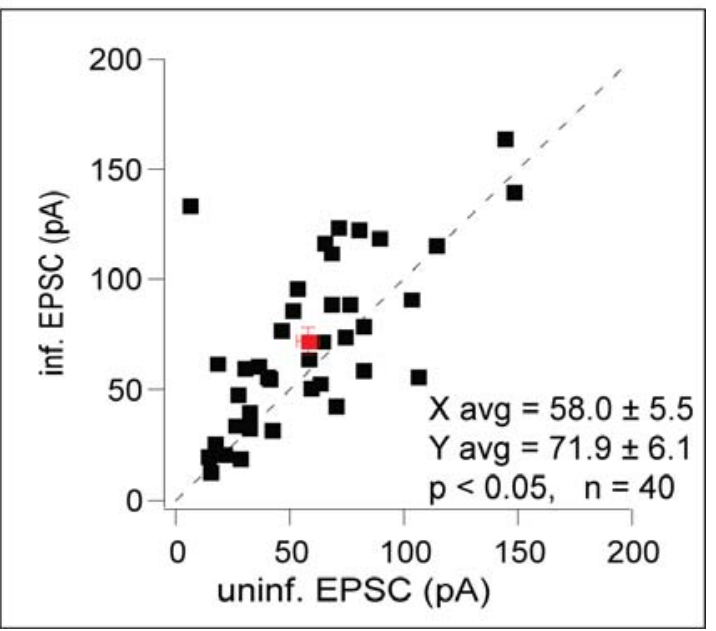

Figure 28. Variability of the results observed with PSD-95 $\Delta$ GK molecular replacement.

A) Illustration depicting molecular replacement by PSD-95 GGK. B) Dual-whole cell evoked EPSCs recorded from an untransfected control neuron and a PSD-95 knockdown simultaneously replaced with PSD-95 PDZ12-GK overexpressing neighbor, in one batch of recordings. Distributions show an increase in AMPAR-EPSC amplitudes (left) and NMDAR-EPSC amplitudes (right). C) Molecular replacement by PSD-95 $\triangle \mathrm{GK}$, in another batch of recordings. AMPAR- and NMDAR-transmission are unchanged while replacing endogenous PSD-95 by its mutant form lacking GK domain. D) Combined experiments of molecular replacement by PSD-95 $\Delta$ GK expressing neurons. A 1.4 fold increase is observed AMPAR-evoked currents accompanied with a 1.3 fold increase in NMDAR-EPSCs. 


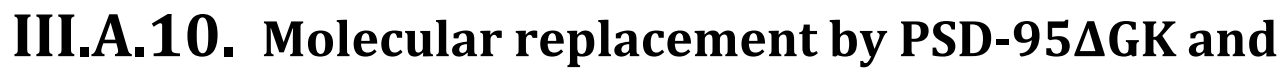 activity manipulation}

In order to test whether the variability of the results is caused by differences in the activity of the slices, dual whole cell recordings using molecular replacement by PSD-95 $\Delta$ GK were performed as before, but with a variation concerning the feeding media of the slice. In this experiment, directly after injection of the lentivirus, the slice culture medium was supplemented with Bicucculline $(20 \mu \mathrm{M})$ or APV $(25 \mu \mathrm{M})$. Bicucculine is a GABAa channel antagonist, and thus was used to increase the spontaneous excitatory synaptic activity in the slice cultures. On the other hand, APV is a NMDAR antagonist, and was used to reduce the overall excitatory activity of the slice. None of these pharmalogical activity manipulations led to a significant change when compared to non-treated slices. Control and infected cells had similar responses overall to these activity manipulations in the slices (Figure 29). This suggests that the observed variability in the PSD-95 $\Delta$ GK results were likely not linked to different activity levels within the different slice cultures.

A.

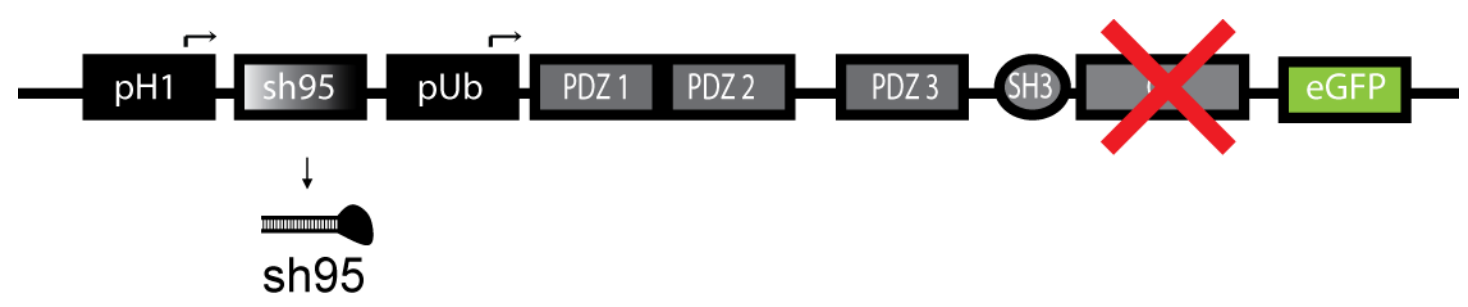


B.

AMPAR EPSC

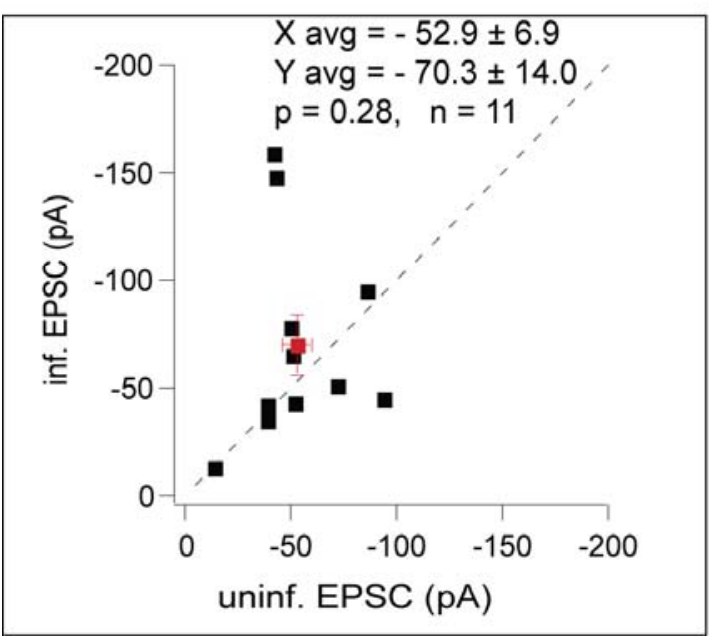

C.

\section{AMPAR EPSC}

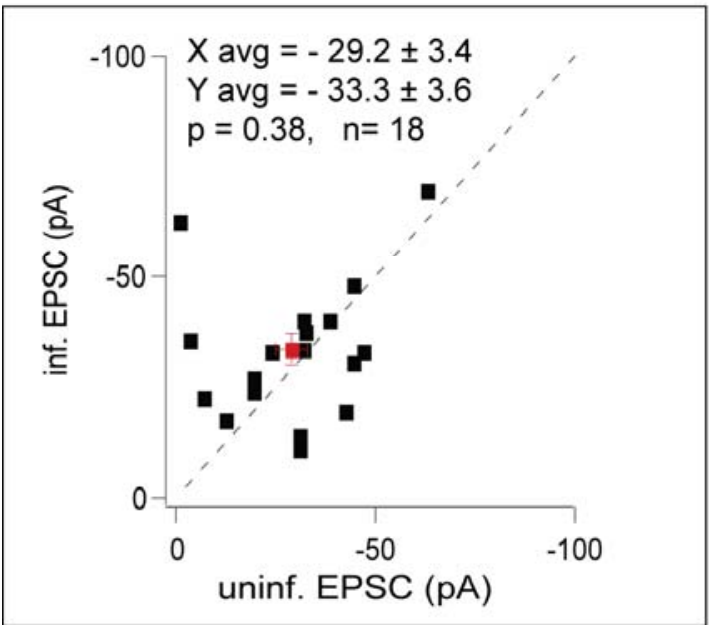

NMDAR EPSC

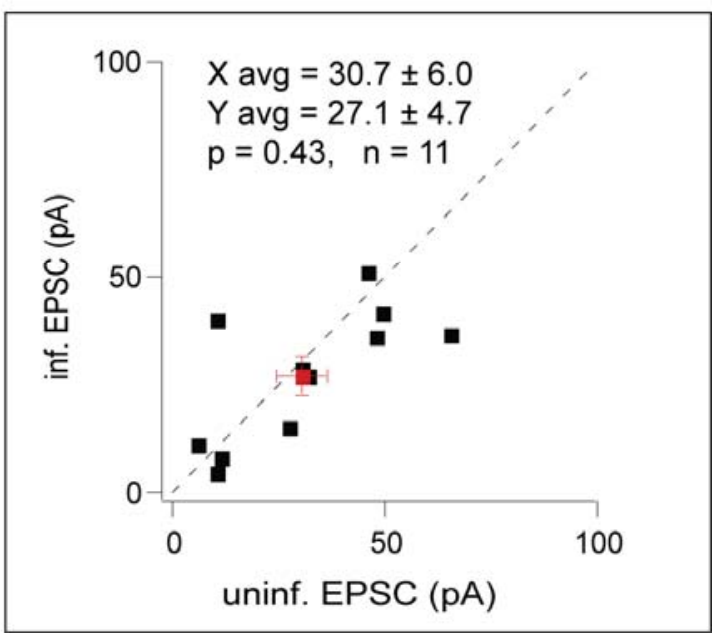

Figure 29. Effects of activity manipulations on PSD-95 GGK molecular replacement on basal synatptic transmission.

A) Illustration depicting the PSD-95 $\Delta \mathrm{GK}$ molecular replacement vector. B) Effects of activity manipulation (Bicucculine - $20 \mu \mathrm{M}$ ) onto PSD-95 $\Delta \mathrm{GK}$ molecular replacement on basal synaptic transmission. Distributions show no change in AMPAR-EPSC amplitudes (left) or in NMDAR-EPSC amplitudes (right). C) Effects of activity manipulation (APV - 25 $\mu$ M) onto PSD-95 $\Delta$ GK molecular replacement on basal synaptic transmission. Distributions show no change in AMPAR-EPSC 
amplitudes (left) or in NMDAR-EPSC amplitudes (right).

According to this set of experiments, a puzzling question remained. Why did constructs including C-terminal domains, but not constructs with only the PDZ domains, rescue AMPAR function to control cell levels.

One concern was the efficiency of the shRNA against PSD-95. As it is already known that PSD-95 has the ability to multimerize with itself at its very $\mathrm{N}$ terminal site in a head to head manner, one can speculate that if endogenous PSD-95 is not properly knocked-down, the protein will still have the opportunity to multimerize between the truncated protein (PSD-95 $\Delta$ GK for example) and the full length PSD-95. Due to this binding, the shortened version would still be brought to the synapse, and the combination of both types may still influence basal synaptic transmission.

To definitively rule out any contribution of endogenous PSD-95, I decided to take advantage of the PSD-95 knockout mouse line we possess (Yao et al., 2004). Therefore, I repeated the following electrophysiological recordings using the knockout mouse organotypic slice model.

\section{III.B. Overexpression studies in PSD-95 KO mouse hippocampal organotypic slice cultures}

\section{III.B.1. Expression of PSD-95 PDZ12 in PSD-95 KO neurons}

First, it was important to confirm that PSD-95 PDZ12 was not able to rescue basal synaptic transmission in the PSD-95 KO mouse, similar to when 
PSD-95 is knocked-down by shRNA technology in the rat model (Figure 13). The results show that expression of PDZ12 alone neither changed AMPAR function nor NMDAR function in comparison to control PSD-95 KO neurons (Figure 30). These results indicate that PSD-95 PDZ12 alone is not functional, similar to what was observed in the rat system.

A.

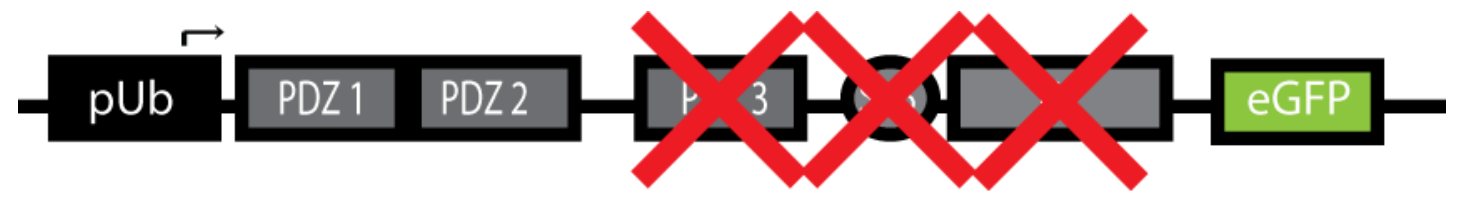

B.

AMPAR EPSC

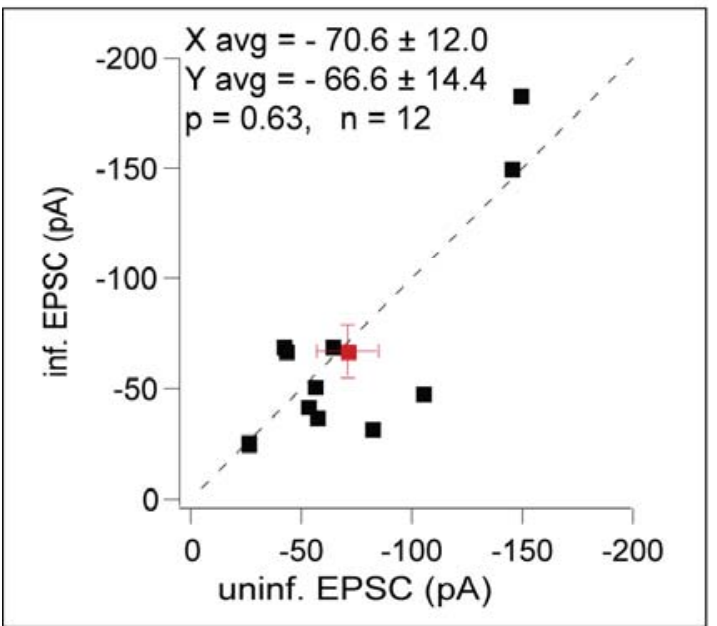

NMDAR EPSC

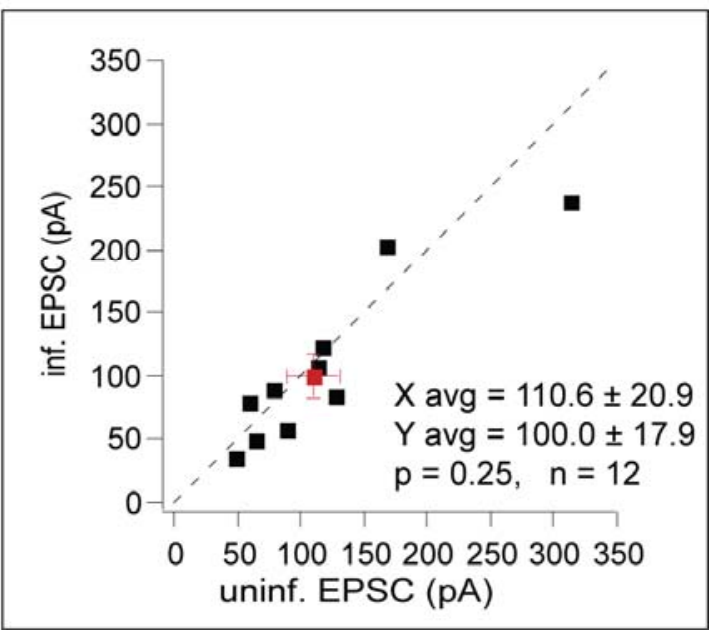

Figure 30. Effects of PSD-95 PDZ12 overexpression on basal synaptic transmission in PSD-95 KO mice.

A) Illustration depicting the PSD-95 PDZ12overexpressing vector. B) Dual-whole cell evoked EPSCs recorded from an untransfected control neuron and a PSD-95 PDZ12 overexpressing neighbor. Distributions show no significant change in AMPAR-EPSC amplitudes (left) nor in NMDAR-EPSC amplitudes (right). 


\section{III.B.2. Expression of full-length PSD-95 in PSD-95 KO neurons}

The next step was to assess if basal synaptic transmission can be rescued in PSD-95 KO neurons by expressing full-length PSD-95. The results show that expression of wild-type PSD-95 enhances the AMPAR-evoked current by 2.5 -fold, with no alteration in NMDAR-EPSCs (Figure 31). This demonstrates that AMPAR function can be rescued and enhanced by overexpression of full-length PSD-95 in PSD-95 KO mice; therefore showing that expression of full-length PSD-95 is functional in this experimental setting.

A.

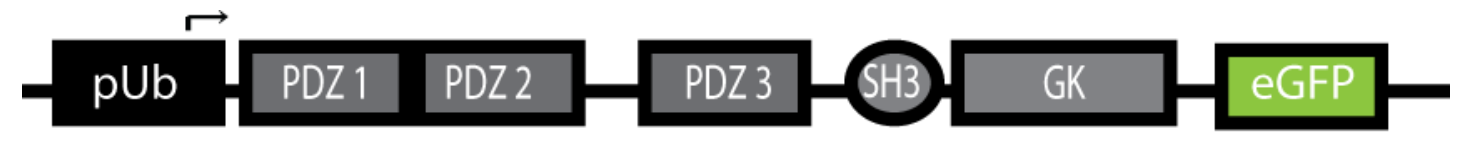

B.

AMPAR EPSC

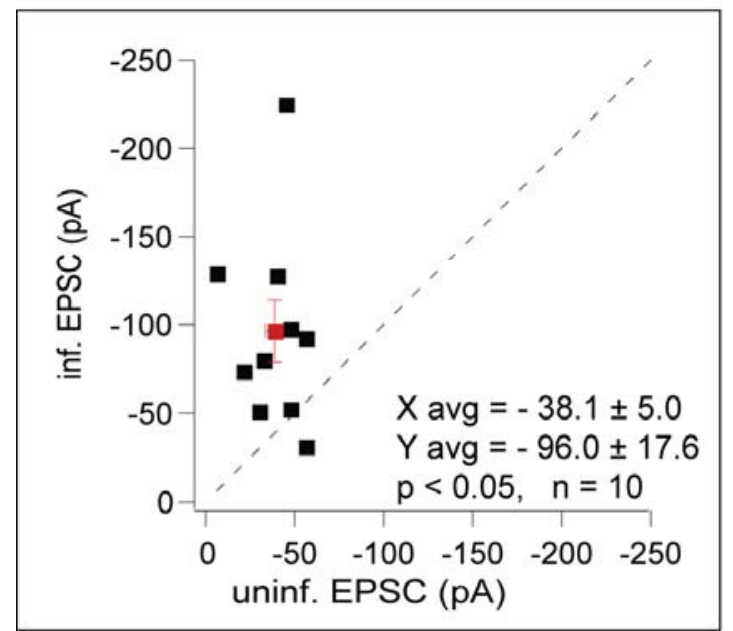

NMDAR EPSC

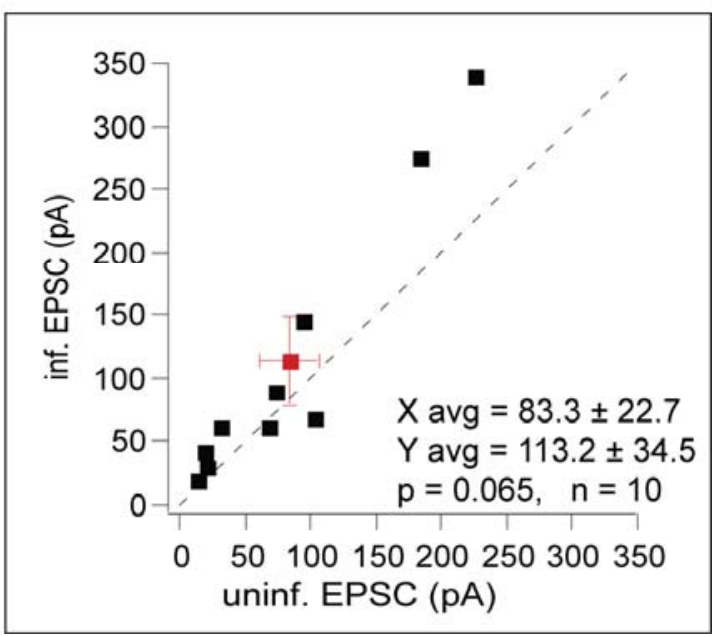


Figure 31. Effects of PSD-95 overexpression on basal synaptic transmission in PSD-95 KO mice.

A) Illustration depicting the overexpressing vector of full-length PSD-95. B) Dual-whole cell evoked EPSCs recorded from an untransfected control neuron and a wild-type PSD-95 overexpressing neighbor. Distributions show an increase in AMPAR-EPSC amplitudes (left) and no significant change in NMDAR-EPSC amplitudes (right).

\section{III.B.3. Expression of PSD-95 lacking its SH3 domain in PSD-95 KO neurons}

To test the requirement of the SH3 domain for PSD-95 for influencing basal synaptic transmission in the PSD-95 KO mouse, I performed dual whole cell recordings between a PSD-95 KO neuron (uninfected cell) and a neighboringinfected cell expressing PSD-95 $\Delta$ SH3. The results show that PSD-95 lacking its SH3 domain enhances AMPAR amplitudes by $\sim 2.2$ fold with no significant change on the NMDAR component (Figure 32). This result shows that PSD-95 $\Delta$ SH3 can enhance basal synaptic transmission as effectively as full-length PSD-95. This suggests that the SH3 domain of PSD-95 is not required for the protein to regulate synaptic strength.

A.

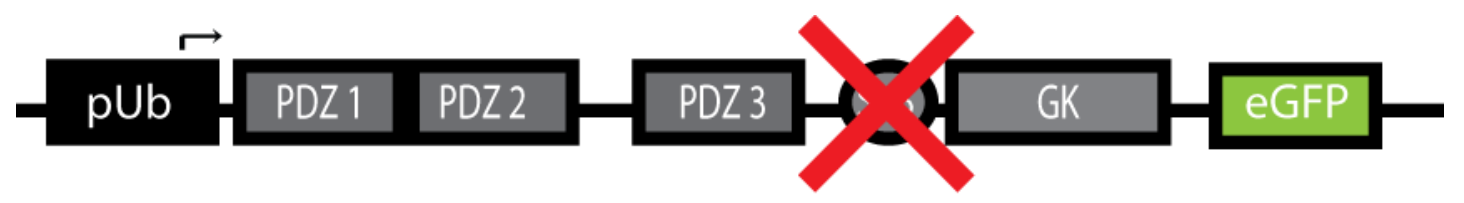

B. 
AMPAR EPSC

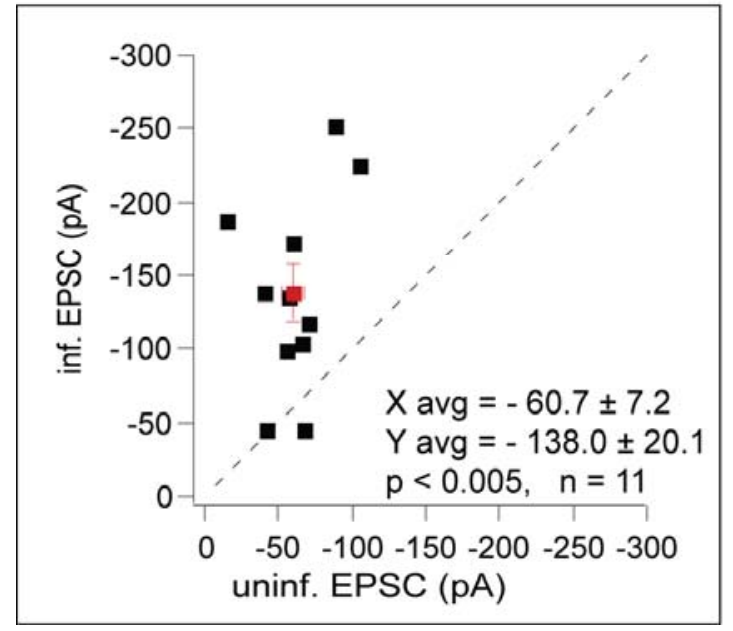

NMDAR EPSC

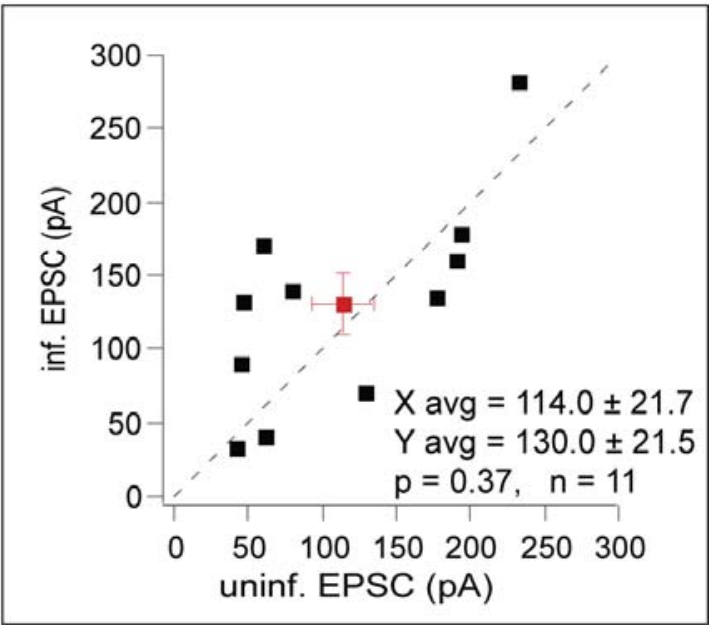

Figure 32. Effects of PSD-95 SH3 overexpression on basal synaptic transmission in PSD-95 KO mice.

A) Illustration depicting the overexpressing vector, PSD-95 $\Delta$ SH3. B) Dual-whole cell evoked EPSCs recorded from an untransfected control neuron and a PSD-95 $\Delta$ SH3 overexpressing neighbor. Distributions show an increase in AMPAR-EPSC amplitudes (left) and no significant change in NMDAR-EPSC amplitudes (right).

\section{III.B.4. Expression of PSD-95 lacking its GK domain in PSD-95 KO neurons}

A similar approach was adopted to test the requirement of the GK domain for PSD-95 to mediate its effects in basal synaptic transmission. Interestingly, expression of PSD-95 $\Delta \mathrm{GK}$ in PSD-95 KO neurons enhanced AMPAR function, similar to full-length PSD-95, without a significant change in NMDAR transmission (Figure 33). This experiment suggests that, in the absence of PSD95, the GK domain is dispensable for PSD-95 to regulate synaptic strength.

A.

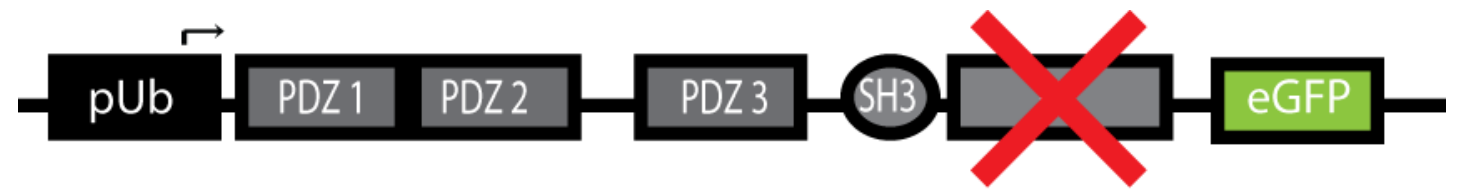

B. 
AMPAR EPSC

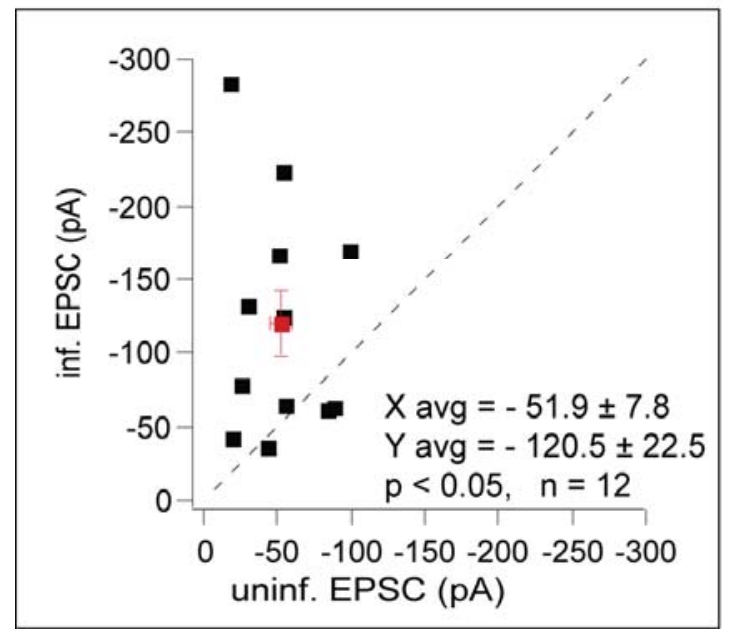

NMDAR EPSC

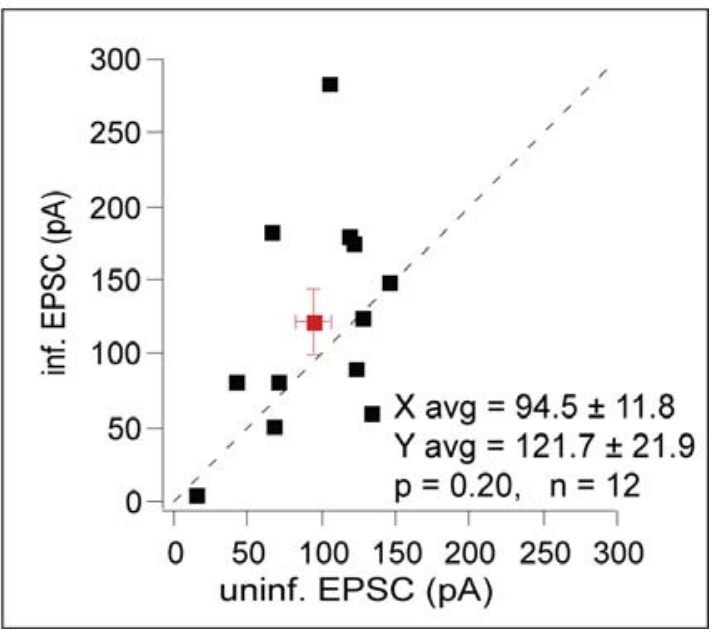

Figure 33. Effects of PSD-95 $\Delta$ GK overexpression on basal synaptic transmission in PSD-95 KO mice.

A) Illustration depicting the overexpression vector, PSD-95 $\Delta$ GK. B) Dual-whole cell evoked EPSCs recorded from an untransfected control neuron and a PSD-95 $\Delta$ GK overexpressing neighbor. Distributions show an increase in AMPAR-EPSC amplitudes (left) and no significant change in NMDAR-EPSC amplitudes (right).

\section{III.B.5. Expression of PSD-95 PDZ12-GK in PSD-95 KO neurons}

To further test the requirement of the GK domain in the process of basal synaptic transmission regulation mediated by PSD-95, I took yet another approach. It consisted in evaluating if adding the GK domain to the mutant expressing only PDZ12 (PDZ12 mutant which I showed to be non-functional in the absence of PSD-95 (Figure 30) could rescue and enhance synaptic transmission in the PSD-95 KO mouse. The results show that expressing PDZ12GK in PSD-95 KO neurons enhanced AMPAR-mediated transmission ( 1.8-fold), with no difference in NMDAR transmission (Figure 34). As PDZ12-GK is able to enhance AMPAR function in a similar way as full-length PSD-95, this result suggests a specific role of GK domain for PSD-95 to influence synaptic transmission.

A. 


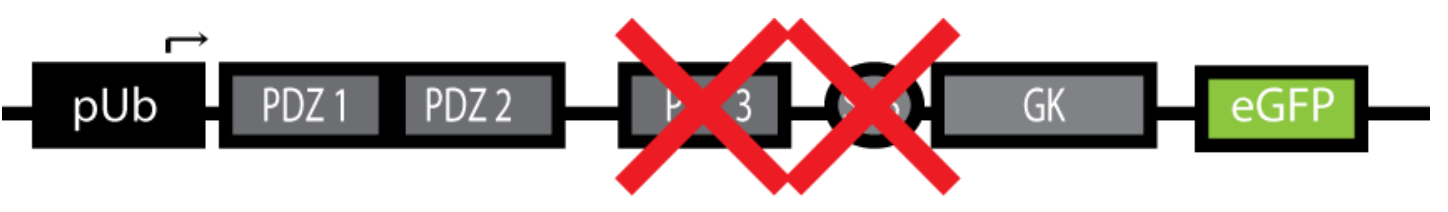

B.

AMPAR EPSC

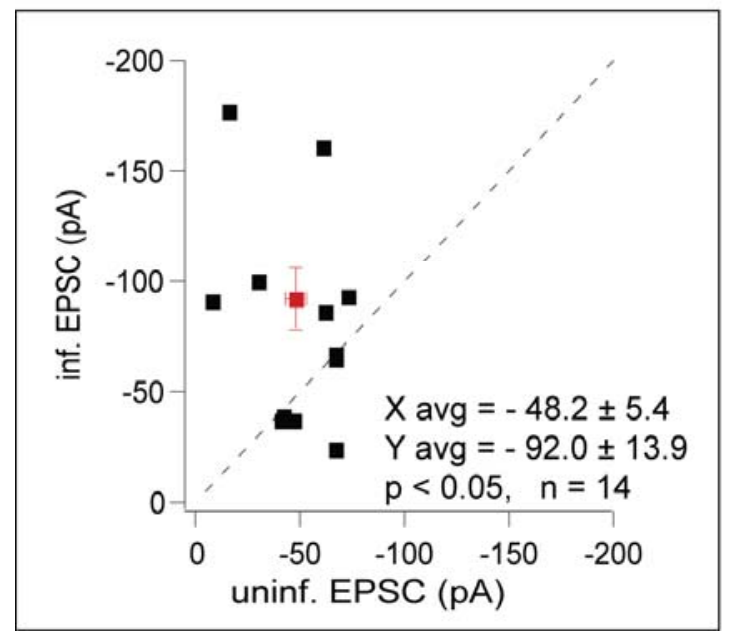

NMDAR EPSC

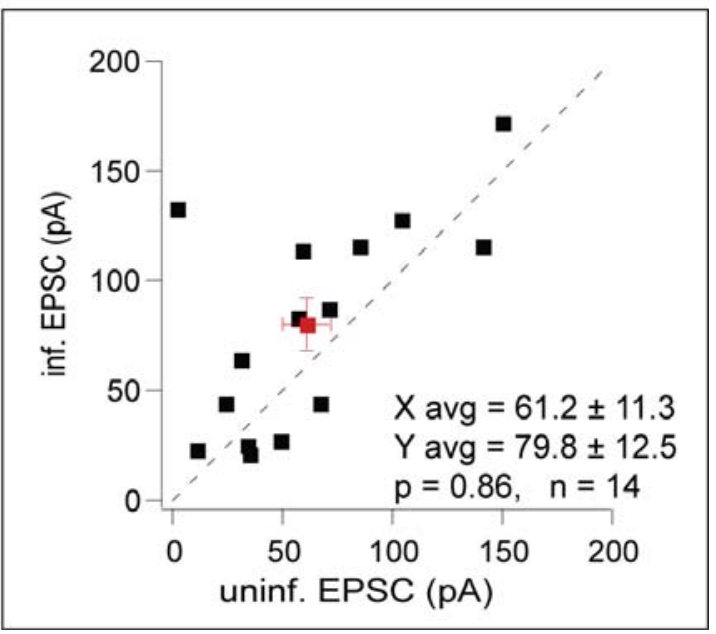

Figure 34. Effects of PSD-95 PDZ12-GK overexpression on basal synaptic transmission in PSD-95 KO mice.

A) Illustration depicting the overexpression vector, PSD-95 PDZ12-GK. B) Dual-whole cell evoked EPSCs recorded from an untransfected control neuron and a PSD-95 PDZ12-GK overexpressing neighbor. Distributions show an increase in AMPAR-EPSC amplitudes (left) and no significant change in NMDAR-EPSC amplitudes (right).

So far, these results obtained using PSD-95 KO mouse demonstrate that the effect of the PSD-95 mutants with C-terminal domains on rescuing and enhancing AMPAR function in the molecular replacement model is regardless of the residual endogenous PSD-95.

Moreover, they show that when one of the C-terminal domains is absent (PSD$95 \Delta$ SH3 or PSD-95 $\Delta$ GK experiments (Figure 32; Figure 33), the truncated protein is still functional. When both domains SH3 and GK are absent as well as PDZ3 (PSD95 PDZ12 experiment), this prevents the protein from rescuing and enhancing basal synaptic transmission (Figure 30). Furthermore, appending the GK domain to a non-functional PSD-95 (PDZ12) renders the mutant protein functional, as it 
is capable of enhancing synaptic strength as effectively as wild-type PSD95(Figure 34).

Altogether, these results obtained in the PSD-95 KO, suggest the requirement of one of the C-terminal domain, $\mathrm{SH} 3$ or $\mathrm{GK}$, in combination with the $\mathrm{N}$-terminal part including PDZ12 for PSD-95 to mediate its effect in regulating synaptic strength. In addition, they suggest a certain role mediated by the GK domain with respect to basal synaptic transmission.

These observations raised the question, why does a SH3 or GK domain seem to be necessary for the function, and why does either domain work? This might be explained by two factors:

- The increased levels of SAP102 and SAP97 at the post-synaptic density of the PSD-95 KO mouse (non-published data observed by T.Samaddar in our lab)

- And the ability of the MAGUK SH3 and GK domains to interact intermolecularly (Nix et al., 2000, McGee et al., 2001). Indeed, it was proposed that the SH3 domain of one DLG-MAGUK could interact with the GK domain of another DLG-MAGUK.

Based on these two factors, one can speculate that in the absence of PSD-95 (KO), the elevated level of SAP102 or SAP97 proteins enable an inter-molecular interaction with the overexpressed mutant of PSD-95. This inter-molecular interaction would rely on the SH3 or GK of SAP102 (or SAP97) interacting with the SH3 or GK domains of the truncated PSD-95. Therefore, the effect of the truncated PSD-95 constructs on synaptic transmission would be influenced by the elevation of SAP102 and/or SAP97 levels. 


\section{III.C. Molecular replacement studies in PSD-95 KO mouse hippocampal organotypic slice cultures}

\section{III.C.1. SAP102 knockdown combined with PSD- 95 $\triangle$ SH3 expression in PSD-95 KO neurons}

To test if SAP102 is participating in the rescue and enhancement effect mediated by the truncated forms of PSD-95, I used PSD-95 KO mouse organotypic slices combined with the molecular replacement approach, where SAP102 was knocked-down and PSD-95 mutant was expressed.

It is important to mention here that shRNA against SAP102 (sh102) has been shown to have no effect on basal synaptic transmission when recordings were done on late developmental stage (P15-P17) (Elias et al., 2008). That's why when I applied my experimental manipulations (sh102 combined to PSD-95 mutant expression and electrophysiological recording between P15 and P19), only an effect coming specifically from the interaction between SAP102 and the PSD-95 mutant should be observed (rather than an effect related to the SAP102 knockdown).

As a preliminary requisite, the SAP102 knockdown efficiency was tested in mouse dissociated cultures. Western blot results show a $80-90 \%$ decrease in the endogenous level of SAP102 (Figure 35). This validates the use of the shRNA against SAP102 to efficiently decrease the protein level.

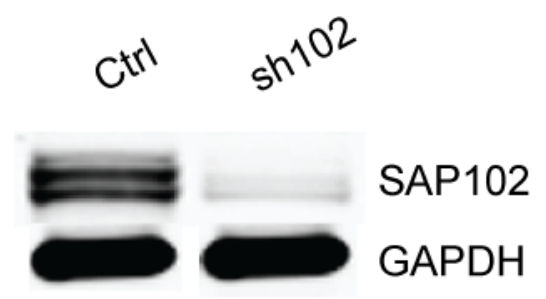

Figure 35. Acute knockdown, using short hairpin RNA against SAP102 (sh102) delivered by lentivirus. 
Sh102 resulted in an almost complete loss of endogenous SAP102 in dissociated mouse hippocampal neuronal cultures, probed by western-blot. GAPDH is used to normalize the signal to the amount of protein per lane.

In the first batch of recordings, I tested the role mediated by the SH3 domain of PSD-95 and SAP102 in the process of synaptic strength regulation, in the PSD-95 KO mouse. Knocking down SAP102 and expressing PSD-95 $\Delta$ SH3 in the PSD-95 KO mouse led to a 2.3 fold enhancement in AMPAR-evoked transmission with no alteration of the NMDAR-EPSC amplitude (Figure 36). This suggests that SAP102 is not involved in the AMPAR transmission enhancement mediated by PSD-95 $\Delta$ SH3 in the PSD-95 KO mouse.

A.

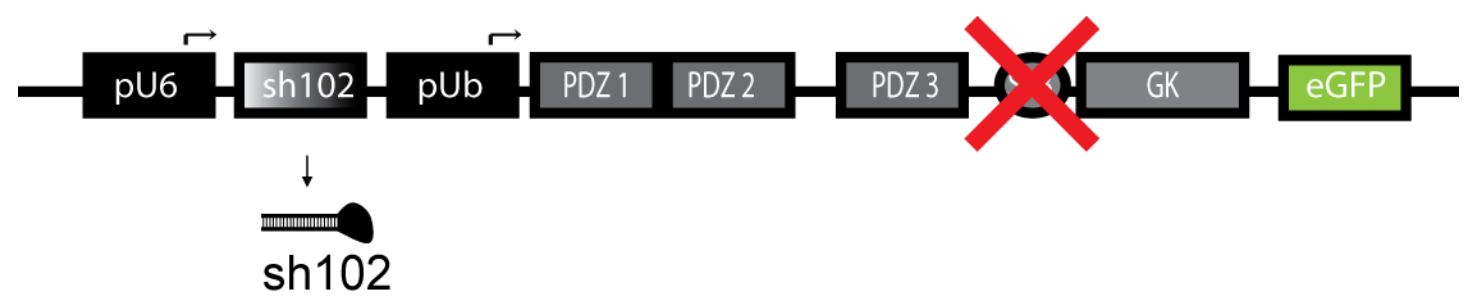

B. 
AMPAR EPSC

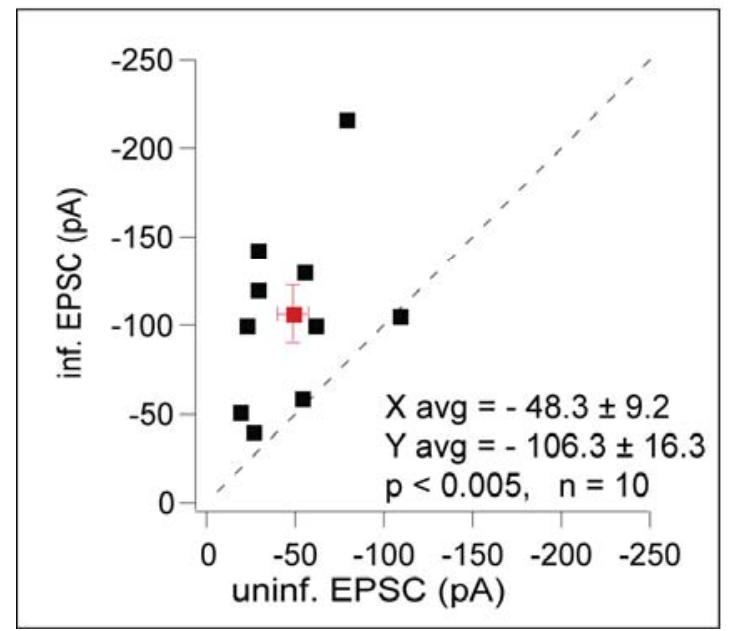

NMDAR EPSC

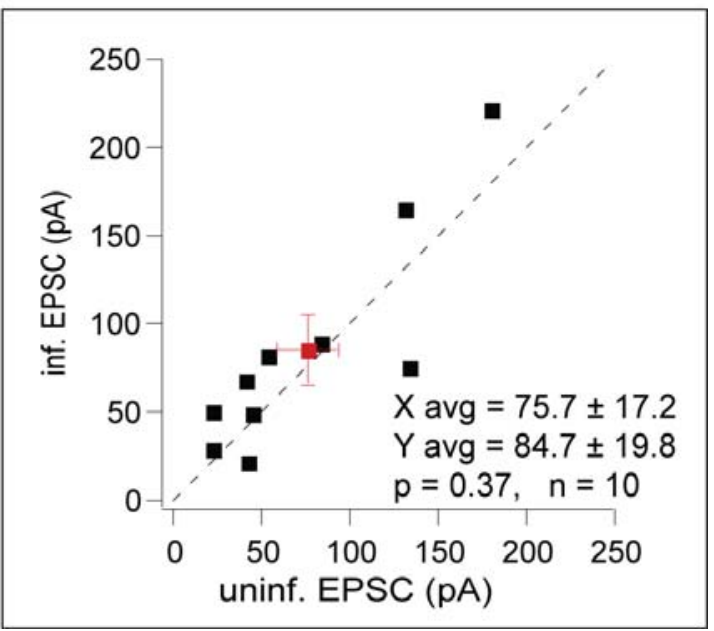

Figure 36. Effects of SAP102 knockdown combined with PSD-95 basal synaptic transmission in PSD-95 KO mice.

A) Illustration depicting the shRNA against SAP102 and replacement by PSD-95 $\Delta$ SH3 vector. B) Dual-whole cell evoked EPSCs recorded from an untransfected control neuron and a SAP102 knocking down expressing PSD-95 SSH3 neighbor in PSD-95 KO mice. Distributions show an increase in AMPAR-EPSC amplitudes (left) and no significant change in NMDAR-EPSC amplitudes (right).

\section{III.C.2. SAP102 knockdown combined with PSD- 95 $\Delta$ GK expression in PSD-95 KO neurons.}

In another set of recordings, I tested the role mediated by the GK domain of PSD-95, in combination with SAP102 to regulate synaptic strength in PSD-95 KO mice. SAP102 knockdown and expression of PSD-95 $\Delta$ GK in the PSD-95 KO failed to increase AMPAR-EPSCs, as both infected and control cells presented 
similar amplitudes of the AMPAR-component (Figure 37). This was accompanied by an increase in the NMDAR-component in the infected cell. This result suggests that the GK domain of PSD-95 is required in combination with SAP102 to mediate proper AMPAR-transmission in the PSD-95 KO mouse.

A.

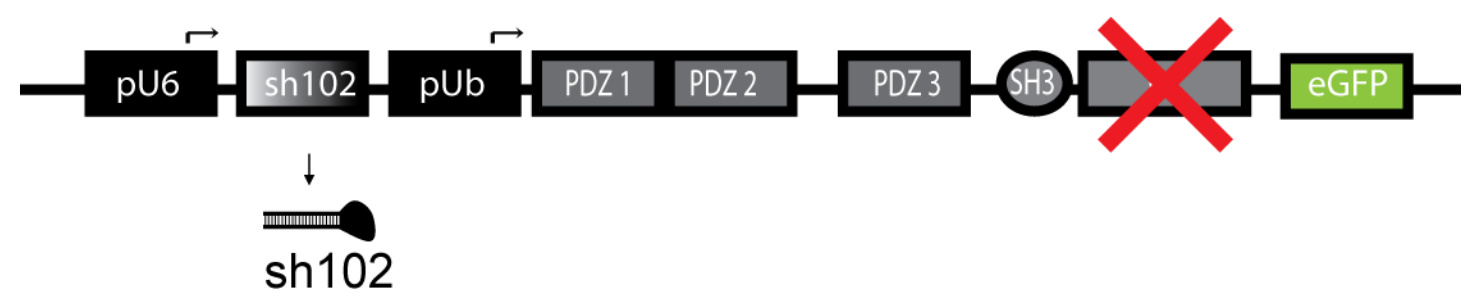

B.

AMPAR EPSC

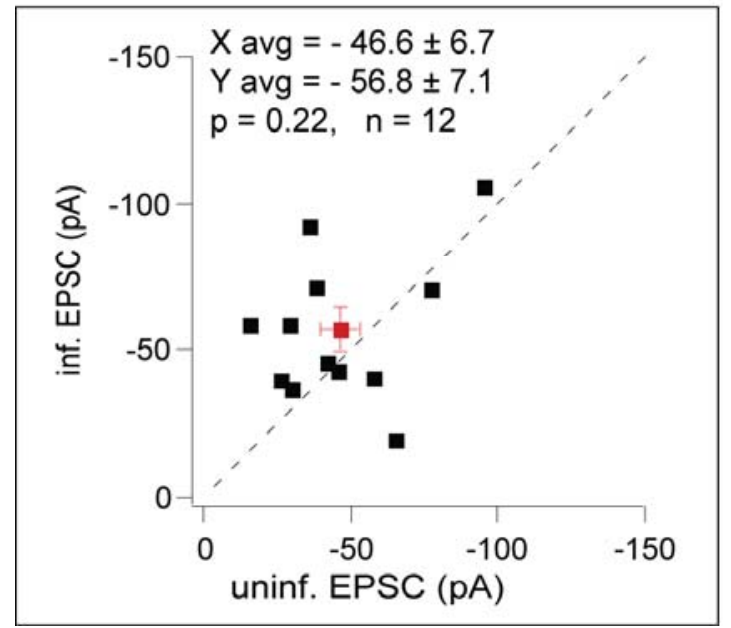

NMDAR EPSC

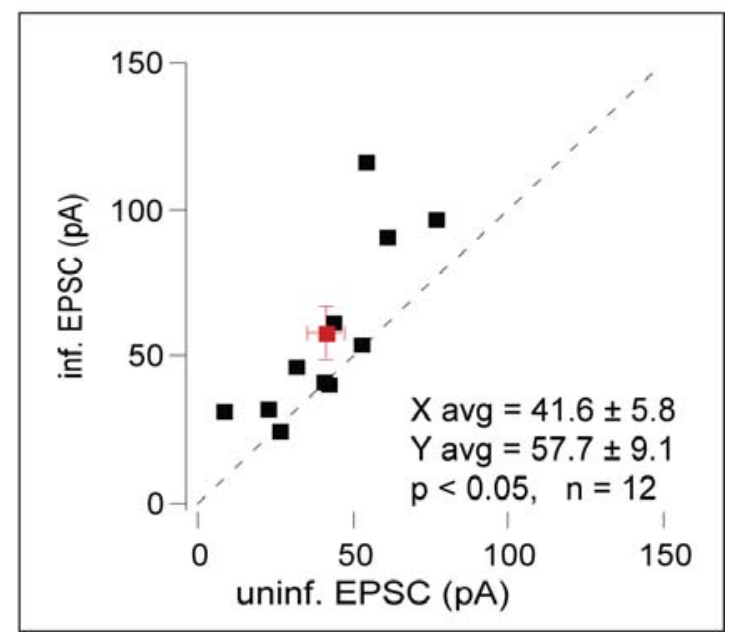

Figure 37. Effects of SAP102 knockdown combined with PSD-95 basal synaptic transmission in PSD-95 KO mouse.

A) Illustration depicting the vector expressing shRNA against SAP102 and replacement with PSD95 $\triangle$ GK. B) Dual-whole cell evoked EPSCs recorded from an untransfected control neuron and a neighboring SAP102 knockdown neuron simultaneously expressing PSD-95 $\Delta$ GK. Distributions show no significant change in AMPAR-EPSC amplitudes (left) and a small but significant increase in NMDAR-EPSC amplitudes (right).

\section{III.C.3. SAP102 knockdown combined with PSD-95 PDZ12-GK expression in PSD-95 KO neurons}


Another way of testing the requirement of the GK domain in combination with SAP102 was to knockdown endogenous SAP102 and express PSD-95 PDZ12-GK in PSD-95 KO mice. PDZ12-GK serves as a basis to check if the GK domain can restore the loss of function observed when PSD-95 PDZ12 is expressed. Electrophysiological recordings showed a 2.9 fold increase in AMPAR-evoked transmission with no alteration in the NMDAR-EPSC amplitude (Figure 38). This suggests that SAP102 is not crucial for PDZ12-GK to influence and enhance AMPAR transmission.

A.

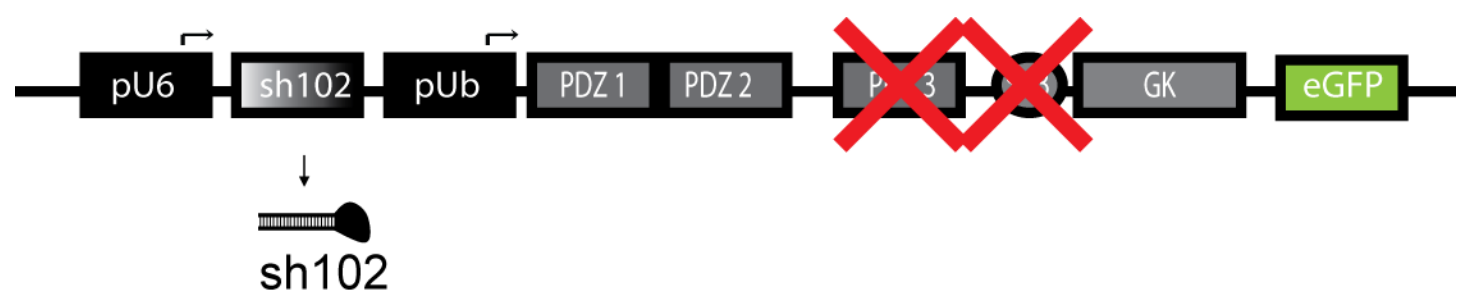

B.
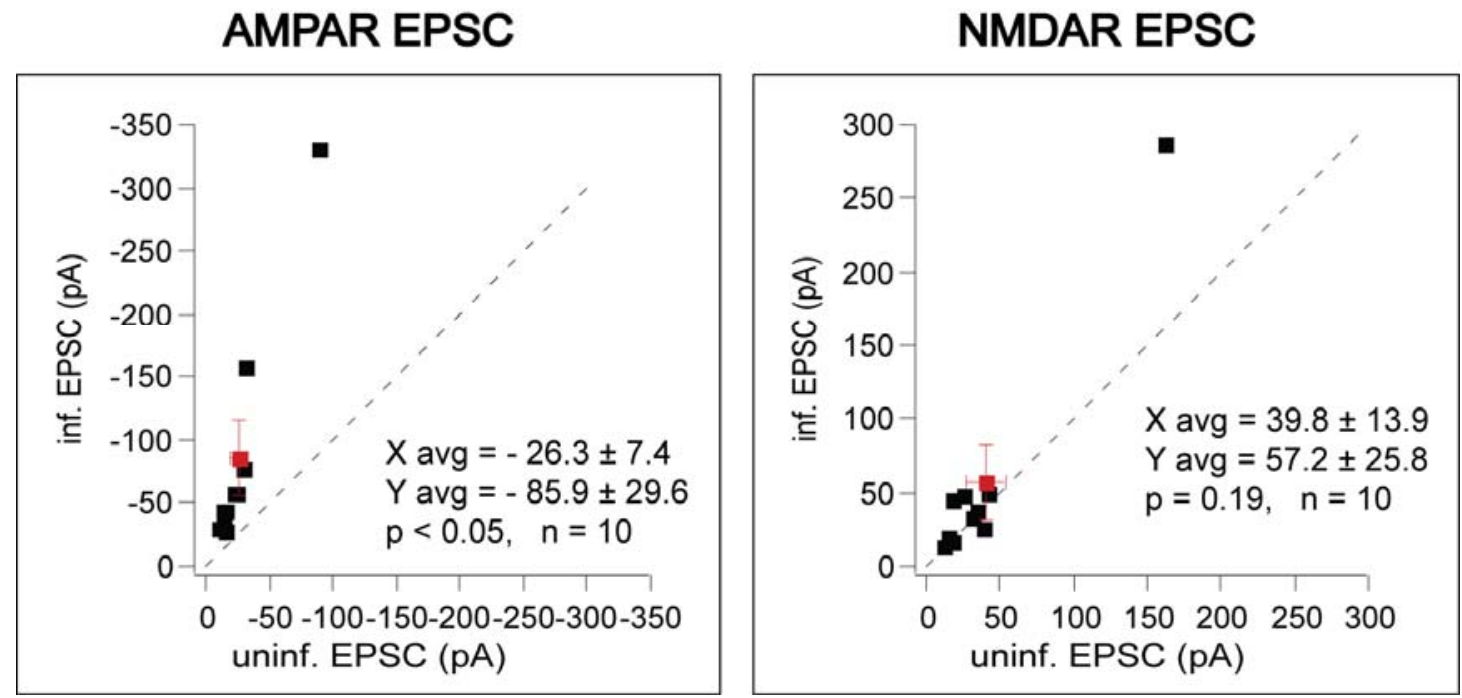

Figure 38. Effects of SAP102 knockdown combined with PSD-95 PDZ12-GK overexpression on basal synaptic transmission in PSD-95 KO mice.

A) Illustration depicting the vector expressing shRNA against SAP102 and replacement with PSD95 PDZ12-GK. B) Dual-whole cell evoked EPSCs recorded from an untransfected control neuron and a neighboring SAP102 knockdown neuron simultaneously expressing PSD-95 PDZ12-GK. Distributions show an increase in AMPAR-EPSC amplitudes (left) and no significant change in NMDAR-EPSC amplitudes (right). 


\section{Discussion}

In this study, I focused on elucidating the detailed molecular mechanism mediated by PSD-95, which influences AMPAR content at synapses. More precisely, I focused on understanding which domain(s) of PSD-95 are required for the protein to mediate its effect on basal synaptic transmission.

To assess the necessity of a specific domain of PSD-95, I created different PSD-95 mutants, which had various domain(s) of interest deleted, and then analyzed the functional properties of the mutants with respect to basal synaptic transmission. To this end, I used a combination of experimental approaches. On one hand, the molecular replacement technique in rat organotypic slice cultures and, on the other hand, the organotypic PSD-95 KO mouse slices cultures.

\section{IV.A. Validation of the approaches}

\section{IV.A.1. Domain deletion}

Deletion of specific domains of PSD-95, without affecting significantly the rest of the protein, is possible due to the modular structure of the protein (Feng and Zhang, 2009). This has been shown to work by different studies using this

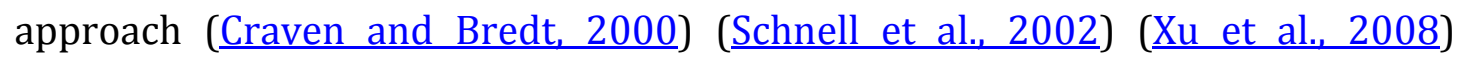
(Shipman et al., 2011), including this study. I was able to show that deletion of some domains leads to a loss-of-function, while deletion of others did not affect 
the function of the protein's affect on synaptic strength regulation (for example, Figure 13; Figure 15).

\section{IV.A.2. Molecular replacement}

In this study I first took advantage of the molecular replacement method. The molecular replacement manipulation relies on acute knockdown of the endogenous protein concomitant with the overexpression of the mutant protein under investigation. Therefore this technique relies on a restoration of a loss-offunction and, additionally, allows a restricted spatiotemporal manipulation. This has an advantage to limit developmental compensation, as the protein of interest is acutely knocked-down for a short period of time after the organism has already gone through critical developmental stages. Moreover, such an approach only targets a few cells, leaving the rest of the network intact, especially the neurons projecting on the analyzed one.

The molecular replacement approach used in this study could be validated since I could observe similar outcomes using molecular replacement and PSD-95 KO mouse.

Indeed, in both cases, expression of PSD-95 PDZ12 is not able to rescue basal synaptic transmission in the absence of PSD-95 (Figure 13, Figure 30 respeectively). Moreover, expression of wild-type PSD-95 in the molecular replacement model or in the total absence of PSD-95 (KO), leads to a rescue and increase of synaptic strength (Figure 11; Figure 31 respectively). In line with this, my results from the pure knockdown of PSD-95 (Figure 10) and from molecular replacement by wild-type PSD-95 (Figure 11) are in consistency with

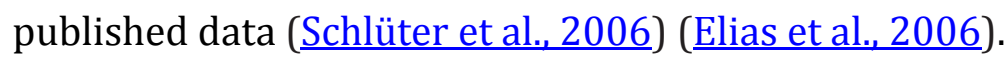




\section{IV.A.3. Validation of the mutants}

To differentiate between a loss-of-function of the mutant linked to the removal of specific domain or linked to the degradation of the mutant due to improper folding, the following experiment was performed. The mutant of interest was overexpressed in a wild-type neuron, and the effects were observed at the electrophysiological level. This experiment relies on the fact that PSD-95 has the capacity to multimerize with itself ( al., 1999). Thus, a functional mutant expressed in a wild-type neuron, will be able to enhance basal synaptic transmission. I performed this experiment with some PSD-95 mutants that were shown to partially rescue basal synaptic transmission, PSD-95 $\Delta$ GK and PSD-95 PDZ12-GK (Figure 24, Figure 26 respectively). I could show that these ambiguous mutants were able to elevate basal synaptic transmission, as effectively as full-length PSD-95, when expressed in the presence of endogenous PSD-95 (Figure 24, Figure 26 Figure 11 respectively). This experiment indicates that the functionality of these mutants was unaltered, thus validating their experimental use.

\section{IV.B. Domain requirement:}

\section{IV.B.1. PDZ3 or SH3 domains are not required for PSD-95 to mediate basal synaptic transmission}

From different experimental approaches (molecular replacement and/or PSD-95 KO), I found that PSD-95 lacking its PDZ3 domain or lacking its SH3 domain was still able to enhance AMPAR-evoked transmission in the absence of endogenous PSD-95 (Figure 15, Figure 17, Figure 32). These results suggest that each individual domain is dispensable from the protein to influence synaptic 
strength. In agreement with my results, Jo et al showed that in a molecular replacement context, PSD-95 SSH3 is able to rescue and enhance basal synaptic strength (Jo et al., 2010). This group utilized a similar approach as mine (molecular replacement and electrophysiology) with some minor differences. Namely, rat organotypic slice cultured at P6/7 combined with biolistic gene gun expression of mutant PSD-95 at 10-12 DIV, and electrophysiological dual whole cell patch-clamp recordings performed 3 to 4 days after transfection.

The fact that the SH3 domain can be removed from the protein without affecting its function on basal synaptic transmission suggests that some molecular pathways related to the SH3 binding partners might be negligible for regulating basal synaptic strength.

For example, hippocalcin is a protein that binds specifically to the SH3 domain of PSD-95 (Lo et al., 2010). Hippocalcin has been shown to play a role in long term depression of NMDAR EPSCs induced by muscarinic receptors (Jo et al., 2010). Based on my result, hippocalcalcin does not seem to be involved in the regulation of synaptic strength mediated by PSD-95.

The tyrosine kinase Pyk2, has also been shown to interact with the SH3 domain of PSD-95 ( Seabold, 2003). Its binding to the domain seems to be required for clustering and activating Pyk2 during long term potentiation mediated by NMDARs at CA3-CA1 synapses (Bartos et al., 2010). Hence, Pyk2 does not seem to participate in the regulation mediated by PSD-95 to influence basal synaptic transmission.

The involvement of hippocalcin and Pyk2 seems to be related to plasticity pathways, which is why they might not be involved in the regulation of basal synaptic transmission. This is in agreement with the fact that PSD-95's role in regulating synaptic strength can be molecularly dissociated from its role in plasticity ( $\underline{\text { Xu et al., 2008). }}$. 
The SH3 domain of PSD-95 has also been shown to interact with EB3 (Sweet et $\mathrm{al}_{\text {., 2011) }}$. EB3 is a protein involved in dendrite branching. The observation that disrupting the PSD-95-EB3 binding does not affect synaptic strength correlates with the fact that dendrite branching is prominent during neuron development. Therefore, at the time point where I introduce PSD-95 lacking the SH3 domain to the neurons, normal dendritic arborization had already taken place, and normal synaptic transmission can be conducted.

Moreover, as synaptic strength is not negatively influenced by the block of PSD-95/EB3 pathway, this suggests that the PSD-95 SH3-EB3 interaction might be important for dendritic development, associated with synaptic strengthening, but not for the maintenance of dendritic branches.

One mechanism to strengthen synaptic transmission is to convert silent synapse to a functional synapses (silent synapses are synapses that exhibit NMDAR mediated response, but no AMPAR-mediated response). Interestingly, PSD-95 has been shown to play a role in unsilencing AMPAR silent synapses (Stein et al., 2003). Therefore, the fact that blocking the PSD-95 EB3 pathway in late development is not affecting basal synaptic transmission is consistent with the fact that, at this time, further synapses number (synaptic strengthening) and/or dendritic branches are not as much needed as during synaptogenesis for wiring the network.

Concerning the PDZ3 signaling pathway, some functional interactions do not appear to contribute to PSD-95's influence on synaptic transmission.

DHCC5 is a newly identified palmitoyltransferase protein enriched in the post synaptic part of a neuron which has been shown to interact with the PDZ3 domain of PSD-95 (Li et al., 2010). Its role is unclear, but DHCC5 was suggested to play a role in hippocampal learning due to the impairment of contextual fear conditioning. It is suggested that by binding to PSD-95, DHCC5 could localize next to important signaling molecules, such as nNOS (neuronal Nitric Oxide Synthase), and thus influence learning processes. However, as basal synaptic 
transmission is maintained when PSD-95 lacks its PDZ3 domain, DHCC5 does not seem to take part in the molecular pathway mediated by PSD-95 to influence basal synaptic strength.

Murata et al showed that PRR7, a brain enriched proline-rich membrane protein, also binds the PDZ3 domain of PSD-95 (Murata et al., 2005). Unfortunately, the function of PRR7 is yet unclear. But according to my results, PRR7 does not seem to be involved in molecular pathways related to synaptic strength regulation mediated by PSD-95.

Neuroligins are another ligands type that binds the PDZ3 domain of PSD95 (Irie et al., 1997). Due to the absence of effect in basal synaptic transmission while removing the PDZ3 domain of PSD-95, this suggests that the neuroligin/PDZ3 PSD-95 pathway is not involved in the regulation of basal synaptic strength.

\section{IV.B.2. GK domain is required for PSD-95 to mediate basal synaptic transmission, when SAP102 does not compensate}

The first approach taken to evaluate the role of the GK domain on PSD-95 to influence synaptic strength was to delete the GK domain and assess the consequences at the electrophysiological level. Deleting the GK domain from over-expressed PSD-95 in molecular replacement experiments only partially rescued basal synaptic strength to the control the cell level (Figure 19).

The second approach taken to evaluate the role of the GK domain with respect to basal synaptic transmission was to use a mutant protein known to be nonfunctional (PSD-95 PDZ12) and to attach the GK domain to it. The aim was to evaluate if the GK domain could rescue the loss-of-function caused by PSD-95 
PDZ12. In this scenario, a partial rescue to the control cell level was also observed (Figure 22).

As already discussed (paragraph IV.A.3), this partial rescue does not seem linked to a non-functional mutant proteins, as overexpression of each mutant (PSD-95 $\Delta$ GK and PSD-95 PDZ12-GK) significantly enhanced AMPAR transmission (Figure 24, Figure 26), and thus behaves similar to a full-length and fully functional PSD-95 with respect to basal synaptic transmission.

The expression level of PSD-95 $\Delta$ GK was also questioned as possible origin mediating the partial rescue (Figure 21). However, differences in the expression levels are highly unlikely because another mutant (PSD-95 PDZ12-GK), which also led to a partial rescue, was strongly expressed (Figure 22).

While repeating this experiment (molecular replacement by PSD-95 $\Delta$ GK), I could observe differences in the results, ranging from partial rescue (as seen at first (Figure 19) to an enhanced basal synaptic transmission (Figure 28). These differences were neither related to the differences between the overall activities of each slices culture (Figure 29), nor dependent on the time when recordings were performed (e.g., soon after virus injection or late after virus injection) (data not shown).

This first approach (molecular replacement) points out the necessity of the GK domain to some extent. When removed, the protein mediates only a partial effect. Also, when added to a non-functional protein, this new mutant mediates only a partial recovery of synaptic strength compared to control cells.

The last question concerning the partial rescue was related to the endogenous levels of PSD-95. As already mentioned, this is a crucial point, as it

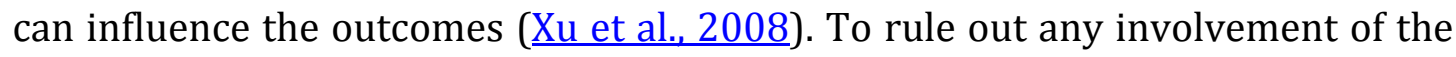
endogenous PSD-95 in the molecular replacement using PSD-95 $\Delta$ GK or PSD-95 
PDZ12, I took advantage of another approach: overexpressing a mutant protein in PSD-95 KO mouse background. In this case, both PSD-95 $\Delta$ GK and PSD-95 PDZ12-GK showed an increase in AMPAR-EPSC amplitude (Figure 33, Figure 34, respectively) similar to the full-length PSD-95 (Figure 31). These results show that the previous results observed in the molecular replacement (PSD-95 GKK and PSD-95 PDZ12-GK) were not biased by endogenous PSD-95. Moreover, these experiments performed in the PSD-95 KO mouse, on one hand, point to the nonrequirement of the GK domain (PSD-95 GKK experiment; Figure 33). But on the other hand, point to the involvement of the GK domain (PSD-95 PDZ12-GK experiment; Figure 34,) on PSD-95 in influencing basal synaptic strength.

The non-requirement of the GK domain is supported by a recent study from Sturgill et al. 09 (Sturgill et al., 2009) where 2-photon laser photoactivation imaging was combined with a molecular replacement approach. It is shown that when PSD-95 is knocked-down and replaced by PSD-95 $\Delta$ SH3-GK, the mutant is less stable at the synapse $(\sim 50 \%$ of GFP-tagged PSD-95 $\Delta$ SH3-GK remaining at the synapse 30 minutes after the photo-bleaching pulse). But, when the PSD-95 knockdown is combined with PSD-95 GGK expression, the mutant protein appears to be as stable as the full length PSD-95 (90\% of GFP-tagged PSD-95 $\Delta$ GK remaining at the synapse 30 minutes after the photo-bleaching pulse). While this study focused only on the localization of the mutants, my approach is additionally investigating the function of each mutant. Nonetheless, combined together, the results support the non-requirement of the GK domain for PSD-95 for maintaining its stability at the synapse when only PSD-95 is decreased.

Moreover, Jo and co-workers, by using a similar design to the current study, showed that molecular replacement by PSD-95 $\Delta$ GK in rat organotypic slices led to an increase in basal synaptic transmission, similar to the increase observed with full-length PSD-95 (Jo et al., 2010). These results, in combination 
with mine, support the non-requirement of the GK domain when only PSD-95 is absent.

Although the results concerning the role mediated by GK domain are sometimes similar between the two approaches (molecular replacement and use of PSD-95 KO), in showing an increase in AMPAR transmission, sometimes only a partial rescue in basal synaptic transmission was observed using the molecular approach (Figure 28). This might be explained by functional compensation by others DLG-MAGUK family members, such as SAP97 or SAP102. This hypothesis is supported by an elevated level of SAP102 and SAP97 at the postsynaptic density of PSD-95 KO mice (non-published data observed by T.Samaddar in our lab).

The functional compensation hypothesis might reconcile the different results obtained when GK domain is either deleted from PSD-95 (PSD-95 GK) or appended to a non-functional mutant of PSD-95 (PSD-95 PDZ12-GK). As both experiments suggest opposite result (non requirement for the former, requirement for the later). A functional compensation, associated to the fact that different DLG-MAGUK interact among each other using their SH3 GK domains, might explain these opposite results. Taking into consideration that SH3 and GK domains have been suggested to interact inter-molecularly, between one DLGMAGUK and another (Nix et al., 2000) (McGee et al., 2001), this would explain why deleting the GK domain from PSD-95 still allows PSD-95 to enhance basal synaptic transmission as efficiently as full-length PSD-95. In this scenario (PSD95 $\Delta \mathrm{GK})$, PSD-95 could use its SH3 domain to bind to the GK domain of SAP102 or SAP97 and thus still maintain normal synaptic strength. Vice versa, the presence of the GK domain of PSD-95 (PSD-95 PDZ12-GK scenario) would allow it to interact with the SH3 domain of SAP102, and thus still elevate synaptic strength.

The functional interaction between SAP102 and the GK domain of PSD-95 was indeed involved in the rescue of basal synaptic transmission, as knocking 
down SAP102 and overexpressing PSD-95 GGK failed to rescue and enhance synaptic strength in the PSD-95 KO (Figure 37). In addition, this was not observed with the PSD-95 $\Delta$ SH3 mutant (Figure 36). Importantly, knocking down SAP102 in slice cultures from P7 animals did not influence basal synaptic transmission (Elias et al., 2008). Firstly, this experiment shows the importance of the GK domain for PSD-95 to regulate basal synaptic transmission.

Secondly, in the PSD-95 KO, the comparison of the results

1. overexpressing PSD-95 $\Delta$ GK (increase in basal synaptic strength) and

2. overexpressing PSD-95 $\Delta$ GK while knocking SAP102 (no increase in basal synaptic strength),

shows that SAP102 can mask the effect mediated by PSD-95 $\Delta \mathrm{GK}$, and thus compensate to mediate proper basal synaptic transmission.

Finally, knocking down SAP102 and overexpressing PSD-95 PDZ12-GK was still able to rescue and enhance synaptic strength in the PSD-95 KO (fig). This experiment emphasizes the important role mediated by GK domain for PSD95 to influence basal synaptic transmission.

In accordance with the importance of the GK domain, The GK domain links PSD-95 to GKAP/SAPAP (Takeuchi et al., 1997) (Kim et al., 1997) (Kawashima and Sobue, 1997), and Shanks (Naisbitt et al., 1999), building the core structure of the PSD (Hayashi et al., 2009). Not only binding to GKAP/SAPAP, GK domain of PSD-95 has also been shown to interact with MAP1 (Reese et al., 2007), S-CAM (

The most tempting speculation concerns the involvement of GKAP/SAPAP proteins as it might link PSD-95 to the actin cytoskeleton and promote morphological and functional changes at the synapses. 


\section{IV.C. Differences in the maturational state of synapses between acutely knocked-down PSD- 95 and germline removal of PSD-95}

When PSD-95 PDZ12-GK was analyzed in the context of molecular replacement, this mutant protein was only able to rescue basal synaptic transmission to the control cell level (Figure 22). But when analyzed in PSD-95 KO neurons, it was able to rescue and enhance synaptic strength (Figure 34) as efficiently as the full-length PSD-95. The difference between the two approaches resides in the time that PSD-95 is absent.

According which approaches was taken, molecular replacement versus PSD-95 KO mouse slices culture, a more mature synapse was under investigation.

In the molecular replacement approach, normal maturation processes have had time to take place, as PSD-95, which is known to be also involved in the synaptic maturation of excitatory synapses (El-Husseini et al., 2000b), was only knocked-down beginning on 2DIV after P8, and was done so among an intact network of neurons. In contrary, in the PSD-95 KO mouse, PSD-95 was never present. As such, a synapse investigated with the molecular replacement approach can be considered as more mature than a synapse in the PSD-95 KO mouse.

In immature synapses, PSD-95 PDZ12-GK seems to be enough to anchor itself into the postsynaptic density and enhance synaptic transmission, while PSD95 $\Delta$ GK might do so by interaction with SAP102.

In mature synapses, where PSD-95 is the most abundant DLG-MAGUK, PSD-95 PDZ12-GK might not be sufficient and either SH3 or PDZ3 domains are additionally required. The lack of GK also diminishes the function (partial rescue). 
Therefore, the effect of the mutant seems related to the maturational stage of the synapse. Some aspects of an immature synapse seems providing the ground for the mutants to perform their function in basal synaptic transmission.

One of the potential features of a more immature synapse in the PSD-95 KO mouse is its ability to trigger compensational mechanisms. These compensatory mechanisms might be restricted in the molecular replacement experiments due to time limitations. That is likely why, in the PSD-95 KO, PSD-95 $\Delta$ GK was able to enhance basal synaptic transmission (Figure 33), while it became more difficult in the molecular replacement context (Figure 19) as compensation might not had time to occur.

\section{IV.D. The NMDAR component}

In this study, I could observe a moderate increase in NMDA-EPSC amplitude (Figure 12, Figure 17, Figure 28) while in some cases, which is in contrast to previous work showing specific regulation of AMPAR-mediated

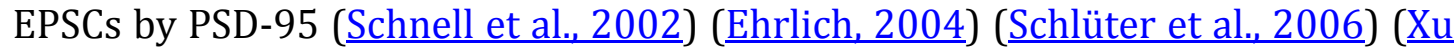
et al., 2008) (Elias et al., 2008). However, such NMDAR-EPSC amplitude increases have also been seen by other groups (Kim et al., 2007) (Futai et al., 2007). Such differences might derive from factors such as the age of the animals, beginning, duration, and efficacy of altering PSD-95 levels, or the number of manipulated neurons in the network.

The variable effect of different PSD-95 mutants on the NMDARcomponent does not seem to be related to an increase in AMPAR-transmission. Molecular replacement by PSD-95 $\Delta$ SH3 led to a 1.9-fold augmentation of AMPAR-EPSC amplitude, accompanied by a 1.3-fold increase in NMDAR-EPSC amplitude, while molecular replacement by PSD-95 PDZ3 led to a 2.2-fold 
enhancement in AMPAR with no change in NMDAR transmission. Interestingly, a trans-synaptic signaling and enhancement of presynaptic function has been shown to involve PSD-95 at excitatory synapses (Futai et al., 2007). Such a scenario could explain the increase in both AMPAR and NMDAR transmission.

\section{IV.E. Conclusion and outlook}

Taking a combined approach, utilizing molecular replacement and PSD-95 KO mice, I was able to show that PSD-95 does not require its PDZ3 domain or SH3 domain but rather rely on its GK domain (in combination with the PDZ12 domains) to regulate basal synaptic transmission. Moreover, this function of the GK domain can be masked by SAP102. Therefore, when SAP102 can compensate (by an elevated level for example), GK domain seems dispensable for PSD-95 to regulate basal synaptic strength. Therefore, specific interacting partners of the GK domain might be involved in the molecular pathway leading to the tight regulation of basal synaptic transmission mediated by PSD-95. The most tempting speculation involves the SPAR or GKAP/SAPAP molecular pathways, as these proteins have been shown to belong to the structural core of the postsynaptic density.

In the future, it will be interesting to decipher more precisely which interacting partners of the GK domain are involved in the process of synaptic strength regulation. Is it exclusively the interaction with SPAR that is required, or is it the one with GKAP/SAPAP? Or, are other GK domain interacting partners also involved? Single point mutations can be used to specially interrupt some proteinprotein interactions and answer to the above listed questions. Understanding the basis of basal synaptic transmission seems a critical step as PSD-95 has been shown to be involved in some neurological disorders (Kristiansen et al., 2006). 


\section{REFERENCES CITED}

A. L. Shapiro EV, and J. V. Maizel. (1967) Molecular weight estimation of polypeptide chains by electrophoresis in SDS-polyacrylamide gels. Biochem Biophys Res Commun 28:815-820.

Adesnik H, Nicoll RA, England PM (2005) Photoinactivation of native AMPA receptors reveals their real-time trafficking. Neuron 48:977-985.

Ashby MC, Maier SR, Nishimune A, Henley JM (2006) Lateral diffusion drives constitutive exchange of AMPA receptors at dendritic spines and is regulated by spine morphology. Journal of Neuroscience 26:7046-7055.

Ayalon G, Stern-Bach Y (2001) Functional assembly of AMPA and kainate receptors is mediated by several discrete protein-protein interactions. Neuron 31:103-113.

Banke TG, Bowie D, Lee H, Huganir RL, Schousboe A, Traynelis SF (2000) Control of GluR1 AMPA receptor function by cAMP-dependent protein kinase. Journal of Neuroscience 20:89-102.

Barria A, Muller D, Derkach V, Griffith LC, Soderling TR (1997) Regulatory phosphorylation of AMPA-type glutamate receptors by CaM-KII during long-term potentiation. Science 276:2042-2045.

Bartos JA, Ulrich JD, Li H, Beazely MA, Chen Y, Macdonald JF, Hell JW (2010) Postsynaptic clustering and activation of Pyk2 by PSD-95. The Journal of neuroscience : the official journal of the Society for Neuroscience 30:449463.

Bats C, Groc L, Choquet D (2007) The interaction between Stargazin and PSD-95 regulates AMPA receptor surface trafficking. Neuron 53:719-734.

Baughman JEHaRW (1986) Primary culture of identified neurons from the visual cortex of postnatal rats. J Neurosci 6:3044-3060.

Béïque J-C, Lin D-T, Kang M-G, Aizawa H, Takamiya K, Huganir RL (2006) Synapse-specific regulation of AMPA receptor function by PSD-95. Proceedings of the National Academy of Sciences of the United States of America 103:19535-19540.

Blanpied TA, Scott DB, Ehlers MD (2002) Dynamics and regulation of clathrin coats at specialized endocytic zones of dendrites and spines. Neuron 36:435-449.

Bliss TV, Lomo T (1973) Long-lasting potentiation of synaptic transmission in the dentate area of the anaesthetized rabbit following stimulation of the perforant path. The Journal of physiology 232:331-356.

Borgdorff AJ, Choquet D (2002) Regulation of AMPA receptor lateral movements. Nature 417:649-653.

Braithwaite SP, Meyer G, Henley JM (2000) Interactions between AMPA receptors and intracellular proteins. Neuropharmacology 39:919-930. 
Bredt DS, Nicoll RA (2003) AMPA receptor trafficking at excitatory synapses. Neuron 40:361-379.

Brenman JE, Christopherson KS, Craven SE, McGee AW, Bredt DS (1996) Cloning and characterization of postsynaptic density 93, a nitric oxide synthase interacting protein. The Journal of neuroscience : the official journal of the Society for Neuroscience 16:7407-7415.

Bridgman PC (2004) Myosin-dependent transport in neurons. Journal of neurobiology 58:164-174.

Buckby LE, Mummery R, Crompton MR, Beesley PW, Empson RM (2004) Comparison of neuroplastin and synaptic marker protein expression in acute and cultured organotypic hippocampal slices from rat. Brain research Developmental brain research 150:1-7.

Burnashev N, Villarroel A, Sakmann B (1996) Dimensions and ion selectivity of recombinant AMPA and kainate receptor channels and their dependence on Q/R site residues. The Journal of physiology 496 ( Pt 1):165-173.

Carlisle HJ, Fink AE, Grant SGN, O\&apos;Dell TJ (2008) The ups and downs of synaptic plasticity: influences on this particular \&apos;market\&apos; pas ca The Journal of physiology 586:5885-5900.

Carroll RC, Beattie EC, von Zastrow M, Malenka RC (2001) Role of AMPA receptor endocytosis in synaptic plasticity. Nature Reviews Neuroscience 2:315324.

Carroll RC, Beattie EC, Xia H, Lüscher C, Altschuler Y, Nicoll RA, Malenka RC, von Zastrow M (1999) Dynamin-dependent endocytosis of ionotropic glutamate receptors. Proceedings of the National Academy of Sciences of the United States of America 96:14112-14117.

Chen L, Chetkovich DM, Petralia RS, Sweeney NT, Kawasaki Y, Wenthold RJ, Bredt DS, Nicoll RA (2000) Stargazin regulates synaptic targeting of AMPA receptors by two distinct mechanisms. Nature 408:936-943.

Chen X, Vinade L, Leapman RD, Petersen JD, Nakagawa T, Phillips TM, Sheng M, Reese TS (2005) Mass of the postsynaptic density and enumeration of three key molecules. Proceedings of the National Academy of Sciences of the United States of America 102:11551-11556.

Cheng D, Hoogenraad CC, Rush J, Ramm E, Schlager MA, Duong DM, Xu P, Wijayawardana SR, Hanfelt J, Nakagawa T, Sheng M, Peng J (2006) Relative and absolute quantification of postsynaptic density proteome isolated from rat forebrain and cerebellum. Molecular \&amp; cellular proteomics : MCP 5:1158-1170.

Chetkovich DM, Bunn RC, Kuo S-H, Kawasaki Y, Kohwi M, Bredt DS (2002) Postsynaptic targeting of alternative postsynaptic density-95 isoforms by distinct mechanisms. Journal of Neuroscience 22:6415-6425.

Cho C-H, St-Gelais F, Zhang W, Tomita S, Howe JR (2007) Two families of TARP isoforms that have distinct effects on the kinetic properties of AMPA receptors and synaptic currents. Neuron 55:890-904.

Cho KO, Hunt CA, Kennedy MB (1992) The rat brain postsynaptic density fraction contains a homolog of the Drosophila discs-large tumor suppressor protein. Neuron 9:929-942. 
Collingridge GL, Olsen RW, Peters J, Spedding M (2009) A nomenclature for ligand-gated ion channels. Neuropharmacology 56:2-5.

Craven SE, Bredt DS (2000) Synaptic targeting of the postsynaptic density protein PSD-95 mediated by a tyrosine-based trafficking signal. The Journal of biological chemistry 275:20045-20051.

Craven SE, El-Husseini AE, Bredt DS (1999) Synaptic targeting of the postsynaptic density protein PSD-95 mediated by lipid and protein motifs. Neuron 22:497-509.

D. Muller PAB, and L. Stoppini (1993) Time course of synaptic development in hippocampal organotypic cultures. Brain Res Dev Brain Res 71:93-100.

Dakoji S, Tomita S, Karimzadegan S, Nicoll RA, Bredt DS (2003) Interaction of transmembrane AMPA receptor regulatory proteins with multiple membrane associated guanylate kinases. Neuropharmacology 45:849856.

De Simoni A, Griesinger CB, Edwards FA (2003) Development of rat CA1 neurones in acute versus organotypic slices: role of experience in synaptic morphology and activity. The Journal of physiology 550:135-147.

Derkach V, Barria A, Soderling TR (1999) Ca2+/calmodulin-kinase II enhances channel conductance of alpha-amino-3-hydroxy-5-methyl-4isoxazolepropionate type glutamate receptors. Proceedings of the National Academy of Sciences of the United States of America 96:32693274.

Dev KK, Nishimune A, Henley JM, Nakanishi S (1999) The protein kinase C alpha binding protein PICK1 interacts with short but not long form alternative splice variants of AMPA receptor subunits. Neuropharmacology 38:635644.

Dingledine R, Borges K, Bowie D, Traynelis SF (1999) The glutamate receptor ion channels. Pharmacological reviews 51:7-61.

Doly HCBaJ (1979) A rapid alkaline extraction procedure for screening recombinant plasmid DNA. Nucleic Acids Res 7:1513-1523.

Ehlers MD (2000) Reinsertion or degradation of AMPA receptors determined by activity-dependent endocytic sorting. Neuron 28:511-525.

Ehrlich I (2004) Postsynaptic density 95 controls AMPA receptor incorporation during long-term potentiation and experience-driven synaptic plasticity. The Journal of neuroscience.

Ehrlich I, Klein M, Rumpel S, Malinow R (2007) PSD-95 is required for activitydriven synapse stabilization. Proceedings of the National Academy of Sciences of the United States of America 104:4176-4181.

El-Husseini AE, Craven SE, Chetkovich DM, Firestein BL, Schnell E, Aoki C, Bredt DS (2000a) Dual palmitoylation of PSD-95 mediates its vesiculotubular sorting, postsynaptic targeting, and ion channel clustering. The Journal of Cell Biology 148:159-172.

El-Husseini AE, Schnell E, Chetkovich DM, Nicoll RA, Bredt DS (2000b) PSD-95 involvement in maturation of excitatory synapses. Science 290:13641368.

El-Husseini AE, Topinka JR, Lehrer-Graiwer JE, Firestein BL, Craven SE, Aoki C, Bredt DS (2000c) Ion channel clustering by membrane-associated 
guanylate kinases. Differential regulation by N-terminal lipid and metal binding motifs. The Journal of biological chemistry 275:23904-23910.

Elias GM, Elias LAB, Apostolides PF, Kriegstein AR, Nicoll RA (2008) Differential trafficking of AMPA and NMDA receptors by SAP102 and PSD-95 underlies synapse development. Proceedings of the National Academy of Sciences of the United States of America 105:20953-20958.

Elias GM, Funke L, Stein V, Grant SG, Bredt DS, Nicoll RA (2006) Synapse-specific and developmentally regulated targeting of AMPA receptors by a family of MAGUK scaffolding proteins. Neuron 52:307-320.

Elias GM, Nicoll RA (2007) Synaptic trafficking of glutamate receptors by MAGUK scaffolding proteins. Trends in cell biology 17:343-352.

Feng W, Zhang M (2009) Organization and dynamics of PDZ-domain-related supramodules in the postsynaptic density. Nature Reviews Neuroscience 10:87-99.

Fukaya M, Tsujita M, Yamazaki M, Kushiya E, Abe M, Akashi K, Natsume R, Kano M, Kamiya H, Watanabe M, Sakimura K (2006) Abundant distribution of TARP gamma-8 in synaptic and extrasynaptic surface of hippocampal neurons and its major role in AMPA receptor expression on spines and dendrites. European Journal of Neuroscience 24:2177-2190.

Funke L, Dakoji S, Bredt DS (2005) Membrane-associated guanylate kinases regulate adhesion and plasticity at cell junctions. Annual Review of Biochemistry 74:219-245.

Futai K, Kim MJ, Hashikawa T, Scheiffele P, Sheng M, Hayashi Y (2007) Retrograde modulation of presynaptic release probability through signaling mediated by PSD-95-neuroligin. Nature Neuroscience 10:186195.

G ahwiler (1981) Organotypic monolayer cultures of nervous tissue. J Neurosci Methods 4:329-342.

Gallo V, Upson LM, Hayes WP, Vyklicky L, Winters CA, Buonanno A (1992) Molecular cloning and development analysis of a new glutamate receptor subunit isoform in cerebellum. The Journal of neuroscience : the official journal of the Society for Neuroscience 12:1010-1023.

Garner CC, Kindler S (1996) Synaptic proteins and the assembly of synaptic junctions. Trends in cell biology 6:429-433.

Greger IH, Khatri L, Ziff EB (2002) RNA editing at arg607 controls AMPA receptor exit from the endoplasmic reticulum. Neuron 34:759-772.

Groc L, Heine M, Cognet L, Brickley K, Stephenson FA, Lounis B, Choquet D (2004) Differential activity-dependent regulation of the lateral mobilities of AMPA and NMDA receptors. Nature Neuroscience 7:695-696.

Harris EW, Ganong AH, Cotman CW (1984) Long-term potentiation in the hippocampus involves activation of $\mathrm{N}$-methyl-D-aspartate receptors. Brain research 323:132-137.

Hashimoto K, Fukaya M, Qiao X, Sakimura K, Watanabe M, Kano M (1999) Impairment of AMPA receptor function in cerebellar granule cells of ataxic mutant mouse stargazer. Journal of Neuroscience 19:6027-6036. 
Hayashi MK, Tang C, Verpelli C, Narayanan R, Stearns MH, Xu R-M, Li H, Sala C, Hayashi Y (2009) The Postsynaptic Density Proteins Homer and Shank Form a Polymeric Network Structure. Cell 137:159-171.

Hayashi Y, Shi SH, Esteban JA, Piccini A, Poncer JC, Malinow R (2000) Driving AMPA receptors into synapses by LTP and CaMKII: requirement for GluR1 and PDZ domain interaction. Science 287:2262-2267.

Higuchi M, Single FN, Köhler M, Sommer B, Sprengel R, Seeburg PH (1993) RNA editing of AMPA receptor subunit GluR-B: a base-paired intron-exon structure determines position and efficiency. Cell 75:1361-1370.

Hirao K, Hata Y, Yao I, Deguchi M, Kawabe H, Mizoguchi A, Takai Y (2000) Three isoforms of synaptic scaffolding molecule and their characterization. Multimerization between the isoforms and their interaction with $\mathrm{N}$ methyl-D-aspartate receptors and SAP90/PSD-95-associated protein. The Journal of biological chemistry 275:2966-2972.

Hirokawa N, Takemura R (2005) Molecular motors and mechanisms of directional transport in neurons. Nature Reviews Neuroscience 6:201214.

Hollmann M, Heinemann S (1994) Cloned glutamate receptors. Annu Rev Neurosci 17:31-108.

Horton AC, Ehlers MD (2003) Neuronal polarity and trafficking. Neuron 40:277295.

Hsueh YP, Kim E, Sheng M (1997) Disulfide-linked head-to-head multimerization in the mechanism of ion channel clustering by PSD-95. Neuron 18:803814.

Hsueh YP, Sheng M (1999) Requirement of N-terminal cysteines of PSD-95 for PSD-95 multimerization and ternary complex formation, but not for binding to potassium channel Kv1.4. The Journal of biological chemistry 274:532-536.

Hsueh YP, Wang TF, Yang FC, Sheng M (2000) Nuclear translocation and transcription regulation by the membrane-associated guanylate kinase CASK/LIN-2. Nature 404:298-302.

Husi H, Grant SG (2001) Isolation of 2000-kDa complexes of N-methyl-Daspartate receptor and postsynaptic density 95 from mouse brain. Journal of Neurochemistry 77:281-291.

Husi H, Ward MA, Choudhary JS, Blackstock WP, Grant SG (2000) Proteomic analysis of NMDA receptor-adhesion protein signaling complexes. Nature Neuroscience 3:661-669.

Irie M, Hata Y, Takeuchi M, Ichtchenko K, Toyoda A, Hirao K, Takai Y, Rosahl TW, Südhof TC (1997) Binding of neuroligins to PSD-95. Science 277:15111515.

Isaac JTR, Ashby M, McBain CJ (2007) The role of the GluR2 subunit in AMPA receptor function and synaptic plasticity. Neuron 54:859-871.

Jackson AC, Nicoll RA (2011) The Expanding Social Network of Ionotropic Glutamate Receptors: TARPs and Other Transmembrane Auxiliary Subunits. Neuron 70:178-199. 
Jay SD, Ellis SB, McCue AF, Williams ME, Vedvick TS, Harpold MM, Campbell KP (1990) Primary structure of the gamma subunit of the DHP-sensitive calcium channel from skeletal muscle. Science 248:490-492.

Jo J, Son GH, Winters BL, Kim MJ, Whitcomb DJ, Dickinson BA, Lee Y-B, Futai K, Amici M, Sheng M, Collingridge GL, Cho K (2010) Muscarinic receptors induce LTD of NMDAR EPSCs via a mechanism involving hippocalcin, AP2 and PSD-95. Nature Neuroscience.

Jo K, Derin R, Li M, Bredt DS (1999) Characterization of MALS/Velis-1, -2, and -3: a family of mammalian LIN-7 homologs enriched at brain synapses in association with the postsynaptic density-95/NMDA receptor postsynaptic complex. Journal of Neuroscience 19:4189-4199.

Ju W, Morishita W, Tsui J, Gaietta G, Deerinck TJ, Adams SR, Garner CC, Tsien RY, Ellisman MH, Malenka RC (2004) Activity-dependent regulation of dendritic synthesis and trafficking of AMPA receptors. Nature Neuroscience 7:244-253.

K. S. Lundberg DDS, M. W. Adams, J. M. Short, J. A. Sorge, and E. J., Mathur. (1991) High-fidelity amplification using a thermostable DNA polymerase isolated from Pyrococcus furiosus. Gene 108:1-6.

Kato AS, Gill MB, Ho MT, Yu H, Tu Y, Siuda ER, Wang H, Qian Y-W, Nisenbaum ES, Tomita S, Bredt DS (2010a) Hippocampal AMPA Receptor Gating Controlled by\&amp;nbsp;Both TARP and Cornichon Proteins. Neuron 68:1082-1096.

Kato AS, Gill MB, Yu H, Nisenbaum ES, Bredt DS (2010b) TARPs differentially decorate AMPA receptors to specify neuropharmacology. Trends in neurosciences.

Kato AS, Zhou W, Milstein AD, Knierman MD, Siuda ER, Dotzlaf JE, Yu H, Hale JE, Nisenbaum ES, Nicoll RA, Bredt DS (2007) New transmembrane AMPA receptor regulatory protein isoform, gamma-7, differentially regulates AMPA receptors. Journal of Neuroscience 27:4969-4977.

Kawashima N, Sobue K (1997) Dijerential expression of isoforms of PSD-95 binding protein

(GKAP/SAPAP1) during rat brain development. FEBS letters 418:301-304.

Kennedy MB (2000) Signal-processing machines at the postsynaptic density. Science 290:750-754.

Kesner JLRaRP (Aug 2006) Lesions of the dorsal hippocampus or parietal cortex differentially affect spatial information processing. Behav Neurosci, 120:852-860.

Kesner RP, Hopkins RO (2006) Mnemonic functions of the hippocampus: a comparison between animals and humans. Biological psychology 73:3-18.

Kim E, Cho KO, Rothschild A, Sheng M (1996) Heteromultimerization and NMDA receptor-clustering activity of Chapsyn-110, a member of the PSD-95 family of proteins. Neuron 17:103-113.

Kim E, Naisbitt S, Hsueh YP, Rao A, Rothschild A, Craig AM, Sheng M (1997) GKAP, a novel synaptic protein that interacts with the guanylate kinaselike domain of the PSD-95/SAP90 family of channel clustering molecules. The Journal of Cell Biology 136:669-678. 
Kim E, Sheng M (2004) PDZ domain proteins of synapses. Nature Reviews Neuroscience 5:771-781.

Kim K, Yang J, Kim E (2010) Diacylglycerol kinases in the regulation of dendritic spines. Journal of Neurochemistry 112:577-587.

Kim MJ, Futai K, Jo J, Hayashi Y, Cho K, Sheng M (2007) Synaptic accumulation of PSD-95 and synaptic function regulated by phosphorylation of serine-295 of PSD-95. Neuron 56:488-502.

Kistner U, Wenzel BM, Veh RW, Cases-Langhoff C, Garner AM, Appeltauer U, Voss B, Gundelfinger ED, Garner CC (1993) SAP90, a rat presynaptic protein related to the product of the Drosophila tumor suppressor gene dlg-A. The Journal of biological chemistry 268:4580-4583.

Kleckner NW, Dingledine R (1988) Requirement for glycine in activation of NMDA-receptors expressed in Xenopus oocytes. Science 241:835-837.

Köhler M, Kornau HC, Seeburg PH (1994) The organization of the gene for the functionally dominant alpha-amino-3-hydroxy-5-methylisoxazole-4propionic acid receptor subunit GluR-B. The Journal of biological chemistry 269:17367-17370.

Kornau HC, Schenker LT, Kennedy MB, Seeburg PH (1995) Domain interaction between NMDA receptor subunits and the postsynaptic density protein PSD-95. Science 269:1737-1740.

Kristiansen LV, Beneyto M, Haroutunian V, Meador-Woodruff JH (2006) Changes in NMDA receptor subunits and interacting PSD proteins in dorsolateral prefrontal and anterior cingulate cortex indicate abnormal regional expression in schizophrenia. Mol Psychiatry 11:737-747.

Kuhlendahl S, Spangenberg O, Konrad M, Kim E, Garner CC (1998) Functional analysis of the guanylate kinase-like domain in the synapse-associated protein SAP97. European journal of biochemistry / FEBS 252:305-313.

Lee S, Fan S, Makarova O, Straight S, Margolis B (2002) A novel and conserved protein-protein interaction domain of mammalian Lin-2/CASK binds and recruits SAP97 to the lateral surface of epithelia. Molecular and cellular biology 22:1778-1791.

Li Y, Hu J, Höfer K, Wong AMS, Cooper JD, Birnbaum SG, Hammer RE, Hofmann SL (2010) DHHC5 interacts with PDZ domain 3 of post-synaptic density-95 (PSD-95) protein and plays a role in learning and memory. The Journal of biological chemistry.

Lin D-T, Makino Y, Sharma K, Hayashi T, Neve R, Takamiya K, Huganir RL (2009) Regulation of AMPA receptor extrasynaptic insertion by $4.1 \mathrm{~N}$, phosphorylation and palmitoylation. Nature Neuroscience 12:879-887.

Lomeli H, Mosbacher J, Melcher T, Höger T, Geiger JR, Kuner T, Monyer H, Higuchi M, Bach A, Seeburg PH (1994) Control of kinetic properties of AMPA receptor channels by nuclear RNA editing. Science 266:1709-1713.

Lu W, Man H, Ju W, Trimble WS, MacDonald JF, Wang YT (2001) Activation of synaptic NMDA receptors induces membrane insertion of new AMPA receptors and LTP in cultured hippocampal neurons. Neuron 29:243-254.

Lue RA, Marfatia SM, Branton D, Chishti AH (1994) Cloning and characterization of hdlg: the human homologue of the Drosophila discs large tumor 
suppressor binds to protein 4.1. Proceedings of the National Academy of Sciences of the United States of America 91:9818-9822.

Magleby KL (2004) Modal gating of NMDA receptors. Trends in neurosciences 27:231-233.

Malenka RC, Nicoll RA (1999) Long-term potentiation--a decade of progress? Science 285:1870-1874.

Malinow R, Malenka RC (2002) AMPA receptor trafficking and synaptic plasticity. Annu Rev Neurosci 25:103-126.

Mammen AL, Huganir RL, O\&apos;Brien RJ (1997) Redistribution and stabilization of cell surface glutamate receptors during synapse formation. The Journal of neuroscience : the official journal of the Society for Neuroscience 17:7351-7358.

Mano I, Teichberg VI (1998) A tetrameric subunit stoichiometry for a glutamate receptor-channel complex. Neuroreport 9:327-331.

Mansour M, Nagarajan N, Nehring RB, Clements JD, Rosenmund C (2001) Heteromeric AMPA receptors assemble with a preferred subunit stoichiometry and spatial arrangement. Neuron 32:841-853.

Marfatia SM, Byron O, Campbell G, Liu SC, Chishti AH (2000) Human homologue of the Drosophila discs large tumor suppressor protein forms an oligomer in solution. Identification of the self-association site. The Journal of biological chemistry 275:13759-13770.

Marfatia SM, Morais-Cabral JH, Kim AC, Byron O, Chishti AH (1997) The PDZ domain of human erythrocyte p55 mediates its binding to the cytoplasmic carboxyl terminus of glycophorin C. Analysis of the binding interface by in vitro mutagenesis. The Journal of biological chemistry 272:24191-24197.

Martin SJ, Grimwood PD, Morris RG (2000) Synaptic plasticity and memory: an evaluation of the hypothesis. Annu Rev Neurosci 23:649-711.

McGee AW, Bredt DS (1999) Identification of an intramolecular interaction between the SH3 and guanylate kinase domains of PSD-95. The Journal of biological chemistry 274:17431-17436.

McGee AW, Dakoji SR, Olsen O, Bredt DS, Lim WA, Prehoda KE (2001) Structure of the SH3-guanylate kinase module from PSD-95 suggests a mechanism for regulated assembly of MAGUK scaffolding proteins. Molecular cell 8:1291-1301.

Migaud M, Charlesworth P, Dempster M, Webster LC, Watabe AM, Makhinson M, He Y, Ramsay MF, Morris RG, Morrison JH, O\&apos;dell TJ, Grant SG (1998) Enhanced long-term potentiation and impaired learning in mice with mutant postsynaptic density-95 protein. Nature 396:433-439.

Milstein AD, Zhou W, Karimzadegan S, Bredt DS, Nicoll RA (2007) TARP subtypes differentially and dose-dependently control synaptic AMPA receptor gating. Neuron 55:905-918.

Montgomery JM, Zamorano PL, Garner CC (2004) MAGUKs in synapse assembly and function: an emerging view. Cellular and molecular life sciences : CMLS 61:911-929.

Moss FJ, Dolphin AC, Clare JJ (2003) Human neuronal stargazin-like proteins, gamma2, gamma3 and gamma4; an investigation of their specific localization in human brain and their influence on CaV2.1 voltage- 
dependent calcium channels expressed in Xenopus oocytes. BMC neuroscience 4:23.

Mothet JP, Parent AT, Wolosker H, Brady RO, Linden DJ, Ferris CD, Rogawski MA, Snyder SH (2000) D-serine is an endogenous ligand for the glycine site of the N-methyl-D-aspartate receptor. Proceedings of the National Academy of Sciences of the United States of America 97:4926-4931.

Müller BM, Kistner U, Kindler S, Chung WJ, Kuhlendahl S, Fenster SD, Lau LF, Veh RW, Huganir RL, Gundelfinger ED, Garner CC (1996) SAP102, a novel postsynaptic protein that interacts with NMDA receptor complexes in vivo. Neuron 17:255-265.

Müller BM, Kistner U, Veh RW, Cases-Langhoff C, Becker B, Gundelfinger ED, Garner CC (1995) Molecular characterization and spatial distribution of SAP97, a novel presynaptic protein homologous to SAP90 and the Drosophila discs-large tumor suppressor protein. The Journal of neuroscience : the official journal of the Society for Neuroscience 15:2354-2366.

Murata Y, Doi T, Taniguchi H, Fujiyoshi Y (2005) Proteomic analysis revealed a novel synaptic proline-rich membrane protein (PRR7) associated with PSD-95 and NMDA receptor. Biochemical and biophysical research communications 327:183-191.

Naisbitt S, Kim E, Tu JC, Xiao B, Sala C, Valtschanoff J, Weinberg RJ, Worley PF, Sheng M (1999) Shank, a Novel Family of Postsynaptic Density Proteins that Binds to the NMDA Receptor/PSD-95/GKAP Complex and Cortactin. Neuron 23:569-582.

Nakagawa T, Futai K, Lashuel HA, Lo I, Okamoto K, Walz T, Hayashi Y, Sheng M (2004) Quaternary structure, protein dynamics, and synaptic function of SAP97 controlled by L27 domain interactions. Neuron 44:453-467.

Neves G, Cooke SF, Bliss TVP (2008) Synaptic plasticity, memory and the hippocampus: a neural network approach to causality. Nature Reviews Neuroscience 9:65-75.

Newman RA, Prehoda KE (2009) Intramolecular interactions between the SRC homology 3 and guanylate kinase domains of discs large regulate its function in asymmetric cell division. The Journal of biological chemistry 284:12924-12932.

Nicoll RA, Tomita S, Bredt DS (2006) Auxiliary subunits assist AMPA-type glutamate receptors. Science 311:1253-1256.

Niethammer M, Kim E, Sheng M (1996) Interaction between the C terminus of NMDA receptor subunits and multiple members of the PSD-95 family of membrane-associated guanylate kinases. The Journal of neuroscience : the official journal of the Society for Neuroscience 16:2157-2163.

Nix SL, Chishti AH, Anderson JM, Walther Z (2000) hCASK and hDlg associate in epithelia, and their src homology 3 and guanylate kinase domains participate in both intramolecular and intermolecular interactions. The Journal of biological chemistry 275:41192-41200.

Noebels JL, Qiao X, Bronson RT, Spencer C, Davisson MT (1990) Stargazer: a new neurological mutant on chromosome 15 in the mouse with prolonged cortical seizures. Epilepsy research 7:129-135. 
Novak L BP, P Asher (1984) Magnesium gates glutamate activated channels in mouse central neurones. Nature 84.

Olsen 0, Bredt DS (2003) Functional analysis of the nucleotide binding domain of membrane-associated guanylate kinases. The Journal of biological chemistry 278:6873-6878.

Osten P, Stern-Bach Y (2006) Learning from stargazin: the mouse, the phenotype and the unexpected. Current opinion in neurobiology 16:275-280.

P. Andersen RM, D. Amaral, T. Bliss, and J. O'Keefe, editors (2007) The Hippocampus Book (Oxford Neuroscience Series). Oxford University Press, USA, 1 edition, 112007 ISBN 9780195100273.

Pak DT, Yang S, Rudolph-Correia S, Kim E, Sheng M (2001) Regulation of dendritic spine morphology by SPAR, a PSD-95-associated RapGAP. Neuron 31:289-303.

Palmer CL, Cotton L, Henley JM (2005) The molecular pharmacology and cell biology of alpha-amino-3-hydroxy-5-methyl-4-isoxazolepropionic acid receptors. Pharmacological reviews 57:253-277.

Panatier A, Oliet SHR (2006) Neuron-glia interactions in the hypothalamus. Neuron glia biology 2:51-58.

Park M, Salgado JM, Ostroff L, Helton TD, Robinson CG, Harris KM, Ehlers MD (2006) Plasticity-induced growth of dendritic spines by exocytic trafficking from recycling endosomes. Neuron 52:817-830.

Parker MJ, Zhao S, Bredt DS, Sanes JR, Feng G (2004) PSD93 regulates synaptic stability at neuronal cholinergic synapses. Journal of Neuroscience 24:378-388.

Passafaro M, Piëch V, Sheng M (2001) Subunit-specific temporal and spatial patterns of AMPA receptor exocytosis in hippocampal neurons. Nature Neuroscience 4:917-926.

Penfield. BMaW (1955) The effect of hippocampal lesions on recent memory. Trans Am Neurol Assoc, (80th Meeting) 42\{48.

Petersen JD, Chen X, Vinade L, Dosemeci A, Lisman JE, Reese TS (2003) Distribution of postsynaptic density (PSD)-95 and Ca2+/calmodulindependent protein kinase II at the PSD. Journal of Neuroscience 23:11270-11278.

Petralia RS, Wang Y-X, Wenthold RJ (2003) Internalization at glutamatergic synapses during development. European Journal of Neuroscience 18:3207-3217.

Pierce JP, Mayer T, McCarthy JB (2001) Evidence for a satellite secretory pathway in neuronal dendritic spines. Current Biology 11:351-355.

R. K. Saiki DHG, S. Stoffel, S. J. Scharf, R. Higuchi, G. T. Horn, K. B. Mullis, and H. A. Erlich. (1988) Primer-directed enzymatic amplification of DNA with a thermostable DNA polymerase. Science 239:487-491.

Reese ML, Dakoji S, Bredt DS, Dötsch V (2007) The guanylate kinase domain of the MAGUK PSD-95 binds dynamically to a conserved motif in MAP1a. Nature Structural \&amp;\#38; Molecular Biology 14:155-163.

Roche KW, O\&apos;Brien RJ, Mammen AL, Bernhardt J, Huganir RL (1996) Characterization of multiple phosphorylation sites on the AMPA receptor GluR1 subunit. Neuron 16:1179-1188. 
Rogers SW, Hughes TE, Hollmann M, Gasic GP, Deneris ES, Heinemann S (1991) The characterization and localization of the glutamate receptor subunit GluR1 in the rat brain. The Journal of neuroscience : the official journal of the Society for Neuroscience 11:2713-2724.

Rosenmund C, Stern-Bach Y, Stevens CF (1998) The tetrameric structure of a glutamate receptor channel. Science 280:1596-1599.

Rumbaugh G, Sia G-M, Garner CC, Huganir RL (2003) Synapse-associated protein-97 isoform-specific regulation of surface AMPA receptors and synaptic function in cultured neurons. Journal of Neuroscience 23:45674576.

Sambrook J (2001) Molecular Cloning: A Laboratory Manual, Third Edition (3 Volume Set). Cold Spring Harbor Laboratory Press ISBN 0879695773.

Sans N, Petralia RS, Wang YX, Blahos J, Hell JW, Wenthold RJ (2000) A developmental change in NMDA receptor-associated proteins at hippocampal synapses. Journal of Neuroscience 20:1260-1271.

Sans N, Racca C, Petralia RS, Wang YX, McCallum J, Wenthold RJ (2001) Synapseassociated protein 97 selectively associates with a subset of AMPA receptors early in their biosynthetic pathway. Journal of Neuroscience 21:7506-7516.

Sans N, Vissel B, Petralia RS, Wang Y-X, Chang K, Royle GA, Wang C-Y, O\&apos;Gorman S, Heinemann SF, Wenthold RJ (2003) Aberrant formation of glutamate receptor complexes in hippocampal neurons of mice lacking the GluR2 AMPA receptor subunit. Journal of Neuroscience 23:9367-9373.

Scannevin RH, Huganir RL (2000) Postsynaptic organization and regulation of excitatory synapses. Nature Reviews Neuroscience 1:133-141.

Schlüter OM, Xu W, Malenka RC (2006) Alternative N-terminal domains of PSD95 and SAP97 govern activity-dependent regulation of synaptic AMPA receptor function. Neuron 51:99-111.

Schnell E, Sizemore M, Karimzadegan S, Chen L, Bredt DS, Nicoll RA (2002) Direct interactions between PSD-95 and stargazin control synaptic AMPA receptor number. Proceedings of the National Academy of Sciences of the United States of America 99:13902-13907.

Schwenk J, Harmel N, Zolles G, Bildl W, Kulik A, Heimrich B, Chisaka O, Jonas P, Schulte U, Fakler B, Klocker N (2009) Functional Proteomics Identify Cornichon Proteins as Auxiliary Subunits of AMPA Receptors. Science 323:1313-1319.

Seabold GK (2003) Interaction of the Tyrosine Kinase Pyk2 with the N-Methyl-Daspartate Receptor Complex via the Src Homology 3 Domains of PSD-95 and SAP102. Journal of Biological Chemistry 278:15040-15048.

Setou M, Seog D-H, Tanaka Y, Kanai Y, Takei Y, Kawagishi M, Hirokawa N (2002) Glutamate-receptor-interacting protein GRIP1 directly steers kinesin to dendrites. Nature 417:83-87.

Sheng M, Kim MJ (2002) Postsynaptic signaling and plasticity mechanisms. Science 298:776-780.

Sheng M, Sala C (2001) PDZ domains and the organization of supramolecular complexes. Annu Rev Neurosci 24:1-29. 
Shi S, Hayashi Y, Esteban JA, Malinow R (2001) Subunit-specific rules governing AMPA receptor trafficking to synapses in hippocampal pyramidal neurons. Cell 105:331-343.

Shi Y, Lu W, Milstein AD, Nicoll RA (2009) The stoichiometry of AMPA receptors and TARPs varies by neuronal cell type. Neuron 62:633-640.

Shin H, Hsueh YP, Yang FC, Kim E, Sheng M (2000) An intramolecular interaction between Src homology 3 domain and guanylate kinase-like domain required for channel clustering by postsynaptic density-95/SAP90. Journal of Neuroscience 20:3580-3587.

Shipman SL, Schnell E, Hirai T, Chen B-S, Roche KW, Nicoll RA (2011) Functional dependence of neuroligin on a new non-PDZ intracellular domain. Nature Neuroscience 1-11.

Simeone TA, Sanchez RM, Rho JM (2004) Molecular biology and ontogeny of glutamate receptors in the mammalian central nervous system. Journal of child neurology 19:343-360; discussion 361.

Sommer B, Keinänen K, Verdoorn TA, Wisden W, Burnashev N, Herb A, Köhler M, Takagi T, Sakmann B, Seeburg PH (1990) Flip and flop: a cell-specific functional switch in glutamate-operated channels of the CNS. Science 249:1580-1585.

Sommer B, Köhler M, Sprengel R, Seeburg PH (1991) RNA editing in brain controls a determinant of ion flow in glutamate-gated channels. Cell 67:11-19.

Soto D, Coombs ID, Kelly L, Farrant M, Cull-Candy SG (2007) Stargazin attenuates intracellular polyamine block of calcium-permeable AMPA receptors. Nature Neuroscience 10:1260-1267.

Soto D, Coombs ID, Renzi M, Zonouzi M, Farrant M, Cull-Candy SG (2009) Selective regulation of long-form calcium-permeable AMPA receptors by an atypical TARP, gamma-5. Nature Neuroscience 12:277-285.

Stein V, House DRC, Bredt DS, Nicoll RA (2003) Postsynaptic density-95 mimics and occludes hippocampal long-term potentiation and enhances longterm depression. Journal of Neuroscience 23:5503-5506.

Stoppini L, Buchs PA, Muller D (1991) A simple method for organotypic cultures of nervous tissue. Journal of neuroscience methods 37:173-182.

Sturgill JF, Steiner P, Czervionke BL, Sabatini BL (2009) Distinct domains within PSD-95 mediate synaptic incorporation, stabilization, and activitydependent trafficking. Journal of Neuroscience 29:12845-12854.

Sutton MA, Ito HT, Cressy P, Kempf C, Woo JC, Schuman EM (2006) Miniature neurotransmission stabilizes synaptic function via tonic suppression of local dendritic protein synthesis. Cell 125:785-799.

Sweet ES, Previtera ML, Fernández JR, Charych EI, Tseng C-Y, Kwon M, Starovoytov V, Zheng JQ, Firestein BL (2011) PSD-95 Alters Microtubule Dynamics via an Association With EB3. The Journal of neuroscience : the official journal of the Society for Neuroscience 31:1038-1047.

Takeuchi M, Hata Y, Hirao K, Toyoda A, Irie M, Takai Y (1997) SAPAPs. A family of PSD-95/SAP90-associated proteins localized at postsynaptic density. The Journal of biological chemistry 272:11943-11951. 
Tardin C, Cognet L, Bats C, Lounis B, Choquet D (2003) Direct imaging of lateral movements of AMPA receptors inside synapses. The EMBO Journal 22:4656-4665.

Tavares GA, Panepucci EH, Brunger AT (2001) Structural characterization of the intramolecular interaction between the $\mathrm{SH} 3$ and guanylate kinase domains of PSD-95. Molecular cell 8:1313-1325.

Tomita S, Adesnik H, Sekiguchi M, Zhang W, Wada K, Howe JR, Nicoll RA, Bredt DS (2005a) Stargazin modulates AMPA receptor gating and trafficking by distinct domains. Nature 435:1052-1058.

Tomita S, Chen L, Kawasaki Y, Petralia RS, Wenthold RJ, Nicoll RA, Bredt DS (2003) Functional studies and distribution define a family of transmembrane AMPA receptor regulatory proteins. The Journal of Cell Biology 161:805-816.

Tomita S, Fukata M, Nicoll RA, Bredt DS (2004) Dynamic interaction of stargazinlike TARPs with cycling AMPA receptors at synapses. Science 303:15081511.

Tomita S, Stein V, Stocker TJ, Nicoll RA, Bredt DS (2005b) Bidirectional synaptic plasticity regulated by phosphorylation of stargazin-like TARPs. Neuron 45:269-277.

Topinka JR, Bredt DS (1998) N-terminal palmitoylation of PSD-95 regulates association with cell membranes and interaction with $\mathrm{K}+$ channel Kv1.4. Neuron 20:125-134.

Towbin H, Staehelin T, Gordon J (1979) Electrophoretic transfer of proteins from polyacrylamide gels to nitrocellulose sheets: procedure and some applications. Proceedings of the National Academy of Sciences of the United States of America 76:4350-4354.

Triller A, Choquet D (2005) Surface trafficking of receptors between synaptic and extrasynaptic membranes: and yet they do move! Trends in neurosciences 28:133-139.

Valtschanoff JG, Weinberg RJ (2001) Laminar organization of the NMDA receptor complex within the postsynaptic density. Journal of Neuroscience 21:1211-1217.

Vandenberghe W, Bredt DS (2004) Early events in glutamate receptor trafficking. Current opinion in cell biology 16:134-139.

Vandenberghe W, Nicoll RA, Bredt DS (2005) Interaction with the unfolded protein response reveals a role for stargazin in biosynthetic AMPA receptor transport. Journal of Neuroscience 25:1095-1102.

Verdoorn TA, Burnashev N, Monyer H, Seeburg PH, Sakmann B (1991) Structural determinants of ion flow through recombinant glutamate receptor channels. Science 252:1715-1718.

von Engelhardt J, Mack V, Sprengel R, Kavenstock N, Li KW, Stern-Bach Y, Smit AB, Seeburg PH, Monyer H (2010) CKAMP44: a brain-specific protein attenuating short-term synaptic plasticity in the dentate gyrus. Science 327:1518-1522.

Waites CL, Specht CG, Härtel K, Leal-Ortiz S, Genoux D, Li D, Drisdel RC, Jeyifous O, Cheyne JE, Green WN, Montgomery JM, Garner CC (2009) Synaptic 
SAP97 isoforms regulate AMPA receptor dynamics and access to presynaptic glutamate. Journal of Neuroscience 29:4332-4345.

Walikonis RS, Jensen ON, Mann M, Provance DW, Mercer JA, Kennedy MB (2000) Identification of proteins in the postsynaptic density fraction by mass spectrometry. Journal of Neuroscience 20:4069-4080.

Wang JQ, Arora A, Yang L, Parelkar NK, Zhang G, Liu X, Choe ES, Mao L (2005) Phosphorylation of AMPA receptors: mechanisms and synaptic plasticity. Molecular neurobiology 32:237-249.

Watkins JC, Evans RH (1981) Excitatory Amino Acid Transmitters. Annual Review of Pharmacology and Toxicology 21:165-204.

Wenthold RJ, Petralia RS, Blahos J II, Niedzielski AS (1996) Evidence for multiple AMPA receptor complexes in hippocampal CA1/CA2 neurons. The Journal of neuroscience : the official journal of the Society for Neuroscience 16:1982-1989.

Wenthold RJ, Prybylowski K, Standley S, Sans N, Petralia RS (2003) Trafficking of NMDA receptors. Annual review of pharmacology and toxicology 43:335358.

Wu H, Nash JE, Zamorano P, Garner CC (2002) Interaction of SAP97 with minusend-directed actin motor myosin VI. Implications for AMPA receptor trafficking. The Journal of biological chemistry 277:30928-30934.

Wyszynski M, Valtschanoff JG, Naisbitt S, Dunah AW, Kim E, Standaert DG, Weinberg R, Sheng M (1999) Association of AMPA receptors with a subset of glutamate receptor-interacting protein in vivo. The Journal of neuroscience : the official journal of the Society for Neuroscience 19:6528-6537.

Xu W, Schlüter OM, Steiner P, Czervionke BL, Sabatini B, Malenka RC (2008) Molecular dissociation of the role of PSD-95 in regulating synaptic strength and LTD. Neuron 57:248-262.

Yamauchi T (2002) Molecular constituents and phosphorylation-dependent regulation of the post-synaptic density. Mass spectrometry reviews 21:266-286.

Yao W-D, Gainetdinov RR, Arbuckle MI, Sotnikova TD, Cyr M, Beaulieu J-M, Torres GE, Grant SGN, Caron MG (2004) Identification of PSD-95 as a regulator of dopamine-mediated synaptic and behavioral plasticity. Neuron 41:625-638.

Yudowski GA, Puthenveedu MA, Leonoudakis D, Panicker S, Thorn KS, Beattie EC, von Zastrow M (2007) Real-time imaging of discrete exocytic events mediating surface delivery of AMPA receptors. Journal of Neuroscience 27:11112-11121.

Zheng C-Y, Petralia RS, Wang Y-X, Kachar B, Wenthold RJ (2010) SAP102 is a highly mobile MAGUK in spines. Journal of Neuroscience 30:4757-4766.

Ziff EB (2007) TARPs and the AMPA receptor trafficking paradox. Neuron 53:627-633. 


\section{Curriculum Vitae}

\section{Curriculum Vitae}

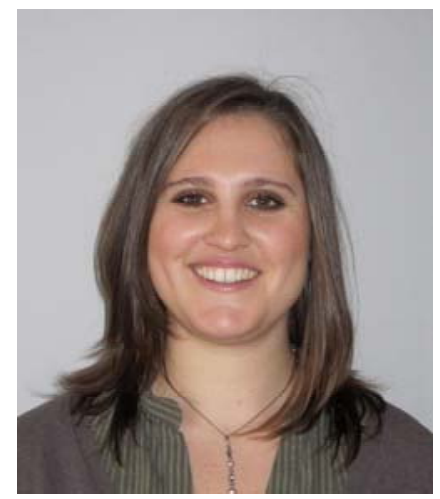

\section{Personal data}

Name:

Home address:

Cell-Phone:

Home-phone:

Date of birth:

Place of birth:

E-mail:

Nationality:
Stéphanie A.D. BONNET

47 bd de l'Ocean

13009 Marseille

France

+4917664614631

+33491717269

$6^{\text {th }}$ of August 1983

Marseilles (France)

stephanie.bonnet13@gmail.com

s.bonnet@eni-g.de

French 
Sex:

Female

Marital status: $\quad$ Single

\section{Academic Quallifications}

Sciences Faculty of Luminy, Mediterranean University Aix-Marseilles II.

2006

Master's degree in Biology of Eukaryotes (second year) specialization in neurobiology

2005

Master's degree in Biology of Eukaryotes (first year) specialization in neurobiology

2004

three-year university degree (LICENCE) in Cellular Biology

2003

two-year university degree (DEUG) in Life sciences

2001

Scientific Baccalauréat, specialization in Biology

\section{Laboratory training}

Sept. 2007 - Sept.2011 PhD student at the European Neuroscience InstituteGöttingen (ENI-G) under the supervision of Dr. Dr. O.Schlüter.

Role of the different domains of PSD-95 in basal synaptic transmission (molecular cloning, viral use, electrophysiology: visual patch-clamp, Biochemistry).

Oct. 2006 - June 2007

Work at the Zentrum fur Molekular Neurobiologie Hamburg (ZMNH), Institut für Biosynthese neuraler Strukturen (director: Prof. M.Schachner, Hamburg, Germany) under the supervision of the Dr.

A.Dityatev.

Modulation of neuronal activity by recognition molecule-associated polyanionic carbohydrates (electrophysiology: field recording)

January - June 2006

Training course of Master 2, at the Mediterranean Institute of Neurobiology (INMED), INSERM U29 (director: Prof. Y.Ben-Ari; Marseilles, France) under the supervision of the Dr. JL.GAIARSA.

Role of BDNF in the induction of GABAergic synaptic plasticity by spontaneous network driven activity in 
the neonatal rat hippocampus (electrophysiology: blind patch-clamp).

Sept.2005 - Jan.2006 Training course of Master 2, at the Mediterranean Institute of Neurobiology (INMED), INSERM U29 (director: Prof. Y. Ben-Ari; Marseilles, France) under the supervision of the Dr. S.KRANTIC.

Neuronal death induced by NMDA and link with the reinitiation of the cell cycle: the effect of NMDA on the induction of cyclin D1 as a function of synaptic- or extra-synaptic receptor stimulation in embryonic rat hippocampus (western blot, immunofluorescence).

February-April 2005 Training course of Master 1, at Developmental Biology Institute of Marseille (IBDM) (director: Dr. G.Rougon; Marseilles, France) in the department of axonal plasticity in the development and pathology INSERM UMR 623 under the supervision of Dr. C.HENDERSON.

Implication of Semaphorin in the establishment of pool-specific motoneurons within the spinal cord (in situ hybridization on whole mount spinal cord).

\section{Technical skills}

- Electrophysiology: visual patch clamp on organotypic hippocampal slices, blind patch clamp and field recordings on acute hippocampal slices

- Molecular cloning

- Virus injection

- Acute and organotypic slices preparation

- Biochemistry: Western-blot, immunocytochemistry, in situ hybridization

\section{Workshops participation}

- Team work \& leadership competencies by Dr. Simon Golin

- Effective leadership \& successful negotiations by Elisabeth Schick

- Time management by Dr. Simon Golin

- Scientific communication by Dr. Heather Silyn Roberts

- Male female communication by Marion Knaths

- Recognizing \& managing conflicts by Dr. Eva Himmelsbach

- Grant writing by Dr. Christina Schütte

- Career planning by Dr. Simon Golin

- Job-hunting by Dr. Ralf Pétri 


\section{Referees}

- Dr. Dr. Oliver Schlüter

oschlue@gwdg.de ; Tel. (+49) 5513910374

European Neuroscience Institute Göttingen (ENI-G), Germany

\section{- Dr. Jean-Luc GAIARSA}

gaiarsa@inmed.univ-mrs.fr ; Tel. (+33) 491828140

Institut de Neurobiologie de la Méditerranée (INMED), INSERM U29 (director:

Prof. Y. Ben-Ari; Marseilles, France).

- Dr. Slavica KRANTIC

slavica.krantic@douglas.mcgill.ca

krantic@inmed.univ-mrs.fr ; Tel. (+33) 491828138

Institut de Neurobiologie de la Méditerranée (INMED), INSERM U29 (director:

Prof. Y. Ben-Ari; Maseilles, France).

\section{Languages}

French : fluent (native)

English : fluent

German : basic (elementary knowledge)

\section{Interests}

Travelling, swimming, sailing, reading, theater, cinema. 
